\title{
The impact of hypoxemia and/or hypotension on electrical brain activity as measured using the Maastricht cerebral monitor
}

Citation for published version (APA):

Gavilanes Jimenez, A. W. D. (2003). The impact of hypoxemia and/or hypotension on electrical brain activity as measured using the Maastricht cerebral monitor. [Doctoral Thesis, Maastricht University]. Universiteit Maastricht. https://doi.org/10.26481/dis.20030320ag

Document status and date:

Published: 01/01/2003

DOI:

10.26481/dis.20030320ag

Document Version:

Publisher's PDF, also known as Version of record

Please check the document version of this publication:

- A submitted manuscript is the version of the article upon submission and before peer-review. There can be important differences between the submitted version and the official published version of record.

People interested in the research are advised to contact the author for the final version of the publication, or visit the DOI to the publisher's website.

- The final author version and the galley proof are versions of the publication after peer review.

- The final published version features the final layout of the paper including the volume, issue and page numbers.

Link to publication

\footnotetext{
General rights rights.

- You may freely distribute the URL identifying the publication in the public portal. please follow below link for the End User Agreement:

www.umlib.nl/taverne-license

Take down policy

If you believe that this document breaches copyright please contact us at:

repository@maastrichtuniversity.nl

providing details and we will investigate your claim.
}

Copyright and moral rights for the publications made accessible in the public portal are retained by the authors and/or other copyright owners and it is a condition of accessing publications that users recognise and abide by the legal requirements associated with these

- Users may download and print one copy of any publication from the public portal for the purpose of private study or research.

- You may not further distribute the material or use it for any profit-making activity or commercial gain

If the publication is distributed under the terms of Article $25 \mathrm{fa}$ of the Dutch Copyright Act, indicated by the "Taverne" license above, 


\section{THE IMPACT OF HYPOXEMIA AND/OR HYPOTENSION ON ELECTRICAL BRAIN ACTIVITY AS MEASURED USING THE MAASTRICHT CEREBRAL MONITOR}

(with conclusions and summary in english, dutch and spanish)

ANTONIO WENCESLAO DANILO GAVILANES JIMENEZ 


\title{
THE IMPACT OF HYPOXEMIA AND/OR HYPOTENSION ON ELECTRICAL BRAIN ACTIVITY AS MEASURED USING THE MAASTRICHT CEREBRAL MONITOR
}

\author{
PROEFSCHRIFT \\ ter verkrijging van de graad van doctor aan de \\ Universiteit Maastricht, \\ op gezag van de Rector Magnificus \\ Prof. dr. A.C. Nieuwenhuijzen Kruseman, \\ volgens het besluit van het College van Decanen, \\ in het openbaar te verdedigen op \\ donderdag 20 maart 2003 om 16:00 uur
}

door

ANTONIO WENCESLAO DANILO GAVILANES JIMENEZ

Geboren op 29 April 1960

te Guayaquil - Ecuador 


\section{Promotores}

Prof. dr. C.E. Blanco

Prof. dr. J.S.H. Vles

Co-promotor

Dr. ir. J.P.H. Reulen

\section{Beoordelingscommissie}

Prof. dr. J. Troost (voorzitter)

Prof. dr. P. Casaer (Universiteit van Leuven, België)

Prof. dr. J. Jolles

Prof. dr. H. Steinbusch

Prof. dr. L. de Vries (Universiteit van Utrecht)

ISBN 90-5681-151-7

The studies described in this thesis were performed at the research Institute Growth and Development (GROW), Maastricht University, The Netherlands.

The publication of this thesis was supported by (in alphabetical order) Abbott BV, Boehringer Ingelheim BV, Christiaens BV, Ferring BV and Nutricia Nederland BV. 
TO YAZMIN SALCEDO SEGURA

my inspiration

TO DANIELA, DIANA, DANILO ANDRES, EDUARDO AND JAVIER my life

TO MY PARENTS

who taught me to care 


\section{CONTENTS}

\section{Chapter 1}

Introduction and aims of the study

\section{Chapter 2}

Review of the literature

\section{Chapter 3}

The Maastricht Cerebral Monitor (MCM) for the neonatal intensive care unit.

Journal of Medical Engineering and Technology 1999; 23:1:29-37.

\section{Chapter 4}

The effect of repetitive umbilical cord occlusions on neuronal brain activity measured by the Cerebral Function Analysing Monitor and histological outcome in the immature fetal sheep.

J Soc Gynecol Investig 2000; 7(4): 218-23.

\section{Chapter 5}

The influence of acute hypoxemia and hypovolemic hypotension on neuronal brain activity measured by the Cerebral Function Monitor in newborn piglets. Neuropediatrics 1996; 27: 260-264.

\section{Chapter 6}

Electrocortical brain activity, cerebral haemodynamics and oxygenation during progressive hypotension.

Clin Neurophysiology 2001; 112(1): 52-59.

\section{Chapter 7}

Neonatal electrocortical brain activity and cerebral oxygenation during nonacidotic, normocarbic and normotensive graded hypoxemia.

Clin Neurophysiology, in press

\section{Chapter 8}

Electrical brain activity during normotensive hypoxemia in very low birth weight infants.

Neuropediatrics, accepted

\section{Chapter 9}

General discussion and summary

Algemene discussie en samenvatting

Discusión general y resumen

Acknowledgment and curriculum vitae 
CHAPTER 1.

INTRODUCTION AND AIMS OF THE STUDY

1.1. General considerations

1.2. Aims of the study

1.3. Summary 


\section{Introduction}

\subsection{General considerations}

The mortality of very low birth weight infants (VLBWI, birth weight less than $1500 \mathrm{~g}$ ) has fallen in the last 2 decades because of both the improvement of pre- and perinatal care and the regionalization of care. Pharmacological therapies have contributed significantly to this improvement by: 1) the use of surfactant in infant's suffering from respiratory distress syndrome (Guyer et al., 2000); and 2) the use of antenatal steroids (Hack et al., 2000). Nevertheless, there is still a high incidence of both mild and severe neurological abnormalities in neonatal intensive care unit (NICU) graduates (Vohr et al., 2000). In addition, no specific therapy has been proven to improve neurodevelopmental outcome. Postnatal indomethacin for instance, which reduces the incidence of major of periventricular-intraventricular hemorrhage $(\mathrm{PIVH})$ in tiny prematures, does not improve outcome at 18 months corrected age (Schmidt et al., 2001).

The long-term primary aim of neonatal care is an intact neurological outcome (Hack et al., 2000). However, on-line continuous neonatal monitoring concentrates exclusively on cardio-respiratory variables, while cerebral function is not taken into account. In this context, electrical brain activity (EBA) as depicted by an electroencephalogram (EEG) has been one of the most studied features of cerebral function. However, EEG recording takes place on a serial basis, if clinically indicated and not as continuous EBA monitoring in newborn infants.

Continuous EEG evaluation has been introduced in the NICU using the Cerebral Function Monitor (CFM), a one-channel continuous amplitudeintegrated EEG (aEEG). It was first described by Maynard et al. in 1969 in adult intensive care and 10 years later, in perinatal care (Maynard et al., 1969a; Maynard et al., 1979a and 1979b). Currently, the use of aEEG in the NICU can be seen as an on-line monitor of EBA patterns with prognostic value for term infants, as well as valuable in monitoring for the presence of seizure activity. To the contrary, scarce data has been published upon its use in preterm infants. The limited feasibility of neurological assessment in those infants, who are usually under the influence of sedative drugs, makes its use relevant in the NICU. Major difficulties in the interpretation of EEG in preterm newborns are 1) the inherent physiologic discontinuous trace, and 2) the limited EEG reference data for the very tiny preterm infants.

The primary motivation for this work is based on the search for early recognition of impaired neuronal function as depicted by aEEG in preterm infants. This is aimed to recognize primary causes or the moment of EBA dysfunction during neonatal intensive care, and eventually to prevent or reduce permanent cerebral impairment.

Both hypoxemia and hemodynamic instability are a common threat in preterm infants and are therefore relevant matter for investigation. Hypoxemia or the lack of an appropriate oxygen supply to tissue is a multifactorial entity, primary related to impaired and/or immature respiratory condition or to right to left shunting. Preterm infants are also much more likely to have hemodynamic 
instability, which could additionally affect systemic and eventually cerebral blood flow and tissue oxygenation. The later of which can permanently impair neurodevelopmental and neurologic outcome.

In the present work, the impact of hypoxemia, hypotension or both has been first studied in both the neonatal piglet and in the fetal sheep. This animal experimentation of on-line monitoring of fetal and neonatal EBA lent the basis for the further development of the aEEG called the Maastricht Cerebral Monitor (MCM). MCM was later introduced in the NICU to study the impact of acute hypoxemia in VLBWI. Parallel to the development of MCM, CFAM3 ${ }^{\text {TM }}$ (RDM Consultants Ltd, UK), a 4th generation CFM was developed. Both devices share some aEEG characteristics and are further described in sections 2.1.2.2.2. and 2.1.2.2.3..

\subsection{Aims of the study}

1.2.1. To develop a reliable and flexible computerized aEEG for further clinical and experimental research.

1.2.2. To study the impact of acute hypoxemia, hypotension or both on EBA in both prenatal and neonatal animal models.

1.2.3. To study the role of acute hypoxemia on EBA as monitored using the MCM in VLBWI.

\subsection{Summary:}

The study of on-line continuous neonatal EBA may be clinically relevant in terms of long-term neurologic development in both preterm and term newborns. However, it has been rather limited to assess outcome in asphyctic term infants.

This work describes basic scientific and practical results obtained of the study on perinatal EBA. It involves experimental and clinical research on EBA in relation with hypoxemia and hypotension in both fetal and neonatal animals, and in VLBWI. Particular attention is paid to the development of the aEEG monitor named the Maastricht Cerebral Monitor (MCM).

This work is a joint venture between the departments of neonatology, child neurology and clinical neuro-physiology of the University Hospital Maastricht. 


\section{References}

Guyer B, Freedman MA, Strobino DM, Sondik EJ. Annual summary of vital statistics: trends in the health of Americans during the 20th century. Pediatrics. 2000; 106(6): 1307-17.

Hack M, Wilson-Costello D, Friedman H, Taylor GH, Schluchter M, Fanaroff AA. Neurodevelopment and predictors of outcomes of children with birth weights of less than 1000 g: 1992-1995. Arch Pediatr Adolesc Med. 2000; 154(7): 725-31.

Maynard D, Prior PF, Scott DF. A continuous monitoring device for cerebral activity. Electroencephalogr Clin Neurophysiol. 1969; 27(7): 672-3.

Maynard DE. Removal of EKG from fetal CFM recordings. Ann Anesthesiol Fr. 1979a; 20(3): 243-6.

Maynard DE, Cohen RJ, Viniker DA. Intrapartum fetal monitoring with the cerebral function monitor. Br J Obstet Gynaecol. 1979b; 86(12): 941-7.

Schmidt B, Davis P, Moddemann D, Ohlsson A, Roberts RS, Saigal S, Solimano A, Vincer M, Wright LL. Long-term effects of indomethacin prophylaxis in extremely-low-birth-weight infants. N Engl J Med. 2001; 344(26): 1966-72.

Vohr BR, Wright LL, Dusick AM, Mele L, Verter J, Steichen JJ, Simon NP, Wilson DC, Broyles S, Bauer CR, Delaney-Black V, Yolton KA, Fleisher BE, Papile LA, Kaplan MD. Neurodevelopmental and functional outcomes of extremely low birth weight infants in the National Institute of Child Health and Human Development Neonatal Research Network, 1993-1994. Pediatrics. 2000; 105(6): 1216-26. 
CHAPTER 2.

\section{AMPLITUDE INTEGRATED EEG (aEEG): REVIEW OF THE LITERATURE}

2.1. Electroencephalography (EEG) in perinatology:

2.1.1. The origin and characteristics of electrical brain activity

2.1.1.1. Amplitude

2.1.1.2. Spectral power (frequency)

2.1.1.3. Background activity

2.1.2. EEG devices

2.1.2.1. Conventional EEG

2.1.2.2. Amplitude-integrated EEG's

2.1.2.2.1. Cerebral Function Monitor $\left(\mathrm{CFM}^{\mathrm{TM}}\right)$

2.1.2.2.2. Cerebral Analyzing Function Monitor (CFAM ${ }^{\mathrm{TM}}$ )

2.1.2.2.3. Maastricht Cerebral Monitor (MCM)

2.2. Summary 


\section{Review of the literature}

In the following paragraphs, electric brain activity (EBA) features are discussed in relation to maturational, physiological and pathological changes. Amplitudeintegrated EEG (aEEG) devices, and its applications are further described.

\subsection{Electroencephalography in perinatology}

\subsubsection{The origin and characteristics of electric brain activity}

Introduced by Hans Berger in 1929, EEG depicts the summation of synchronous postsynaptic potentials evoked by the stimulation of radially oriented pyramidal cells within cortical gyri. These cortical postsynaptic potentials are synchronized by other nuclei, such as the thalamus (D'Allest and Andre, 2002). EEG provides electrical assessment of the cerebral cortex and other deeper structures; however, it does not represent overall brain function.

EEG characteristics comprised:

\subsubsection{Amplitude}

EEG amplitude is expressed in microvolts $(\mu \mathrm{V})$ and depends on the generation of potentials and the conductivity of the structures between the brain and scalp. Mean EEG amplitude is positive correlated with gestational age.

\subsubsection{Spectral power}

EEG contains four broad spectral bands: delta [1 - $4 \mathrm{~Hz}$ ], theta [4-8 Hz], alpha [8-14 Hz] and beta [14-30 Hz]). Frequencies of less than $7.5 \mathrm{~Hz}$ are called slow waves and those over $13 \mathrm{~Hz}$ are usually called fast waves. From a developmental point of view, slow frequencies, namely delta wave activity, are the prominent components of "at rest" neonatal EEG's. This EEG pattern develops later into a faster and dominant alpha rhythm.

\subsubsection{Background activity (continuity and discontinuity)}

Synaptogenesis, myelination, and biochemical maturation of neurons and glial cells are the basis for age-dependent electrophysiological changes (Holmes, 1986). EEG has a high correlation with neuropathologic maturational characteristics (Scher et al., 1987), fetal ultrasound parameters of maturity (Scher et al., 1994) and with the date of the last maternal menstrual period (Scher et al., 1995). EEGs of normal preterm infants at term corrected for gestational age are maturational identical to the EEGs of full-term infants (Domenech et al., 1989). One of the most impressive characteristics of the very preterm EEG is its discontinuity. The duration of the interburst intervals (IBIs) decreases as gestational age (GA) increases (Nunes et al., 1997). IBIs duration should be less than 40 seconds in infants younger than 30 weeks GA and less 
than 6 seconds in duration in full-term ones (Hahn et al., 1989). Preterm infants younger than 28-30 wk GA show an EEG pattern termed "tracé discontinu". This EEG is discontinuous with IBIs of low voltage or inactivity lasting $8-20$ sec alternating with higher amplitude and varied spectral power (Mizrahi, 1986). Another EEG pattern is the "tracé alternant", which represents the discontinuous EEG pattern of nonREM sleep in which there are runs of delta activity alternating with random faster transients. The IBIs show less voltage attenuation as that seen in tracé discontinu. Tracé alternant is seen in (near-) term infants by 36-38 wk GA (Mizrahi, 1986). By term GA, the EEG becomes gradually continuous and is almost totally synchronous at 41-44 wk conceptional age (Mizrahi, 1986). Synchrony defines the simultaneous EEG activity in analogous regions of each cerebral hemisphere. Interhemispheric synchrony increases with GA (Nunes, 1997).

\subsubsection{EEG devices}

\subsubsection{Conventional neonatal EEG (EEG)}

EEG is a multi-channel recording of EBA on paper or digitally. Within clinical practice EEG recording is usually short lasting 30 to 60 minutes. EEG is not immediately available in the NICU and has to be specially requested if clinically indicated. Furthermore, it is attended full time by an experienced EEG technician and is reviewed using unaided visual inspection by an expert neurophysiologist. Because of its multi-channel and unprocessed character, conventional EEG is the most important non-invasive tool for studying real time brain activity and the golden standard in the NICU population to date.

\subsubsection{Amplitude-integrated EEG (aEEG) monitors}

\subsubsection{1.}

\section{Cerebral function monitor (CFM ${ }^{\mathrm{TM}}$, Lectromed Devices Ltd., UK)}

In the late 1960's, Maynard, Prior and Scott provided the first description of CFM, a one-channel continuous aEEG, as a method of continuous monitoring of EBA in three resuscitated adults (Maynard et al. 1969a). This device puts on paper a processed single-channel EEG recording using a biparietal derivation covering the triple cerebral arterial boundary zone. This proved to be the least contaminated by artifacts and allows routine care. The EEG signal is amplified, filtered and time compressed. Amplitude is plotted in a semilogarithmic microV scale, which allows the overall trend of EBA to be followed and for very low voltage EEG amplitude to be identified. The plotted CFM signal appears as a band, representing the range of voltages of the EBA. The maximal and minimal amplitude excursions, and the amplitude bandwidth (difference between maximal and minimal EEG amplitude in a time window) in $\mu \mathrm{V}$ are also registered. The recording paper speed is 6,30 or $60 \mathrm{~cm} / \mathrm{h}$. The electrical activity registered with CFM has been shown to be consistent, reliable and 
quite comparable to EEG, and has been further developed in perinatology. Research on this field has mainly been concentrated on the study of full-term hypoxic-ischemic encephalopathy (HIE).

aEEG compared to multi-channel EEG:

Bjerre et al., comparing aEEG and EEG in peripartal asphyctic term $(n=13)$ and preterm infants $(n=13)$, found that aEEG recording matches with that of multi-channel EEG (Bjerre et al., 1983). Similar findings were reported by several authors (Hellström-Westas, 1992a; Greisen, 1994; al Naqeeb et al., 1999; Klebermass et al., 2001). Toet et al. successfully addressed this controversial issue in (near) term neonates with HIE and/or suspected seizures $(n=36)$. This prospective study showed a close interobserver agreement for aEEG and EEG, as well as a good correlation between the two techniques on both background and ictal activity (Toet et al., 2002).

Practical advantages of aEEG compared to multi-channel EEG:

Two important advantages of aEEG in contrast with conventional EEG when applied in the NICU are:

1. Compact aEEG traces are relative simple to interpret, allowing for evaluation of EEG changes by caregivers with no previous EEG experience and allowing faster review of long periods of recording.

2. Unrestricted access of aEEG recording makes it immediately available at the cotside.

Limitations of aEEG:

Inherent to the compact format of aEEG, however, is the loss of precision and fine diagnosis as attained by conventional EEG. For instance, localized ictal activity restricted to the occipital area, localized delta activity, etc., which required multiple channels, are not defined by aEEG (Hellstrom-Westas, 2002; Toet et al., 2002). Nor are short seizures of less than $30 \mathrm{sec}$ detected using aEEG (Hellström-Westas, 1992a; Murdoch -Eaton et al., 1994; Toet et al., 2002). 


\section{aEEG patterns:}

The interpretation of aEEG is based on the recognition of patterns. The most important patterns in infants are provided here to illustrate their characteristics:

1. Continuous normal aEEG in term infant (fig. \# 1).

2. Discontinuous normal aEEG in extreme preterm infant (fig. \# 2).

3. Burst-suppression (BS) (fig. \# 3).

4. Saw-tooth (fig. \# 4): typical pattern of intermittent seizure activity. However, no saw- tooth pattern but high voltage background activity is depicted when seizure activity is continuous. If this is the case, simultaneous conventional EEG is mandatory for diagnosis (Hellstrom-Westas L, 2002).

5. Sleep-wake states: Coherent sleep-wake state patterns using aEEG have been reported in stable premature infants older than 32-34 wk GA (Viniker et al., 1984; Hellstrom- Westas et al., 2001; Westrup B et al., 2002).

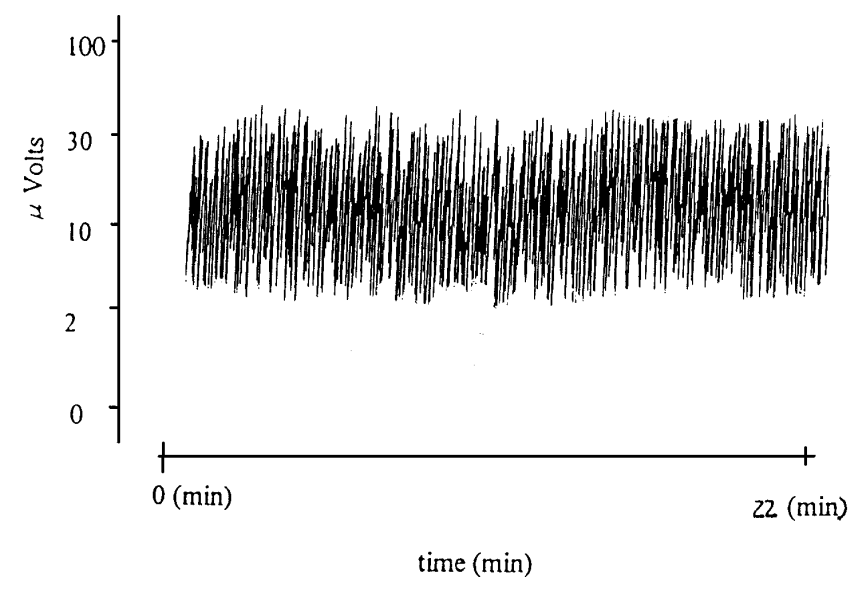

Fig.1 Continuous normal voltage aEEG measured measured by MCM in a full-term infant.

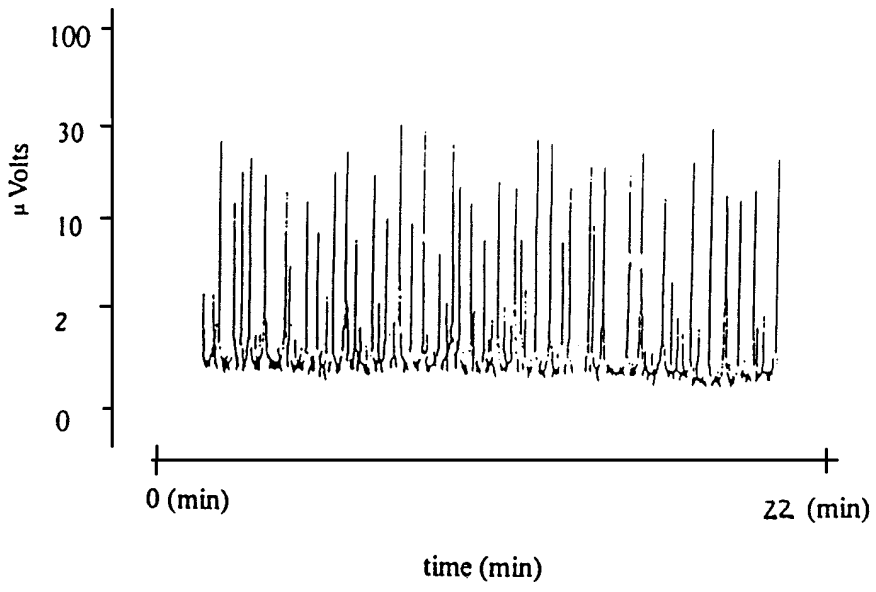

Fig. 2 Normal discontinuous aEEG by MCM in a 25 weeks GA infant. 


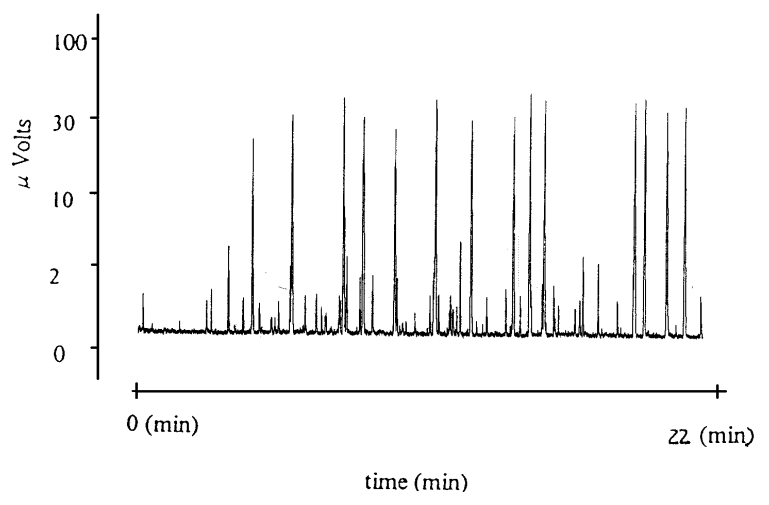

Fig. 3 Burst-suppression (BS) pattern recorded by MCM in term HIE.

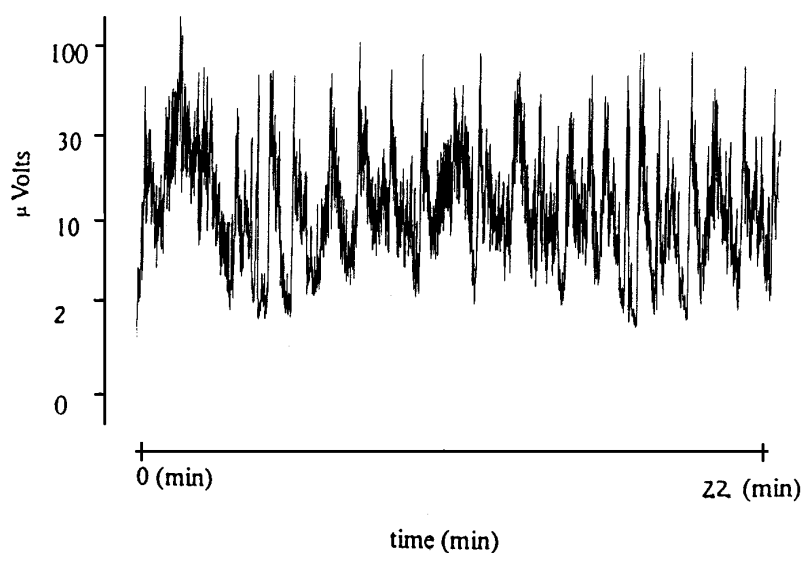

Fig.4 Saw-tooth pattern of intermittent seizure activity recorded by MCM in term HIE.

$\underline{\text { Reported neonatal and perinatal clinical situations studied by } \mathrm{aEEG}}$

The first application of aEEG in perinatology was also reported by Maynard et al., in 15 term fetuses during labor for periods of up to $6.5 \mathrm{~h}$. The authors studied the influence of maternal medication and fetal distress on causing a depression on the fetal EBA (Maynard et al., 1979a and 1979b). The work of Bjerre et al., presented the first characterizations of aEEG patterns in preterm and term infants ( 26 to 42 wk $G A, n=28$ ), however this study focused on the impact of HIE on EBA (data are discussed below) (Bjerre et al., 1983). Since then, many other authors have discussed and reported the influence of gestational age on EBA as depicted by aEEG. Viniker et al., studying a population aged 29.5 to 43 wk GA $(n=107)$, introduced a method of quantification of aEEG records (Viniker et al., 1984).

The same year, other authors studied 49 normal preterm and full-term infants 
to establish normal parameters of aEEG amplitude at different sleep-wake states between 28 to 40 wk GA (Verma et al., 1984). More recently, Thornberg and Thiringer, have further establish normal aEEG patterns at different GA (30 to 37 wk $G A, n=19)$. They showed that a gradual increase in the minimal aEEG amplitude takes place with increasing GA, resulting in a narrowing of aEEG bandwidth (Thornberg \& Thiringer, 1990). Finally, Hellstrom-Westas et al. underlined the concept that an increase in continuous aEEG background activity is positively correlated with GA in preterm infants with GA ranging from 23 to 31 wk ( $n=31)$ (Hellstrom-Westas L, 1990).

\section{$\underline{\text { Hypoxic-ischemic encephalopathy }}$}

Bjerre et al. (1983) published the first description of aEEG recording including both neonates and the impact of perinatal asphyxia in preterm and term infants aged 26 to 42 wk GA ( $n=28)$. Similar aims were reported by Archbald et al. (1984) in a cohort of 31 infants aged 23 wk to term GA, and by Klebermass et al. (2001). The resulting conclusion from these studies included the finding that significant changes in EBA, such as seizure activity, depressed and/ or silent background activity, characterized severe HIE.

As reported by Thornberg and Ekstrom-Jodal in 1994, a strong association has been observed between early aEEG patterns and long-term neurological prognosis in (near) full-term infants. Regardless of the study, persistent discontinuous electrical activity has the strongest relationship with a poor longterm outcome up to 6 years of age (Thornberg and Ekstrom-Jodal, 1994; Eken et al., 1995; Hellstrom-Westas et al., 1995; Thornberg et al., 1995; al Naqeeb et al., 1999). In addition, severe EBA dysfunction depicted as soon as 3 hours of birth predicts poor neurological outcome at 1 to 6 years of age $(n=68)$ with a positive predictive value of 0.78 and a negative predictive value of 0.84 (Toet et al., 1999). Therefore, aEEG is mostly used as a tool to depict neuronal dysfunction in asphyctic term infants.

Thornberg et al. considered in their work the analysis of neuron specific enolase (NSE) in serum and CSF and its correlation with early aEEG patterns and outcome in post-asphyctic term infants $(n=22)$. They demonstrated that high CSF-NSE levels and aEEG patterns of BS and/or seizures are associated with a poor outcome at the age of 18 months (Thornberg et al., 1995).

Recent contributions to the study of EBA in term HIE using aEEG have focused on the application of neuroprotective interventions. Allopurinol, a xanthine-oxydase inhibitor given at $40 \mathrm{mg} / \mathrm{kg}$ in two dosis within $4 \mathrm{~h}$ of birth, derived EBA and cerebral blood volume stability between 4 and $8 \mathrm{~h}$ postnatally in (near) term infants with severe birth asphyxia $(n=11)$ when compared to controls (van Bel et al., 1998). In addition, trials on post-asphyctic hypothermia used aEEG patterns to recognize eligible infants. The authors identified (near) term infants suffering severe HIE by aEEG and included them for therapeutic brain or systemic cooling (Azzopardi et al., 2000 [n=16]; Thoresen \& Whitelaw, 2000 [n=8]; Gunn et al., 2002 [n=235]). 


\section{Prognosis based on aEEG in non-HIE pathologies}

Data by Hellstrom-Westas et al. published in 1990 and 1998 provides an introductory look at the prognostic value of aEEG in non-HIE patients. They showed that serial aEEG within the first week of life in preterms infants with periventricular - intraventricular hemorrhage (PIVH) aged 23 to 32 wk GA (both studies, $n=58$ ) differentiated neurological outcome at the age of 14 months to 11 years.

Determinants of poor neurological outcome amongst survivors were seizures and suppressed aEEG background and severe PIVH as detected using head ultrasound.

\section{Seizures}

Diagnosis of clinical and silent seizures:

Preterm infants are prone to seizures as opposed to full term infants. In this context, immaturity of excitatory-inhibitory neuroransmission can increase seizure initiation and seizure maintenance (D'Allest and Andre, 2002). Furthermore, in contrast to older infants, newborn infants show electro-clinical dissociation or silent seizure activity. If this is the case, there is no clinical correlation with the electric seizure activity. Electro-clinical dissociation occurs in 19 to $79 \%$ of infants suffering from neonatal seizures (Clancy et al., 1988; Weiner et al., 1991). Therefore, continuous EEG monitoring may assess the real seizure frequency during the neonatal period (Clancy et al., 1988). This is of particular clinical importance because: 1) Visual inspection of non-paralyzed infants underrates seizure frequency (Hellstrom-Westas et al., 1985), and because 2) Antiepileptic drugs (AED) eliminate clinical but not necessarily electrical seizures (Hellstrom-Westas et al., 1988; Hakeem and Wallace, 1990). In addition, Hellstrom-Westas showed that seizure activity is extremely frequent $(75 \%)$ in infants suffering PIVH and that silent seizures were more frequent (87\%) than those clinically observed (Hellstrom-Westas L, 1990).

Moreover, few studies systematically describe monitoring the effect of lidocaine and other anticonvulsants on EBA using aEEG (Hellstrom-Westas et al., 1988; Hellstrom-Westas et al., 1992b).

\section{aEEG changes during surfactant administration}

Hellstrom-Westas et al. (1989) presented the first case-report linking the effect of surfactant administration with depressed EEG in one infant aged 28 wk of gestation. Later, other authors have tried to clarify this phenomenon:

A significant fall in mean arterial pressure (MAP) with an unchanged cerebral blood flow (CBF) measured by Near Infrared Spectroscopy (NIRS) or xenon clearance $\left({ }^{133}\right.$ Xe clearance) was observed in preterm infants $(n=34)$ after a single dose of $200 \mathrm{mg} / \mathrm{kg}$ porcine surfactant. EBA was depressed for 10 to 20 minutes after surfactant administration despite an intact CBF (Skov et al., 1992).

Hellstrom-Westas et al. reported similar findings in preterm infants aged 25 to 
33 wk GA $(n=23)$. This study showed a transient and significant fall in MAP of $9.3 \mathrm{~mm} \mathrm{Hg}$ after the instillation of porcine surfactant at $2.5 \mathrm{ml} / \mathrm{kg}(200 \mathrm{mg} / \mathrm{kg})$ in one or two aliquots after deconnecting the ventilator. This effect lasted for less than 10 minutes and occurred simultaneously with a depressed background EEG activity. The aEEG pattern, primarily discontinuous at these GA's, depicted less burst rate. The authors hypothesized that the EBA changes were related to hemodynamic instability added to the effect of oxygen free radicals or other not specified vasoactive substances (Hellstrom-Westas et al., 1992c).

Bell et al., however, found no correlation between a fall in MAP $(3.2 \mathrm{mmHg}$ or $10 \%$ ), plasma hypoxanthine levels and EEG depression after porcine surfactant therapy. As demonstrated by the same authors as above, CBF as measured by ${ }^{133}$ Xe clearance remained unchanged (Skov et al., 1992; Bell et al., 1994).

The issue further explored by Lundstrom \& Greisen was whether gradual hemodynamic changes resulted in less important EBA suppression. Twenty preterm infants with GA below 34 wk received two or six aliquots of porcine surfactant $(200 \mathrm{mg} / \mathrm{kg})$. Reoxygenation was similar in the two groups however the fall in MAP was less abrupt in those infants receiving six aliquots. The aEEG depression in terms of less burst rate activity lasting 15 to 20 minutes was however similar in both groups. The authors concluded that cerebral ischemia is not the primary cause of EBA changes and suggested that this might be due to systemic and cerebral vasodilation (Lundstrom \& Greisen, 1996).

Taken together, the finding that surfactant administration is able to depress EBA calls the cerebral ischemia hypothesis into question. The specific mechanisms remain unknown and deserve further investigation.

\section{Other pathologies studied by aEEG}

Periventricular-intraventricular hemorrhage (PIVH):

Greisen (1987) and Klebermass (2001) analyzed the EEG background activity of preterm infants suffering from PIVH. The authors observed depressed EBA and seizure activity. Generally speaking, the EBA background was depressed in infants which showed brain parenchymal pathology. Abnormalities in neonatal EEG are in general not specific to etiological diagnosis (Holmes and Lombroso, 1993).

\section{Systemic hypotension:}

Greisen et al. studied 24 ventilated preterm infants younger than 32 wk GA with hypotension using aEEG and internal carotid blood flow velocity. After volume expansion and subsequently normalization of the blood pressure, they still found a reversible EEG depression (less burst rate) (Greisen et al., 1988). One year later, the same authors studied the relationship between EBA and CBF in twenty infants aged 27 to 32 wk GA by combining aEEG and the xenon clearance technique. They showed that EBA was detectable with CBF as low as $5 \mathrm{ml} / 100 \mathrm{~g} / \mathrm{min}$. They further suggested that CBF of less than $8 \mathrm{ml} / 100 \mathrm{~g} / \mathrm{min}$ probably indicated impaired brain perfusion (Greisen et al., 1989).

Hellstrom-Westas et al., 1989 deals with the impact of acute hemodynamic instability as caused by tension pneumothorax in one ventilated term infant. 
EBA was extremely depressed during the air leak and normalized after pleural drainage.

Hypoglycemia:

Westas (1989) discussed the implications of hypoglycemia upon EBA. One septic term patient is described. The authors found reversible EEG seizure and depressed activity during hypoglycemia. In addition, other authors used aEEG to ascertain the influence of mild asymptomatic hypoglycemia on EBA in term infants born to diabetic mothers $(n=12)$. EBA was not affected (Stenninger et al., 2001).

\section{Sedation:}

In general, Bell et al. studied the effect of sedation on EBA $(n=77)$, and showed that a continuous drip of phenobarbital and morphine prolonged IBIs. A single dose of diazepam used as premedication for endotracheal intubation increased EEG depression. More specifically, the authors argued for the cautious interpretation of aEEG recording in sedated patients (Bell et al, 1993).

\section{Exchange transfusion:}

Thirteen (near) term infants $(36.3 \pm 1.9$ wk GA) were subjected to doublevolume exchange transfusion because of hyperbilirubinemia as caused by hemolysis, while measuring MAP and aEEG. The authors showed that EEG amplitude decreased by less than $6 \%$ as compared to baseline EEG during a 20-ml blood withdrawal. They concluded however, that the EEG changes were probably not clinically relevant (Benders et al., 2000).

\section{Neonatal animal experimentation}

Near term and newborn lambs:

Shadid et al. studied the effect of allopurinol, indomethacine, deferoxamine and their combination in newborn lambs aged $4-10$ days $(n=35)$ subjected to hypoxemia and systemic hypotension (FiO2 0.06 to 0.08 during 30 minutes followed by exsanguination to induce a MAP of $<35 \mathrm{mmHg}$ ). The authors showed a recovery of EBA in all antioxidative therapy groups. Further, there was no additional effect when the drugs were combined (Shadid et al., 1998).

No significant modification of ECBA as measured by aEEG was observed in anesthetized newborn lambs aged 4-9 days $(n=12)$ after 10 minutes of hypoxemia (FiO2 0.08), nor after exsanguination providing a MAP greater than $30 \mathrm{mmHg}$ (van de Bor et al., 1999).

Ten newborn lambs aged 8-10 days were subjected to a double volume exchange transfusion. Blood was withdrawn in aliquots of $20 \mathrm{ml}$. The authors showed a decrease in carotid flow whilst a small and not significant mean EBA decline was present (Benders et al., 1999).

Thorngren-Jerneck et al. presented the approach of combining postnatal cerebral glucose metabolism (CMRgl) as measured by PET, with the EBA recording of aEEG in asphyctic fetuses. Near term fetal lambs $(n=8)$ showed a reduced brain glucose metabolism and an abnormal EBA 4 hours after $14.5 \pm$ 
4.2 minutes of umbilical cord occlusion and cardiac arrest. MAP, blood gases and serum glucose were normal. The authors concluded that a global reduction of CMRgl can be an early indicator of HIE (Thorngren-Jerneck et al., 2001).

Piglets:

Peeters-Scholte et al. described the impact of one-hour of bilateral carotid occlusion and hypoxia as well as the effect of pharmacological post-HIE intervention using saline, allopurinol, deferoxamine or 2-iminobiotin in newborn piglets aged 1 to 3 days $(n=43)$. EBA, cerebral hemodynamics and cerebral energy status as measured by aEEG, NIRS and phosphorus Magnetic Resonance Spectroscopy, respectively were significantly less affected in the intervention groups compared to controls. aEEG was better preserved in the 2iminobiotin-treated group (Peeters-Scholte et al., 2002).

Guinea pigs:

Yuan et al. subjected spontaneously breathing guinea pigs aged between 1-4 days $(n=36)$ to a decrease in $\mathrm{FiO} 2$ of 0.09 or 0.06 for 10 minutes. According to this work, EBA depression was more consistent with $0.06 \mathrm{FiO} 2$. In addition, the authors showed that the animals became apnoeic in response to hypoxia before the complete suppression of ECBA. This suggested that the respiratory "switching-off" mechanisms could be activated before severe cortical depression (Yuan et al., 1995).

\subsubsection{2.}

\section{Cerebral function analysing monitor (CFAM ${ }^{\mathrm{TM}}$, RDM Consultants Ltd, UK)}

Based on aEEG, the development of CFAM includes improvements in both device technology and software. In addition to providing amplitude integrated EEG patterns, CFAM technology introduced EEG spectral power analysis. To the present day, 3 generations of CFAM devices have been developed and commercialized. Their specific characteristics are described below:

CFAM 1 (First generation, first report in 1979): a one-channel, chart paper recording, controlled by a microprocessor. CFAM produces paper traces of the aEEG, EEG frequency percentage, percentage time-suppression below $1 \mu \mathrm{V}$, scalp muscle activity and electrode impedance. Evoked potentials could be computed (Maynard, 1979c, Sebel et al., 1983; Maynard \& Jenkinson, 1984). EEG frequency percentage is calculated using a modified zero crossing technique.

CFAM 2 (Second generation, RDM Consultants Ltd., UK, 1989) One or two-channel CFAM, DOS based system, digital signal processor for filtering and Fast Fourier Transform algorithm (FFT), which estimates EEG background spectral content. Also depicts unprocessed EEG and includes offline computerized statistical analysis. 
CFAM 3 (Third generation, RDM Consultants Ltd., UK, 1995)

Up to four-channel CFAM, DOS based CFAM1 system. In addition, CFAM 3 also depicts unprocessed EEG and allows real-time and off-line data analysis.

\section{Differences and advantages of CFAM as compared to aEEG}

1. CFAM depicts EEG frequency percentage and evoked potentials.

2. Two or more channel EEG allows the study of EEG symmetry and synchrony.

3. Digital acquisition and storage.

4. Computerized analysis and post recording evaluation, allows change in the time base of the recording speed.

\section{Limitations of CFAM}

(See aEEG limitations in section 2.1.2.2.1.)

$\underline{\text { Reported neonatal and pediatric clinical situations studied by CFAM }}$

Since its development, CFAM studies have focussed on the adult population whilst very few studies have addressed the use of CFAM in the pediatric clinic. The main paradigm studied is that of pediatric seizures. Three authors have stressed the importance of studying electric seizures using CFAM (Tasker et al., 1989a; Tasker et al., 1989b; Crawley et al., 1996).

Murdoch Eaton et al. showed in a retrospective study that asymmetrical EEG recordings, suppression of background activity or electrical seizures were predictive of poor outcome at the age of 2 years in an heterogeneous pediatric population aged 2 weeks to 16 years (median 1.7 years, $n=108$ ) (Murdoch Eaton et al., 2001).

\subsection{Maastricht cerebral monitor (MCM)}

The effort of our group has focussed on the need for flexibility and simplicity of EEG acquisition and data interpretation within the NICU. MCM software responds to this need, as discussed in chapter 3. CFM/CFAM have provided the basis for the development of the MCM. This technique combines some of the best features of aEEG and CFAM. Detailed technical considerations are described in chapter 3 of this thesis (Reulen et al., 1999). In brief, the MCM characteristics are:

MCM uses the software Poly (Inspektor Research System ${ }^{\circledR}$, Amsterdam, NL) for digital EEG acquisition, data storage and review.

MCM depicts three-channels of EEG electrodes $\left(P_{3}-F_{z} ; P_{4}-F_{z ;} P_{3}-P_{4}\right)$ as unprocessed EEG, EEG amplitude and absolute as well as relative frequency domain. Epochs of 2 or 3.6-seconds are analysed by the computer yielding EEG amplitude (bandwidth, minimum and maximum mean), frequency percentages and absolute powers in the delta, theta, alpha and beta signal frequency range. MCM also displays the percentage time-suppression and allows real-time and 
post recording change of the time base for registration and analysis. MCM does however not include evoked potential recording.

\section{Advantages of MCM compared to CFM/CFAM:}

In addition to the reliability and practical ease of use of the CFAM software, MCM includes the following parameters that make its use more flexible:

1. Asymmetry, mean and $90 \%$ edge frequency and absolute spectral power analysis.

2. Off-line replay of the recording.

3. On- and off-line storage and editing of comments.

Reported applications and clinical situations studied by MCM:

MCM's applications are extensively discussed in chapters 4 to 8 of this thesis.

\subsection{Summary:}

1. aEEG allows long-term, flexible and reliable EBA recording in the NICU.

2. Currently, aEEG has turned into a reliable prognostic tool for use in asphyctic term infants and eventually in those who are suitable for neuroprotective therapies.

3. The study of continuous aEEG both in preterm infants and as a long-term monitoring tool is limited.

4. aEEG is complementary and inseparably bound with conventional multichannel serial EEG.

The following chapters emphasize our experience with perinatal experimental aEEG monitoring and finally report our experience in VLBWI. 


\section{References}

al Naqeeb N, Edwards AD, Cowan FM, Azzopardi D. Assessment of neonatal encephalopathy by amplitude-integrated electroencephalography. Pediatrics 1999; $103(6$ Pt 1): 1263-71.

Archbald F, Verma UL, Tejani NA, Handwerker SM. Cerebral function monitor in the neonate. II: Birth asphyxia. Dev Med Child Neurol. 1984; 26(2): 162-8.

Azzopardi D, Robertson NJ, Cowan FM, Rutherford MA, Rampling M, Edwards AD. Pilot study of treatment with whole body hypothermia for neonatal encephalopathy. Pediatrics. 2000; 106(4): 684-94.

Bell $\mathrm{AH}$, Greisen $\mathrm{G}$, Pryds $\mathrm{O}$. Comparison of the effects of phenobarbitone and morphine administration on EEG activity in preterm babies. Acta Paediatr. 1993 ; 82(1): 35-9.

Bell AH, Skov L, Lundstrom KE, Saugstad OD, Greisen G. Cerebral blood flow and plasma hypoxanthine in relation to surfactant treatment. Acta Paediatr. 1994; 83(9): 910-4.

Benders MJ, Meinesz JH, van Bel F, van de Bor M. Changes in electrocortical brain activity during exchange transfusions in newborn infants. Biol Neonate. $2000 ; 78(1): 17-21$.

Bjerre I L, Hellström-Westas I, Rosén NW, Svenningsen. Monitoring of cerebral function after severe asphyxia in infancy. Arch Dis Child 1983; 58: 997-1002.

Clancy RR, Legido A, Lewis D. Occult neonatal seizures. Epilepsia. 1988; 29(3): 256-61.

Crawley J, Smith S, Kirkham F, Muthinji P, Waruiru C, Marsh K. Seizures and status epilepticus in childhood cerebral malaria. QJM. 1996; 89(8): 591-7.

DÁllest $A M$ and Andre M. Electroencephalography. In: The newborn brain: neuroscience and clinical applications. Ed. Lagercrantz $\mathrm{H}$ et al. Cambridge University Press 2002, Cambridge - UK

Domenech E, Castro R, Casabona C, Mendez A, Ormazabal C, Rodriguez Luis JC. Electroencephalographic maturation in preterm newborn infants. An Esp Pediatr. 1989; 31(3): 221-8.

Eken P, Toet MC, Groenendaal F, de Vries LS. Predictive value of early neuroimaging, pulsed Doppler and neurophysiology in full term infants with hypoxic-ischaemic encephalopathy. Arch Dis Child 1995;73: F75-F80. 
Gavilanes AWD, J.Vles, R.Van Sprundell, K.Von Siebenthal, F.Nieman, J.Reulen, CE.Blanco Electrocortical brain activity, cerebral haemodynamics and oxygenation during progressive hypotension. Clinical Neurophysiology $2001 ; 112(1): 52-59$.

Gotman J, Flanagan D, Rosenblatt B, Bye A, Mizrahi EM. Evaluation of an automatic seizure detection method for the newborn EEG.

Electroencephalogr Clin Neurophysiol. 1997; 103(3): 363-9.

Greisen G, Hellstrom-Westas L, Lou H, Rosen I, Svenningsen NW. EEG depression and germinal layer haemorrhage in the newborn. Acta Paediatr Scand. 1987; 76(3): 519-25

Greisen G, Pryds O, Rosen I, Lou H. Poor reversibility of EEG abnormality in hypotensive, preterm neonates. Acta Paediatr Scand. 1988; 77(6): 785-90.

Greisen G, Pryds O. Low CBF, discontinuous EEG activity, and periventricular brain injury in ill, preterm neonates. Brain Dev. 1989; 11(3): 64-8.

Greisen G. Tape-recorded EEG and the cerebral function monitor: amplitudeintegrated, time-compressed EEG. J.Perinat.Med.1994; 22:541-546.

Gunn A, Heymann M, Gluckman P, Wyatt J. Safety of brain cooling with mild systemic hypothermia for the treatment of perinatal hypoxic-ishemic encephalopathy: The Cool Cap Trial. Abstract Proceedings Hot Topic in Neonatology. Washington DC 2002.

Hahn JS, Monyer $\mathrm{H}$, Tharp BR. Interburst interval measurements in the EEGs of premature infants with normal neurological outcome. Electroencephalogr Clin Neurophysiol. 1989; 73(5): 410-8.

Hakeem VF, Wallace SJ. EEG monitoring of therapy for neonatal seizures. Dev Med Child Neurol. 1990; 32(10): 858-64.

Hellstrom-Westas L, Rosen I, Swenningsen NW. Silent seizures in sick infants in early life. Diagnosis by continuous cerebral function monitoring. Acta Paediatr Scand. 1985; 74(5): 741-8.

Hellstrom-Westas L, Westgren U, Rosen I, Svenningsen NW. Lidocaine for treatment of severe seizures in newborn infants. I. Clinical effects and cerebral electrical activity monitoring. Acta Paediatr Scand. 1988; 77(1): 79-84. Hellstrom-Westas L, Rosen I, Svenningsen NW. Cerebral complications detected by EEG-monitoring during neonatal intensive care. Acta Paediatr Scand Suppl. 1989; 360:83-6.

Hellstrom-Westas L, Rosen I, Svenningsen NW. Cerebral function monitoring during the first week of life in extremely small low birthweight (ESLBW) infants. Neuropediatrics. 1991; 22(1): 27-32. 
Hellström-Westas L. Comparison between tape-recorded and amplitude-integrated EEG monitoring in sick newborn infants. Acta Paediatr.Scand.1992a; 81:812-819.

Hellstrom-Westas L, Svenningsen NW, Westgren U, Rosen I, Lagerstrom PO. Lidocaine for treatment of severe seizures in newborn infants. II. Blood concentrations of lidocaine and metabolites during intravenous infusion. Acta Paediatr. 1992b; 81(1): 35-9.

Hellstrom-Westas L, Bell AH, Skov L, Greisen G, Svenningsen NW. Cerebroelectrical depression following surfactant treatment in preterm neonates. Pediatrics. 1992c; 89(4 Pt 1): 643-7.

Hellstrom-Westas L, Rosen I, Svenningsen NW. Predictive value of early continuous amplitude integrated EEG recordings on outcome after severe birth asphyxia in full term infants. Arch Dis Child Fetal Neonatal Ed. 1995 Jan; 72(1): F34-8.

Hellstrom-Westas L, Klette H, Thorngren-Jerneck, Rosen I, Svenningsen NW. Pronostication of neurological outcome during the first days of life in preterm infants with large IVH's. Pediatr Res. 1998; 43,319A.

Hellstrom-Westas L, Inghammar M, Isaksson K, Rosen I, Stjernqvist K. Short-term effects of incubator covers on quiet sleep in stable premature infants. Acta Paediatr. 2001; 90(9): 1004-8.

Hellstrom-Westas $L$. Cerebral function monitoring. In: The newborn brain: neuroscience and clinical applications. Ed. Lagercrantz $\mathrm{H}$ et al. Cambridge University Press 2002, Cambridge - UK

Holmes GL. Morphological and physiological maturation of the brain in the neonate and young child. J Clin Neurophysiol. 1986; 3(3): 209-38.

Holmes GL, Lombroso CT. Prognostic value of background patterns in the neonatal EEG. J Clin Neurophysiol. 1993; 10(3): 323-52.

Klebermass K, Kuhle S, Kohlhauser-Vollmuth C, Pollak A, Weninger M. Evaluation of the Cerebral Function Monitor as a tool for neurophysiological surveillance in neonatal intensive care patients. Childs Nerv Syst. 2001; 17(9): 544-50.

Lundstrom KE, Greisen G. Changes in EEG, systemic circulation and blood gas parameters following two or six aliquots of porcine surfactant. Acta Paediatr. 1996; 85(6): 708-12.

Maynard D, Prior PF, Scott DF. A continuous monitoring device for cerebral activity. Electroencephalogr Clin Neurophysiol. 1969a; 27(7): 672-3. 
Maynard D, Prior PF, Scott DF. Device for continuous monitoring of cerebral activity in resuscitated patients. Br Med J. 1969b 29; 4(682): 545-6.

Maynard DE. Removal of EKG from fetal CFM recordings. Ann Anesthesiol Fr. 1979a; 20(3): 243-6.

Maynard DE, Cohen RJ, Viniker DA. Intrapartum fetal monitoring with the cerebral function monitor. Br J Obstet Gynaecol. 1979b; 86(12): 941-7.

Maynard DE. Development of the CFM: the Cerebral Function Analysing Monitor (CFAM). Ann Anesthesiol Fr 1979c; 20(3): 253-5

Maynard DE, Jenkinson JL. The cerebral function analysing monitor. Initial clinical experience, application and further development. Anaesthesia. 1984; 39(7): 678-90.

Mizrahi EM. Neonatal electroencephalography: Clinical features of the newborn, techniques of recording, and characteristics of the normal EEG. Am J EEG Technolol. 1986; 26: 81-103.

Murdoch Eaton D, Toet M, Livingston J, Smith I, Levene M. Evaluation of the Cerebro Trac 2500 for monitoring of cerebral function in the neonatal intensive care. Neuropediatrics. 1994 Jun; 25(3): 122-8.

Murdoch-Eaton D, Darowski M, Livingston J. Cerebral function monitoring in paediatric intensive care: useful features for predicting outcome. Dev Med Child Neurol. 2001; 43(2): 91-6.

Nunes ML, Da Costa JC, Moura-Ribeiro MV. Polysomnographic quantification of bioelectrical maturation in preterm and fullterm newborns at matched conceptional ages. Electroencephalogr Clin Neurophysiol. 1997; 102(3): 18691.

Peeters-Scholte C, Van Den Tweel E, loroi T, Post I, Braun K, Veldhuis W, Nicolay K, Groenendaal F, Van Bel F. Pharmacological interventions in the newborn piglet in the first $24 \mathrm{~h}$ after hypoxia-ischemiaA hemodynamic and electrophysiological perspective. Exp Brain Res. 2002; 147(2): 200-8.

Rennie JM. Neonatal seizures. Eur J Pediatr. 1997; 156(2): 83-7.

Reulen JPH, Gavilanes AWD, van Mierlo D, Blanco CE, Spaans F, Vles JSH. Computerised cerebral function analysing monitor for the neonatal intensive care unit. Journal of Medical Engineering and Technology 1999 23:1; 29-37

Sarnat HB, Sarnat MS. Neonatal encephalopathy following fetal distress. A clinical and electroencephalographic study. Arch Neurol. 1976; 33(10): 696705. 
Scher MS, Barmada MA. Estimation of gestational age by electrographic, clinical, and anatomic criteria. Pediatr Neurol. 1987; 3(5): 256-62.

Scher MS, Martin JG, Steppe DA, Banks DL. Comparative estimates of neonatal gestational maturity by electrographic and fetal ultrasonographic criteria. Pediatr Neurol. 1994; 11(3): 214-8.

Scher MS, Sinha S, Martin J, Steppe DA. Estimation of gestational maturity of preterm infants by five fetal sonographic measurements compared with neonatal EEG and the last menstrual period. Electroencephalogr Clin Neurophysiol. 1995; 95(6): 408-13.

Sebel PS, Maynard DE, Major E, Frank M. The cerebral function analysing monitor (CFAM). A new microprocessor-based device for the on-line analysis of the EEG and evoked potentials. Br J Anaesth. 1983; 55(12):1265-70.

Skov L, Bell A, Greisen G. Surfactant administration and the cerebral circulation. Biol Neonate. 1992; 61 Suppl 1: 31-6.

Stenninger E, Eriksson E, Stigfur A, Schollin J, Aman J. Monitoring of early postnatal glucose homeostasis and cerebral function in newborn infants of diabetic mothers. A pilot study. Early Hum Dev. 2001; 62(1): 23-32.

Thornberg E, Thiringer K. Normal pattern of the cerebral function monitor trace in term and preterm neonates. Acta Paediatr Scand. 1990; 79(1): 20-5.

Thornberg E, Ekstrom-Jodal B. Cerebral function monitoring: a method of predicting outcome in term neonates after severe perinatal asphyxia. Acta Paediatr. 1994; 83(6): 596-601.

Thornberg E, Thiringer K, Hagberg H, Kjellmer I. Neuron specific enolase in asphyxiated newborns: association with encephalopathy and cerebral function monitor trace. Arch Dis Child Fetal Neonatal Ed. 1995; 72(1): F39-42.

Toet MC, Hellstrom-Westas L, Groenendaal F, Eken P, de Vries LS. Amplitude integrated EEG 3 and 6 hours after birth in full term neonates with hypoxicischaemic encephalopathy. Arch Dis Child Fetal Neonatal Ed. 1999; 81(1): F1923.

Toet MC, van der Meij W, de Vries LS, Uiterwaal CS, van Huffelen KC. Comparison between simultaneously recorded amplitude integrated electroencephalogram (cerebral function monitor) and standard electroencephalogram in neonates. Pediatrics. 2002; 109(5):772-9.

Turnbull JP, Loparo KA, Johnson MW, Scher MS. Automated detection of trace alternant during sleep in healthy full-term neonates using discrete wavelet transform. Clin Neurophysiol. 2001; 112(10): 1893-900. 
Van Bel F, Shadid M, Moison RM, Dorrepaal CA, Fontijn J, Monteiro L, Van De Bor M, Berger HM. Effect of allopurinol on postasphyxial free radical formation, cerebral hemodynamics, and electrical brain activity. Pediatrics. 1998; 101(2):185-93.

Van de Bor M, Meinesz J, Benders MJ, Steendijk P, Lopes Cardozo RH, van Bel F. Electrocortical brain activity during hypoxia and hypotension in anesthetized newborn lambs. Early Hum Dev. 1999; 55(3): 237-45.

Verma UL, Archbald F, Tejani NA, Handwerker SM. Cerebral function monitor in the neonate. I: Normal patterns. Dev Med Child Neurol. 1984; 26(2): 154-61.

Viniker DA, Maynard DE, Scott DF. Cerebral function monitor studies in neonates. Clin Electroencephalogr. 1984; 15(4): 185-92.

Weiner SP, Painter MJ, Geva D, Guthrie RD, Scher MS. Neonatal seizures: electroclinical dissociation. Pediatr Neurol. 1991; 7(5):363-8.

Westrup B, Hellström-Westas L, Stjernqvist K, Lagercrantz H. No indications of increased quiet sleep in infants receiving care based on the Newborn Individualized Developmental Care and Assessment Program (NIDCAP). Acta Paediatrica 2002; 91 (3): 318-322. 
CHAPTER 3.

THE MAASTRICHT CEREBRAL MONITOR (MCM) FOR THE NEONATAL INTENSIVE CARE UNIT

J.P.H. Reulen, A.W.D. Gavilanes, D. van Mierlo, C. Blanco, F. Spaans and J.S.H. Vles

Journal of Medical Engineering and Technology 1999; 23:1; 29-37 


\section{Summary}

Although long-term monitoring of cerebral activity can be important in the neonatal intensive care, the complexity of multi-channel EEG makes it less suitable for this purpose. In the past, a cerebral function monitor (CFM) was developed (Maynard [10]; Prior and Maynard [15]) that analyses EEG. The output parameter of the CFM is a semi-logarithmic amplitude distribution resulting from the amplification, bandpass filtering, compression, rectification and smoothing of the single-channel EEG. Drawbacks of the CFM include its inflexibility and limited single-channel processing capacity and its lack of functionality for data storage, review or re-analysis. Modern computers are powerful enough that a system can be built which does not have these drawbacks. We have developed such a system: the Maastricht Cerebral Monitor (MCM). The MCM is a flexible system that not only overcomes the CFM drawbacks but also provides advanced signal analysis. It was developed with a software system (Poly) for acquisition, high quality real-time display, on-line analysis and storage of physiological signals. The MCM processes three EEG signals in the amplitude and frequency domains. Other parameters provided by the MCM are asymmetry of absolute frequency powers, \%suppression, mean and $90 \%$ spectral edge frequency. Electrode impedance is recorded as a measure of the quality of the recording.

\section{Key words}

EEG, cerebral monitor, CFM, CFAM, MCM, Poly, neonatal, intensive care

\section{Introduction}

The monitoring of cerebral electrical activity by EEG is especially important in the neonatal intensive care unit (NICU) (Holmes [9]; Altafullah [1]; Eken [3]; Hellström-Westas [8]). The majority of the newborns treated in the NICU can be regarded as 'cerebral-risk' patients.

Multi-channel EEG recording poses numerous problems for monitoring in the NICU. First of all, it requires considerable technical skill, time and expertise to interpret the on-line tracings. Secondly, the bulky equipment may impede access to the infant, and thirdly, it is difficult to apply a large number of electrodes to the relatively small area of scalp available on an infant. To increase the benefits of continuous EEG-monitoring, a system is required that is automated and gives a rapid on-line analysis and indication of cerebral function. Several devices are available that meet these demands by using data reduction techniques (Procaccio [16]; Toner [17]; Thomsen and Prior [19]). All make it obligatory to sacrifice much of the information contained in the regular EEG in order to make continuous monitoring possible.

In the past a single-channel cerebral function monitor (CFM) was developed (Maynard [10]; Prior and Maynard [15]) that output to a low-speed paper recorder a semi-logarithmic amplitude distribution resulting from the amplification, bandpass filtering, compression, rectification and smoothing of 
the original single-channel EEG. This monitor gives only a gross indication of overall cerebral activity, but it has the advantage of providing continuous cerebral surveillance with minimal interference (Thornberg and Ekström-Jodal [17]). The analysis provided by the CFM is predominantly in the amplitude domain. However, the EEG of the early neonate is characterised by transient bursts of activity of mixed frequency superimposed on a flat background (Hellström-Westas [6]). With increasing gestational age, the iso-electric intervals between bursts of activity become shorter and the voltage differences between bursts and attenuated activity during sleep become smaller (Thornberg and Thiringer [18]). These facts indicate that frequency analysis is invaluable in the interpretation of the maturing EEG, something that the CFM can't provide. Other major drawbacks of the CFM are its inflexibility, its limited one-channel processing capacity and its lack of functions supporting data storage, review and re-analysis. Nowadays a modern system for cerebral monitoring can overcome these limitations. This paper describes the main properties of one such newly designed computerised NICU cerebral function monitor: the Maastricht Cerebral Monitor (MCM).

\section{Description of the original CFM}

The original cerebral function monitor, the CFM, has been available now for over 20 years and was originally constructed for use in adult patients. The CFM provides a filtered, integrated and compressed EEG signal as a single trace from a pair of parietal electrodes $\left(\mathrm{P}_{3}\right.$ and $\left.\mathrm{P}_{4}\right)$ (Maynard [10]). These parietal electrodes were selected for several reasons: parietal EEG amplitude is relatively large both during wake and sleep, and while this region is sensitive to minor muscle potential and/or eye-movement disturbances, placement in this region does not interfere with nursing procedures, it doesn't lead to pressure sores, and finally, it covers the area of perfusion by those arteries most vulnerable to ischaemic brain damage. The output parameter is recorded with a time compression factor of about 500 . The electrode impedance is continuously measured and written out in order to provide a measure of the technical quality of the recording. The filtered EEG signal is rectified, smoothed and amplitudeintegrated before it is registered on heat-sensitive paper (slow speeds of 6 or $30 \mathrm{~cm}$ /hour) with a semi-logarithmic amplitude scale running from 0 to $100 \mu \mathrm{V}$. This scale makes the CFM more sensitive to activity in the low voltage range without the need for gain adjustment. The CFM makes long-term monitoring possible for hours or even days. This is achieved by first filtering the original EEG signal with an inverse $U$-shaped bandpass filter which has a maximum at $10 \mathrm{~Hz}$ and declines sharply below $2 \mathrm{~Hz}$ and above $16 \mathrm{~Hz}$, and then rectifying and smoothing the instantaneous integrated amplitude with a smoothing time constant of $0.3 \mathrm{~s}$. The signal processing performed in the CFM is summarised in the block diagram of Fig.1.

Comparison of the CFM output with the traditional EEG has shown that CFM can signal significant alterations in the functional state of the brain (Prior \& Maynard [15]; Hellström-Westas [7]; Greisen [5]). A major advantage of the CFM is that the direct availability of the record makes prompt intervention 
possible in occurrences of seizure activity or other disturbances of brain function. The interpretation is based mainly on pattern recognition and is relatively easy to carry out once the limitations of the instrument are understood (Viniker [21]). The trace appears as a band that can be characterised by width and mean value. For example, in the case of "burst-suppression" activity, i.e., intermittent bursts of activity separated by periods of electrical silence, the output is a low-level band with a comb-like appearance (see Fig.2). The lower edge of the band corresponds to the lowest amplitude of the EEG signal and the width of the band indicates the variations in the minimum and maximum voltages of the cerebral electrical activity.

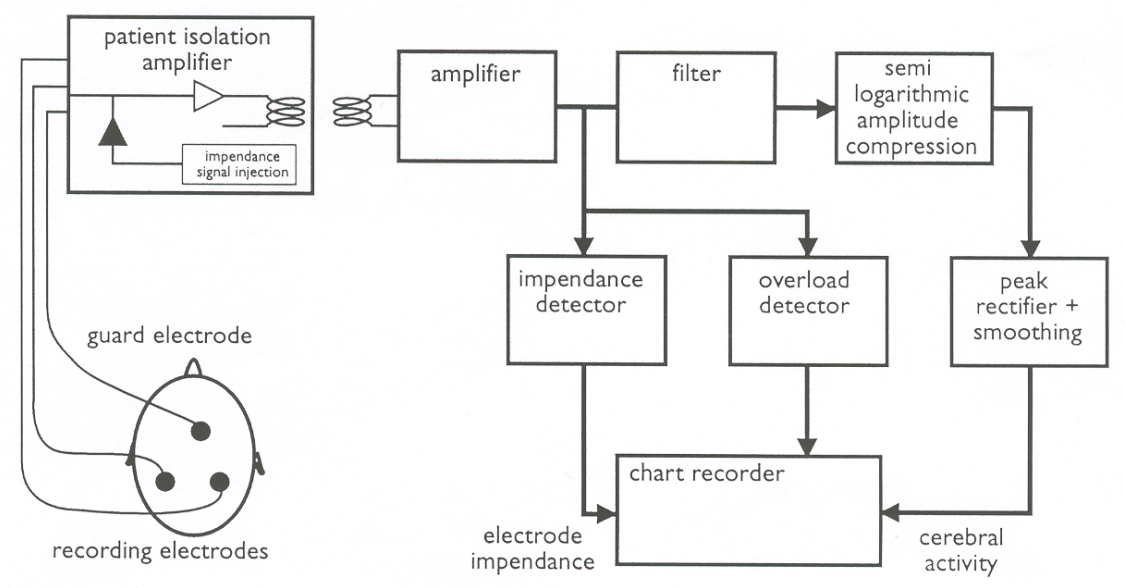

Fig. 1. Block diagram of single-channel EEG signal processing in the original CFM (from Prior and Maynard, 1986).

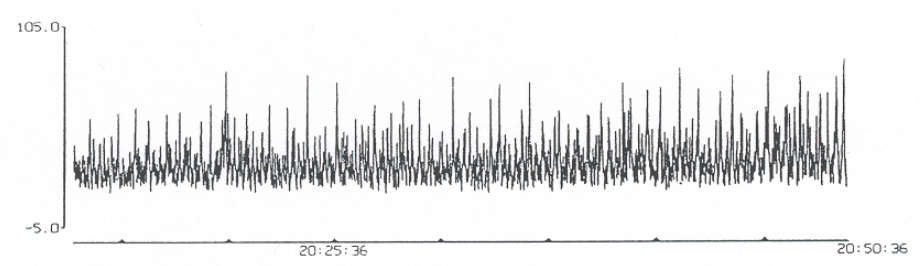

Fig. 2. Example of a CFM signal as a function of time (amplitude in $\mu \mathrm{V}$ ). 


\subsection{Major drawbacks of the original CFM}

The major drawbacks of the original CFM can be summed up as follows: - montage: the $\mathrm{P}_{3}-\mathrm{P}_{4}$ recording reflects instantaneous differences in electric potentials between the two parietal regions. Hence, in situations with interhemispheric asymmetry, the one-channel CFM could be falsely normal. Furthermore, a one-channel EEG recording as a marker of cerebral activity is rather limiting.

- no frequency analysis : the recording contains no information on frequency content.

- no re-analysis/review : there is no facility to store and to re-analyse the data, to perform statistical computations on the data or to review the data off-line in another mode of presentation.

- no signal combination: EEG parameters cannot be combined with other physiological signals such as heart rate, respiration, $\mathrm{SaO}_{2}$ saturation etc.

\subsection{Cerebral function analysing monitor CFAM}

More than ten years after the CFM was introduced, its design was modified and then implemented in a prototyped cerebral function analysing monitor (CFAM1) (Maynard [11][12]). Initial clinical experiences with the CFAM1 were reported in 1984 (Maynard and Jenkinson [13]). The CFAM1 (® Health care developments Ltd, Herts UK) incorporates a 6808 microprocessor, a twochannel chart recorder and a visual display unit. The CFM trace was modified and in the CFAM1 it gives 90th percentile, the mean and the tenth percentile of the weighted amplitude distribution, with minimum and maximum excursions in every two-second epoch. The CFAM1 incorporates frequency information including beta, alpha, theta and delta activity as well as the percentage of EEG suppression. It computes the relative spectral power of the CFM-transformed EEG signal by means of a modified zero crossing and integration technique. Separate bands for muscle activity and very low frequencies (VLF) are also present.

The CFAM2, released in 1993, can compute two-channel evoked potentials and write two unprocessed EEG signals on a chart recorder. At selected intervals, chart output can be switched between EEG, CFM and spectral signals of either channel. The CFAM2 can be interfaced to an external computer via an RS232C connection.

Very recently a fourth generation CFAM3 (1997) was announced. It provides four channels of evoked potential computation concurrently with up to four channels of EEG monitoring compressed in the conventional CFAM amplitude/frequency distribution format. Data are recorded on the hard disk of a computer and all modes can be output selectively to the thermal chart recorder. The monitor can be trained for research purposes to classify patient data into specific maps, using a neural network system.

Significant improvements were introduced in each of the successive CFAM systems. These improvements were intended to make them suitable for use in many applications, such as monitoring during anaesthesia. However, the data presentation mode, system flexibility and user-friendliness remain rather 
limited.

\section{Description of the MCM}

Because of the limitations of the CFAM systems, we started the development of a new cerebral monitor especially designed for neonatal intensive care. The MCM consists of a two channel EEG-amplifier that is interfaced to a personal computer. The complete system is built onto a trolley to make it mobile. The MCM processes the EEG signals in the amplitude and frequency domains. The major design concepts regarding the processing of the CFM signal have been adopted from the original CFM (Prior [15]).

\subsection{Hardware}

The front-end of the MCM consists of an eight-channel optically isolated referential EEG-amplifier (EEG8) (® Contact Precision Instruments, UK) of which two analogue output signals (channels 1 and 2 ) are connected to an A/Dconverter (® DASH1602, Keithly) located within a Pentium-processor-based personal computer system. For patient safety the computer is powered via a safety transformer. The MCM remotely controls the EEG8 via the I/O control lines of the $A / D$-converter. It can remotely change the amplifier settings (sensitivity, lowpass and highpass cutoff frequency $(-12 \mathrm{~dB} /$ octave) and $50-\mathrm{Hz}$ hum notch filter (50 dB/octave)) and the amplifier mode (calibration, impedance check, unblock). Unblocking is provided to allow re-centring of the signal immediately before a measurement, or at any time when the signal has gone widely out of range, for example, because of a movement artefact. The bandwidth of the EEG8 is from $0.03 \mathrm{~Hz}(\mathrm{HP})$ to $70 \mathrm{~Hz}(\mathrm{LP})$. In calibration mode a $0.5-\mathrm{Hz}$ square-wave calibration signal of fixed amplitude $(100 \mu \mathrm{V})$ is generated and read into the MCM.

\subsection{Data acquisition}

Two analogue EEG signals $\left(P_{3}-F_{z}\right.$ and $\left.P_{4}-F_{z}\right)$ are digitised with a 12-bit resolution. According to the theorem of Shannon, the maximum sampling frequency should be twice the maximum signal frequency, which in this case is about $30 \mathrm{~Hz}$. However, undistorted display of the EEG signals demands a higher frequency; a value of $100 \mathrm{~Hz}$ was chosen. In the MCM the third EEG signal $\left(\mathrm{P}_{3}-\mathrm{P}_{4}\right)$ is computed by subtraction of the two digitised input signals $\left(\left(\mathrm{P}_{3^{-}}\right.\right.$ $\left.\left.\mathrm{F}_{\mathrm{z}}\right)-\left(\mathrm{P}_{4}-\mathrm{F}_{\mathrm{z}}\right)\right)$.

\subsection{Signal processing}

In the MCM, the compressed EEG signal is generated based on the properties of the original CFM/CFAM (Maynard [10][13]). An asymmetric digital bandpass FIR filter is applied to the amplified EEG which flattens the frequency spectrum by giving equal weight to the energy in the signal components between 2 and $15 \mathrm{~Hz}$ (Prior and Maynard [15]). Signals with a frequency below $2 \mathrm{~Hz}$ are reduced to minimise the influence of low frequency artefacts such as movement and/or sweating. High frequency artefacts such as muscle activity measured by electromyography are reduced. The gain-frequency plot of the MCM filter is shown in Figure 3 together with the filter characteristic of the CFM. 
There are only minor differences in the gain-frequency characteristics between 2 and $15 \mathrm{~Hz}$. However, a significant difference exists for frequencies exceeding $15 \mathrm{~Hz}$ : the MCM filter causes an abrupt drop in power, whereas in the CFM the power in the higher frequency components diminishes gradually because of the properties of the analogue filter.

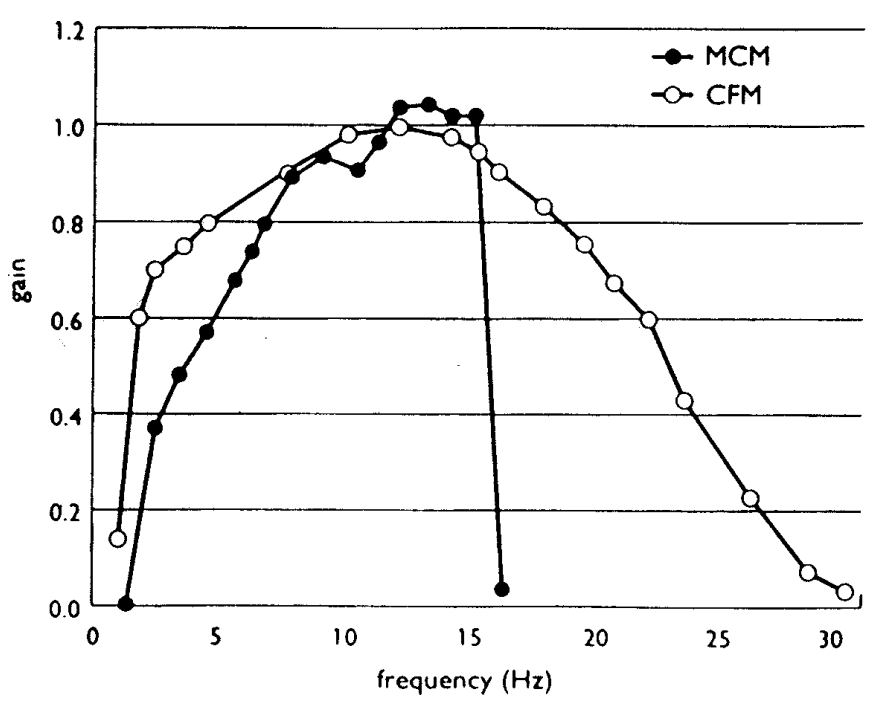

Fig. 3. Bode plot: relative gain as a function of frequency for the original CFM and the MCM.

\subsection{Semi-logarithmic amplitude compression}

So that both very low amplitude EEG and large signals can be displayed without the need for extra gain control and/or range switching, the relation between the input EEG and the compressed output EEG signal has been made linear up to $5 \mu \mathrm{V}$ and semi-logarithmic for larger input values. When the EEG overloads (i.e., signal clipping; EEG amplitude exceeding $200 \mu \mathrm{V}$ ) the CFM amplitude is written above the vertical $y$-axis.

\subsection{Rectification, peak follower and smoothing}

To reduce short-term fluctuations, the semi-logarithmic amplitudecompressed and rectified signal is smoothed by exponential backwards weighting with a time constant of 0.5 seconds (Prior and Maynard [15]). Finally, a so-called peak follower is applied. The output approximates the smooth line drawn through the peaks of the compressed signal with the DC-component retained. 


\section{Properties of Poly}

The MCM system was developed making use of the physiological analysis system Poly ( $₫$ Inspektor Research Systems, Amsterdam), which is a software system for the acquisition, high quality real-time display, on-line analysis and storage of physiological signals. It provides a mouse-driven protocol for quickly setting up, executing and evaluating recordings in particular in an unattended 'monitoring' environment. Real-time alarm detection, manual event marking and review of stored data are all possible during recordings. Figure 4 presents a schematic of the major functions of Poly.

Poly has its own stack oriented programming language called SPL (Signal Processing Language). So-called Measurement Configurations (MCs) can be developed in SPL that describe the signals to be recorded, their calibration, display, processing, alarm detection and analysis. The signals can be just data from external devices like $A / D$ converters but they can also be the results of calculations implemented in SPL code. The program for an MC is split up into three parts: Pre-SPL, Signal-SPL and Alarm-SPL. The Pre-SPL is used for variable declarations and signal-independent initialisations. The Alarm-SPL is used for signal-independent code that, for example, collects results from multiple signals and generates reports. The Signal-SPL code consists of one or -more sections, one for each signal in the MC. Each of these sections produces at least one result, namely the value of the corresponding signal. This value can be automatically displayed and stored to disk.

Each signal or computed variable is connected to a graph. Various screen layouts and special graphs can be defined and configured with simple layout expressions in which original and/or processed signals can be combined. All interactions of the user with the MCM take place via mouse clicks in the different screens and selected keyboard input. It is possible to switch quickly from one pre-defined display configuration screen to another, to add comments and to pause/continue the storage of original and processed data. All input channels in the MCM are sampled with a frequency of $100 \mathrm{~Hz}$. The storage rate of the signals and computed variables is selectable for each signal (up to the maximum sampling frequency).

Poly provides two ways to review recorded data: (i) Data that have been stored during acquisition can be reviewed off-line in a paperless EEG mode. (ii) The original recording or specific parts of it can be marked as a so called 'playback file' and be input to the MCM or some other user-defined Poly program. In this way the original recording can be replayed as if it were being measured at that very moment (Scan mode); all computations and signal manipulations can be carried out again without creating a new recording. Copy \& Process mode is used to create new recordings based on existing ones: they can be used to merge existing recordings with each other or with real-time data, to extract interesting parts and to condense information. 


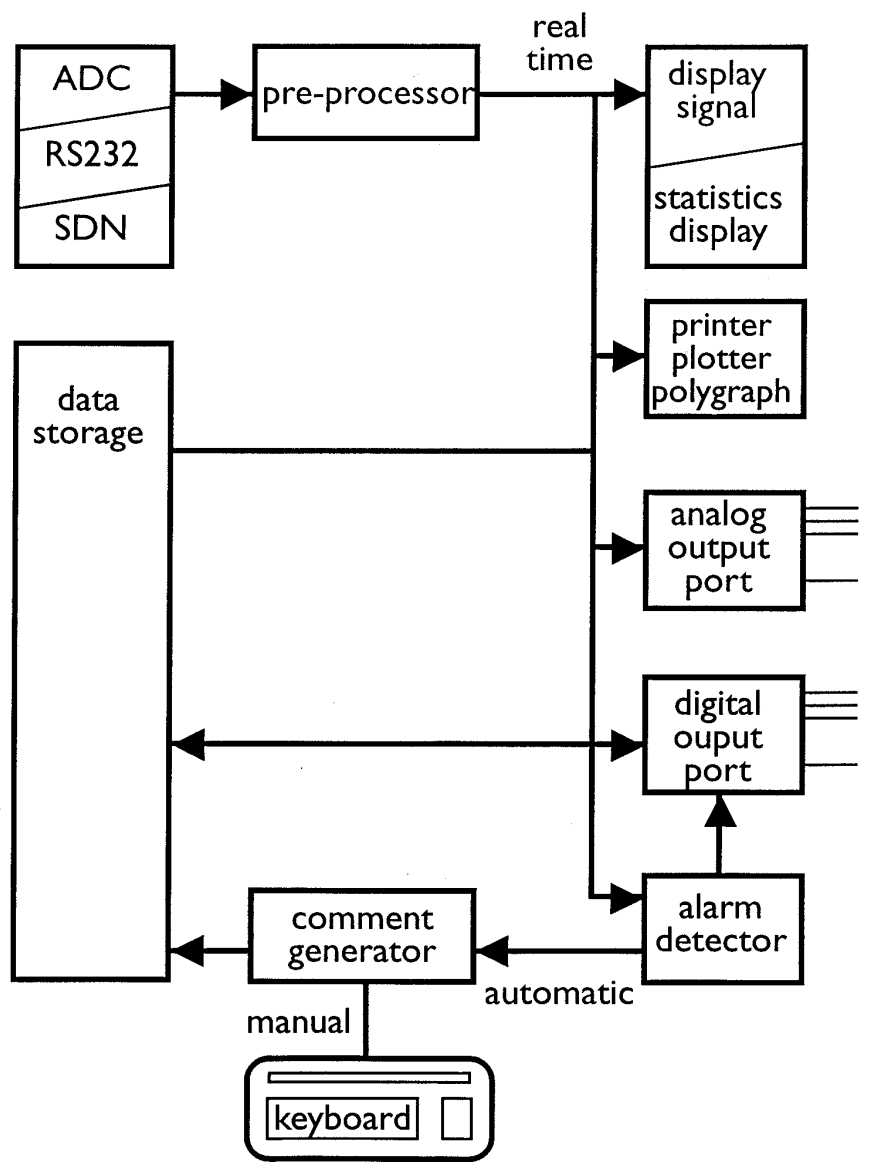

Fig.4. Block diagram of Poly

\section{MCM recording procedure}

\subsection{Calibration}

The MCM starts up by triggering the EEG8 amplifier to generate a $0.5-\mathrm{Hz}$ square-wave calibration signal in both channels. The signals are read into the system and are automatically quantified, after which the values are stored in a calibration file. If the automatic evaluation is incorrect, the values can be adjusted manually.

If the software 'crashes' during monitoring, the MCM system must be quickly restarted. The calibration procedure can be bypassed in that case by retrieving the data from the calibration file. Upon completion of either the full calibration procedure or the retrieval from file the MCM enters monitoring mode.

\subsection{On-line monitoring}

The MCM processes the three EEGs to give several 'Derived Compressed Parameters' (DCPs). The DCPs are: - CFM signal,- absolute and relative 
spectral power in the delta, theta, alpha and beta frequency bands, - absolute spectral band asymmetry (see section Asymmetry) between $P_{3}-F_{z}$ and $P_{4}-F_{z}$, mean and spectral edge frequency (90\% limit) for $P_{3}-F_{z}$ and $P_{4}-F_{z}$,

- percentage of EEG suppression (see section EEG spectral analysis).

The DCPs are computed for each EEG every 3 seconds and stored on hard disk together with the original EEG signals (4.5 Mbytes/hour). The DCPs are Iso stored temporarily in internal memory buffers so that during the ongoingrecording different combinations of original EEG data and DCPs can be presented on the screen. An example is shown in Figure 5.

The sensitivity and time base of the EEGs can be adjusted in on-line mode, all DCPs can be reviewed (backward and forward paging), parameter statistics can be computed and recording comments can be added. The raw EEG data that has already been stored can only be reviewed in off-line review mode. The time base for DCP review is 2700 seconds, i.e., 45 minutes (screen resolution $1280 \mathrm{x}$ 1024 pixels; 900 screen pixels; 1 pixel corresponds with 3 seconds). The minimum and maximum CFM values in every 3-second epoch are calculated and displayed. By drawing a vertical line between the two values, the original penwriting CFM is imitated on the screen. The CFM data are presented on two time bases, either 45 minutes or 3 hours, allowing backward paging per 15 minutes or 1 hour respectively. It is possible to read out CFM amplitudes at any given mouse-selectable time. Furthermore, statistics (mean maximum and mean minimum CFM values; mean EEG amplitude; maximum, minimum, and mean CFM bandwidths; etc.) can be computed over a user selected time interval and stored as a recording comment.

\subsection{Off-line review}

In off-line mode the original EEGs and the DCPs can be reviewed. It is possible to add/edit comments and, guided by these comments, to select relevant data. One or more parts of the original recording can be selected and processed/analysed by a Scan or Copy \& Process MC. Results are generally in the form of automatically generated comments indicating events, report files with statistics and user defined files with derived data such as frequency spectra etc.

Predefined print macros can be used to select documentation and print recorded data. The same method of display expressions is used to define the layout of the printed signals as was used for the recording of the signals.

\section{MCM analysis options}

\subsection{EEG spectral analysis}

The digital filters in the MCM (Williams [22]) are all so-called non-recursive Finite Impulse Response (FIR) filters (Otnes and Enochson [14]), which have stable properties and no phase shift. In Poly, filtering is done through convolution. Each digital filter is a combination of a lowpass and a highpass digital FIR filter: the desired band filter response is described in the frequency domain. This function 
is converted into an impulse response using the inverse Fast Fourier Transform (FFT). This impulse response consists of 257 coefficients and constitutes the socalled filter buffer. Poly computes the convolution of the filter buffer with the current signal buffer. Every sample point in the signal buffer is then shifted one sample (last out, first in).

For every sample point $(10 \mathrm{~ms})$ the absolute value of the filtered signal is computed for each of the three original EEGs in the different frequency bands (delta: 1-4 Hz, theta: 4-8 Hz, alpha: 8-14 Hz, and beta: $14-30 \mathrm{~Hz}$ ). In this way four band-limited signals are generated per EEG channel. The spectral data of each channel in the four frequency bands are stored into a 3-second buffer to facilitate further processing, reduction and presentation of the data. The filtered signals are then rectified and the area under the curve is computed, giving the absolute spectral value for each band for each channel. For each band the relative area with respect to the summed areas of all bands is also computed for a specific signal and expressed as a percentage between 0 and $100 \%$, resulting in relative spectral power.

In both on-line and off-line mode it is possible to compute the mean relative spectral value per band in a user-selected interval as shown in figure 5. Two other informative parameters, namely the mean frequency and the $90 \%$ spectral edge frequency, are computed based on the relative spectral powers for every 3second epoch in each of the four spectral bands. An example is shown in Fig.6. Because relative EEG frequency bands are not independent of each other, the absolute power in each band is also computed and can be shown on the screen.

\subsection{Asymmetry, mean and spectral edge frequency}

Asymmetry is computed so that hemispherical dysfunction and synchrony of maturation EEG can be studied. Asymmetry $\left(A_{i}\right)$ is computed in every 3-second epoch $(\mathrm{k})$ according to

$A_{i}(k)=\left(\left(R_{i}-L_{i}\right) /\left(R_{i}+L_{i}\right)\right) \times 100 \% \quad(i=1,2,3,4)$ for the four spectral bands $(1$ : delta, 2: theta, 3: alpha, 4: beta) . $R_{i}$ is the absolute spectral power of the $i$-th band of the EEG signal $P_{4}-F_{z}$ and $L i$ the corresponding value of $P_{3}-F_{z}$ for a 3-second epoch.

Finally, asymmetry $A_{i}(k)$ is smoothed by exponential backwards averaging using standardised weight factors according to

$A_{i}(k)=0.5 \times A_{i}(k)+0.25 \times A_{i}(k-1)+0.125 \times A_{i}(k-2)+0.0625 \times\left(A_{i}(k-3)+A_{i}(k-\right.$ 4)).

Further parameters derived from the spectral data are the mean and $90 \%$ spectral edge frequencies.

\subsection{Suppression}

To provide an indication of the overall amplitude behaviour of the original EEG, the percentage of the rectified EEG with absolute amplitudes lower than $10 \mu \mathrm{V}$ is computed in every 3 -second epoch. This is called \% suppression. 


\subsection{Extra MCM functions}

\subsubsection{Impedance measurement}

To ensure good quality recordings, the electrode impedances must be attended periodically. The impedance option makes it possible to automatically check the electrode impedances (I-check) at any pre-set and software selectable moment in time. Extra checks can be initiated manually. Whenever an I-check is performed automatically a recording comment is attached to the data file, storing the actual time and the impedance values for later review.

6.4.2. Reliability

Prevention of system failure, or 'crashing', is extremely important for a long-term monitoring system. The number of crashes is reduced because of a built-in 'data wait queue', the filling grade of which is always displayed. If the system becomes overloaded, for example, by keyboard or mouse-click initiated time-consuming actions such as changes in screen display, then the incoming EEG data are temporarily stored in the queue. If the queue overflows, the system crashes. To prevent queue overflow, the system switches to a less time-consuming display when the queue overflow reaches $20 \%$ above the queue capacity.

Another important reliability aspect is to prevent system crashes when there is no more storage capacity on the computer hard disk. In this situation the system automatically stops storing data and gives a warning, but it continues to display new data. Finally, apart from some basic user keys, the keyboard is disabled to minimise the likelihood of unauthorised manipulation of the system. 


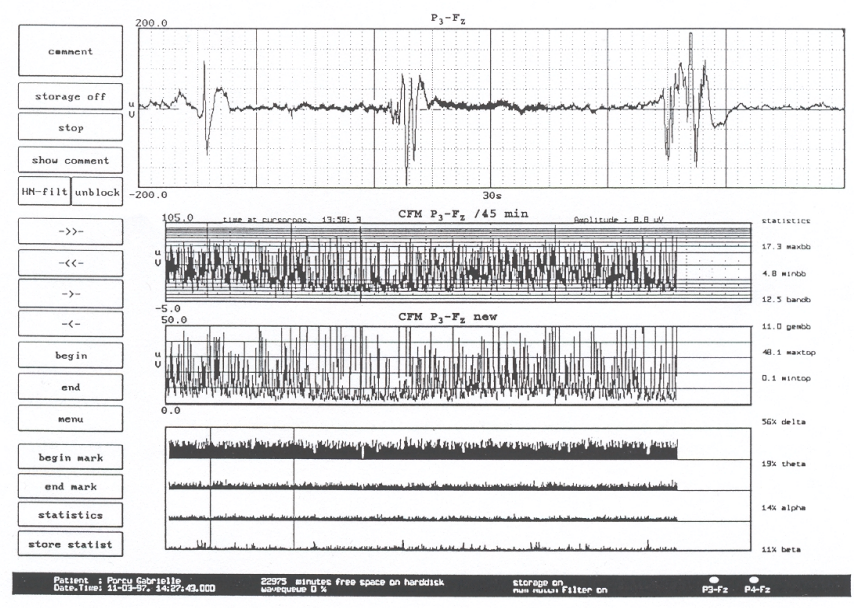

Fig.5. Example of a MCM signal display configuration. The upper window shows a raw EEG signal (time base $30 \mathrm{~s}$ ), the second window the corresponding CFM signal (time base 45 minutes) and the lower window shows the relative spectral powers in the delta, theta, alpha and beta bands (time base 45 minutes). The third window is an alternatively computed CFM signal: vertical lines are from mean value to mean plus standard deviation in every 3 -second epoch.

The various buttons for user interaction are shown at left. Statistics computed for the interval indicated by vertical lines are displayed at the right.

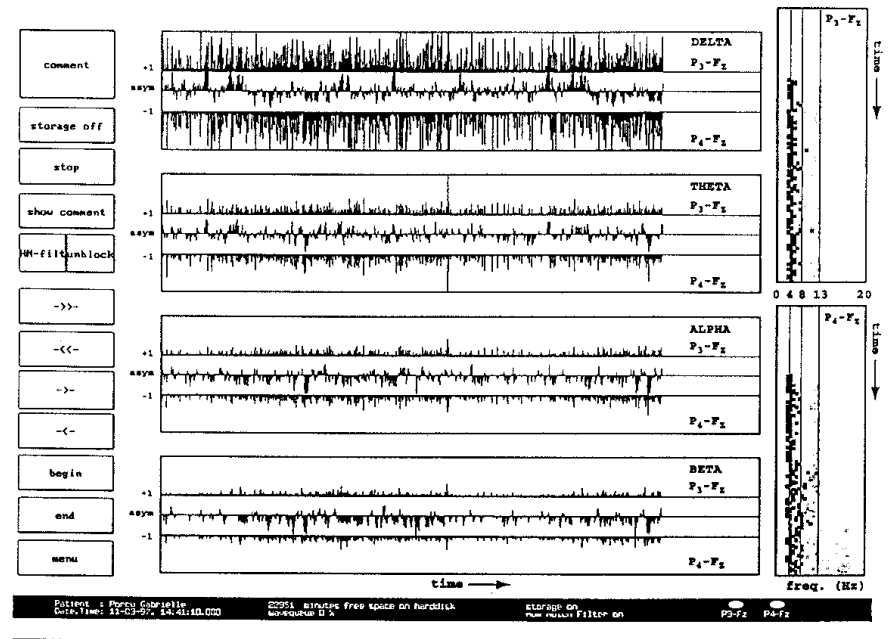

Fig.6. Asymmetry display configuration: absolute spectral powers and their asymmetry (ASYM) in the delta, theta, alpha and beta band (time base 45 minutes). At the right the mean and spectral edge (90\%) frequencies for the $P_{3}-F_{z}$ and $P_{4}-F_{2}$ EEG signals computed for every 3-second epoch are shown in a waterfall diagram. The various buttons for user interaction are shown at the left. 


\section{Discussion}

The MCM system was and is being evaluated in experimental and clinical practice, respectively (Bunt [2]).

Animal experiments performed by our research group showed that isolated severe systemic hypotension affected neuronal activity as reflected by the MCM. A mean arterial pressure of $30 \mathrm{~mm} \mathrm{Hg}$ was the cut-off for cerebral perfusion impairment and neuronal dysfunction in our piglet model (Bunt[2], Gavilanes[4]). An increase of relative delta wave power preceded significant changes in EEG amplitude. We speculate that changes in delta power might offer the possibility to anticipate critical changes in cerebral electrocortical activity and haemodynamics (Gavilanes[4]).

Clinical application so far shows that the equipment does not obstruct the functioning of other systems surrounding the neonate. It fulfils the demands set by the neonatologist and can be applied rapidly by nursing staff. The benefit of the MCM in our NICU setting is its simplicity and ease of interpretation and the need for only minimal attendance over prolonged periods of time. However, the MCM provides a supplementary measure and it cannot replace the conventional EEG. Consequently, a combination of intermittent EEG and continuous MCM is probably optimal to monitor the neonatal brain.

Comparison of the MCM with earlier CFAM systems shows that MCM flexibility and efficiency of data handling and presentation both during on-line recording and off-line review are superior. User interaction is improved for ease of operation.

MCM (asymmetric) digital filtering has several advantages over the analog filtering properties of the original CF(A)M systems; it causes an abrupt instead of a gradual decrease in signal power for signal frequencies exceeding $15 \mathrm{~Hz}$. In this way the EEG is optimally band limited, reducing the influence of aliasing on spectral parameters due to signal frequencies above $15 \mathrm{~Hz}$. Furthermore, the influence of high frequency signals giving rise to artefacts in the EEG is strongly reduced, in particular electromyographic and /or electrocardiographic signals.

MCM spectral analysis is performed on the original EEG and not on the CFM signals, and it applies FFT instead of zero crossing, which makes it more reliable. Finally, not only interdependent relative spectral properties but also absolute spectral properties are computed.

In the MCM various display configurations are implemented which can be paged through both during on-line recording and off-line review. The choice for these types of display was guided by clinical and efficiency arguments. A major design criterion was to display data time compressed together with a zoom function for more detail. Similarly display of combined amplitude and spectral parameters to study their interdependency.

Unlike the CFAM systems, the MCM is not equipped with options for recording evoked potentials. We did not consider this to be an important feature for cerebral NICU monitoring.

On-line simultaneous acquisition of other physiological data, such as blood pressure, heart rate, respiration, eye movements and blood gases may aid in understanding the causes and effects of changes in the EEG. The need to correlate these parameters with MCM parameters has increased. For this reason we plan to 
interface the MCM with the output signals of our standard HP-Merlin monitors.

The CFAM3 offers the possibility to collect data banks on the basis of which EEG can be classified making use of neural network computations. An important aim of ours is to extend the MCM to include algorithms for the detection and classification of the different neonatal EEG patterns such as tracé alternant, burst-suppression, low-voltage EEG and epileptic seizures. 


\section{References}

1. Altafullah, I., Asaikar, S. and Torres, F. (1991) Status epilepticus: clinical experience with two special devices for continuous cerebral monitoring. Acta Neurol Scand, 84, 374-381.

2. Bunt, J., Gavilanes, A., Reulen, J.P.H., Blanco, C. and Vles, J. (1996) The influence of acute hypoxemia and hypovolemic hypotension on neuronal brain activity measured by the cerebral function monitor in newborn piglets. Neuropediatrics, 27, 260-264.

3. Eken, P., Toet, M.C., Groenendaal, F. and de Vries, L.S. (1995) Predictive value of early neuroimaging, pulsed Doppler and neurophysiology in full term infants with hypoxic-ischaemic encephalopathy. Arch. Disease in Childhood, 73(2), F75-80.

4. Gavilanes, A.W.D., Vles, J.H., von Siebenthal, K., Reulen, J.P.H., Nieman, F.H., van Sp[rundel, R. and Blanco, C.E. (1997) Electrocortical brain activity, cerebral haemodynamics and oxygenation during progressive hypotension. Pediatric Research, 41(4,part 2):290A, \#1726.

5. Greisen, G. (1994) Tape-recorded EEG and the cerebral function monitor: amplitude integrated, time-compressed EEG. J. Perinat. Med., 22, 541-546.

6. Hellström-Westas, L., Rósen, I. and Svenningsen, N.W. (1985) Silent seizures in sick infants in early life. Acta Paediatr. Scand., 74, 741-748.

7. Hellström-Westas, L. (1992) Comparison between tape-recorded and amplitudeintegrated EEG monitoring in sick newborn infants. Acta Paediatr., 81, 812-819

8. Hellström-Westas, L., Rósen, I. and NW Svenningsen, N.W. (1995) Predictive value of early continuous amplitude integrated EEG recordings on outcome after severe birth asphyxia in full term infants. Arch. Disease in Childhood, 72, F34-38. 9. Holmes, G., Rowe, J., Hafford, J., Schmidt, R., Testa, M. and Zimmerman, A. (1982) Prognostic value of the electroencephalogram in neonatal asphyxia. Electroencephalogr. Clin. Neurophysiol., 53, 60-72

10. Maynard, D.E., Prior, P.F. and Scott, D.F. (1969) Device for continuous monitoring of cerebral activity in resuscitated patients. Brit. Med. J., 4, 545-546.

11. Maynard, D.E. (1977). The cerebral function analysing monitor (CFAM). Electroencephalogr. Clin. Neurophysiol., 43, 479.

12. Maynard, D.E. (1979) Development of the CFM: the cerebral function analysing monitor (CFAM). Annales de L'Anesthesiologie Francaise, 20, 253-255.

13. Maynard, D.E. and Jenkinson, J.L. (1984) The cerebral function analysing monitor. Initial clinical experience, application and further development. Anaesthesia, 39, 678-690.

14. Otnes, R.K. and Enochson, L. (1978). Applied Time series analysis. Vol.1. Basic techniques. John Wiley \& Sons, chapter 4.12, nonrecursive (FIR) filters.

15. Prior, P.F. and Maynard, D.E. (1986) Monitoring cerebral function. Long term monitoring of EEG and Evoked Potentials. Elsevier Science Publishers, Amsterdam.

16. Procaccio, F., Menasce, G., Sacchi, L. and Boselli, L. (1991) Effects of Thiopentone and mannitol on cerebral perfusion pressure and EEG in head injured patients with intracranial hypertension. Agressologie, 32, 8-9, 381-385. 
17. Thornberg, E. and Ekström-Jodal, B. (1994). Cerebral function monitoring: a method of predicting outcome in term neonates after severe perinatal asphyxia. Acta Paediatr., 83, 596-601.

18. Thornberg, E. and Thiringer, K. (1990) Normal pattern of the cerebral function monitor trace in term and preterm neonates. Acta Paediatr Scand., 79, 20-22.

19. Thomsen, C.E. and Prior, P.F. (1996) Quantitative EEG in assessment of anaesthetic depth: comparative study of methodology. Brit. J. of Anaesthesia, 77, 172-178.

20. Toner, C.C., Underwood, M.J., Wright, S.J., Cooper, G.J., Reynolds, P. and Withington, P.S. (1995) The cerebral function monitor (CFM) is a useful addition to a bilateral carotid artery, vein graft model. Laboratory Animals, 29, 427-431

21. Viniker, D.A., Maynard, D.E. and Scott, D.F. (1984). Cerebral function monitor studies in neonates. Clin. Electroencephalogr., 15, 185-92.

22. Williams, C.S. (1986) Designing Digital Filters. Prentice Hall information and system sciences series. Englewood Cliffs, New Jersey. 
CHAPTER 4.

EFFECT OF REPETITIVE UMBILICAL CORD OCCLUSIONS ON NEURONAL BRAIN ACTIVITY MEASURED BY THE CEREBRAL FUNCTION ANALYZING MONITOR AND HISTOLOGIC OUTCOME IN IMMATURE FETAL SHEEP

H. Keunen, J.S.H. Vles, J.L.H. Van Reempts, A.W.D.Gavilanes and T.H.M. Hasaart

J Soc Gynecol Investig 2000; 7(4): 218-23 


\section{Abstract}

Objective: To examine the effect of repetitive total umbilical cord occlusions on electrocortical brain activity as measured by cerebral function analyzing monitoring (CAFM) and the histologic outcome in immature sheep fetuses.

Study Design: We performed brief repeated total umbilical cord occlusions, two every 5 minutes, in 12 immature sheep fetuses (at 90 days of gestation, term 147 days) until fetal mean arterial pressure dropped below $50 \%$ of baseline value during two successive occlusions. A pair of electrodes was inserted on the parietal dura for recording of electrocortical brain activity (ECoG). Off-line ECoG signal processing consisted of amplitude integrated analysis (CFAM) and spectral analysis. Fetal blood gas analyses were performed at regular intervals just before subsequent umbilical cord occlusions. Three days after the occlusion neuronal damage was evaluated histologically in three regions of the fetal brain.

Results: CFAM amplitide parameters decreased significantly during the first occlusion and remained so during the entire repetitive occlusion period (analysis of variance $[A N O V A] ; P<.05)$. Spectral analysis of the ECoG signal demonstrated no changes in the distribution of frequency bands. Progressive acidemia and hypotension developed with ongoing occlusions. Five fetuses died at the end or shortly after the entire repetitive occlusion period. No neuronal damage or macroscopic intraventricular and/or germinal matrix hemorrhage was observed in the surviving fetuses.

Conclusion: Repetitive umbilical cord occlusions in immature sheep fetuses resulted in functional, not structural changes of the fetal brain in surviving fetuses. At this gestational age, amplitude analysis is more sensitive than spectral analysis of the ECoG signal to functional changes of the compromised fetal brain.

Author Keywords: Sheep; fetus; asphyxia; CFAM; brain

\section{Introduction}

Recently, we reported the absence of neuronal damage in immature fetal sheep after a single period of total umbilical cord occlusion up to 20 minutes.[1] In clinical practice, asphyxia during labor is often characterized by short repetitive periods of fetal compromise. The question remains whether neuronal damage can be observed when immature sheep fetuses are subjected to repetitive periods of umbilical cord occlusions rather than a single transient occlusion.

Several methods have been used to monitor the effect of asphyxia on the fetal sheep brain. These methods include the registration of raw electrocorticogram signals (ECoG), cortical impedance, and sensory and auditory evoked potentials. Another possible method is the cerebral function analyzing monitor (CFAM).[2] The CFAM offers a one-channel registration of cerebral activity from two biparietal electrodes and is represented as a band. The CFAM is a system of amplitude integrated electroencephalograms which can be used to predict gestational age and neurologic outcome of the asphyxiated newborn. [3] The use of CFAM as an on-line monitor might offer the possibility to monitor cortical brain activity during periods of umbilical cord occlusion. 
In this study we tested the hypothesis that repetitive total umbilical cord occlusions result in neuronal damage in immature fetal sheep. Furthermore, we introduced the CFAM in this chronic fetal sheep preparation to evaluate its ability to monitor periodic changes in electrocortical activity during repetitive periods of fetal compromise. Therefore, we subjected immature sheep fetuses to asphyxia induced by repetitive umbilical cord occlusions to examine the neuronal outcome 3 days after the insult, the changes in electrocortical activity during the occlusions, and their possible interrelationships.

\section{Materials and methods}

\section{Surgery}

Twelve immature fetal sheep (gestational age: mean 90.6 days; range 87-93 days) underwent surgery using sterile techniques under general anesthesia (sodium thiopentone $1 \mathrm{~g} / 70 \mathrm{~kg}$ intravenously for induction, $1 \%$ halothane in a 2:1 nitrous oxide and oxygen mixture for maintenance). We performed a midline abdominal incision. We incised the uterus and partly exteriorized the fetus to allow access to the umbilical cord. A reversible cord occluder (Model VO-4; Rhodes Medical Instruments, Woodland Hills, CA) was placed around the umbilical cord and then tested for total occlusion volume by infusing sterile saline. We inserted a polyvinyl catheter $(0.5 \mathrm{~mm}$ inner diameter, $1.0 \mathrm{~mm}$ outer diameter) in the carotid artery and directed it toward the heart to measure fetal arterial blood pressure (MAP) and heart rate and allow arterial blood sampling. The dead-volume space of the catheter was filled with heparin $(5000 \mathrm{IU} / \mathrm{mL})$ which remained in situ and was removed on the day of the experiment. For registration of ECoG a pair of shielded stainless-steel electrodes was inserted through biparietal drilled burr holes on the parietal dura. A reference electrode was sewn subcutaneously into the scalp. We exteriorized all catheters and electrodes through a small incision in the flank of the ewe. Ewes were housed in individual cages with free access to food and water. The ewes were allowed a recovery period of at least 3 days after the operation before experiments were started. Guidelines for care and use of animals, as approved by the local animal medical ethics committee, were followed.

\section{Measurements}

Fetal blood pressure was corrected for amniotic pressure. We derived the fetal heart rate from the blood pressure waveform. Signals were collected on a personal computer, using a hemodynamic data acquisition system custom-built by the Instrumental Department of the University of Maastricht. Beat-by-beat measurements of fetal heart rate and mean arterial pressure were displayed on-line on a monitor. We measured fetal blood gas values and $\mathrm{pH}$ with an automated analyzer (AVL 993; AVL Medical Instruments AG, Schaffhausen, Switzerland) and corrected them for fetal temperature $(T=39.5 \mathrm{C})$. Fetal hemoglobin concentration $(\mathrm{mmol} / \mathrm{L})$ and hemoglobin oxygen saturation (percentage) were measured with a hemoximeter (OSM2 hemoximeter; Radiometer, Copenhagen, Denmark). Arterial oxygen content was calculated as follows: arterial oxygen content $(\mathrm{mL} / \mathrm{dL})=1.36 \times$ hemoglobin concentration $(\mathrm{mmol} / \mathrm{L}) \times$ hemoglobin oxygen saturation $(\%) \times 1.611$.[4]

\section{Experiments}

We started experiments when sheep were at a mean gestational age of 93.6 days 
(range 90-96). Fetal heart rate and blood pressure were monitored continuously. We performed baseline fetal arterial blood gas analyses 30 and 5 minutes before the first umbilical cord occlusion and immediately before every fourth occlusion. Total umbilical cord occlusion was achieved by infusing sterile saline with the predetermined occlusion volume into the occluder. The umbilical cord was repetitively occluded for 2 of every 5 minutes. Repetitive occlusions were maintained until the fetal MAP decreased below $50 \%$ of baseline value during two successive occlusions. To limit excessive blood sampling, the maximum time allowed to reach this degree of hypotension during the repetitive occlusion period was 4 hours.

Off-line ECoG signal processing

Off-line processing of the raw ECoG signal included weighted digital band filtering between 2 and $15 \mathrm{~Hz}$, rectification, logarithmic amplitude compression, and smoothing of the signal. For further analysis epochs of the CFAM signal lasting 3.6 seconds were selected. We calculated CFAM parameters (mean amplitude distribution and bandwidth in microvolts) using the software program Poly (Inspector Research System BV, Amsterdam, The Netherlands), modified by the Department of Clinical Neurophysiology, University Hospital Maastricht. Two epochs of 10 minutes were used to calculate baseline CFAM values. CFAM parameters during occlusions were obtained at the first and last occlusion, and at the occlusion halfway the entire repetitive occlusion period (eg, at the 12th occlusion when a total of 24 occlusions were administered). At every time point one epoch of 3 minutes before and after an occlusion and an epoch during the 2-minute occlusion were calculated. In addition, the ECoG of the similar epochs was subjected to spectral analysis by means of Fourier transformation resulting in a percent distribution of the raw ECoG signal over the following frequency bands: delta (1 to $4 \mathrm{~Hz}$ ), theta (4 to $8 \mathrm{~Hz}$ ), alpha (8 to $14 \mathrm{~Hz}$ ), and beta (14 to $30 \mathrm{~Hz}$ ).

Fixation and histologic evaluation

Three days after the cord occlusion, a second laparotomy of the ewe was performed under general anesthesia (sodium thiopentone $1 \mathrm{~g} / 70 \mathrm{~kg}$ intravenously for induction, $1 \%$ halothane in a 2:1 nitrous oxide and oxygen mixture for maintenance). The fetal heart was approached by median thoracotomy. We heparinized the fetus with an injection of 20,000 U heparin into the left cardiac ventricle. A blunt steel catheter was inserted in the left cardiac ventricle. The right atrium was opened and both external jugular veins were dissected and cut. Five hundred milliliters of isotonic and isocolloidal fluid (Haemaccel; Baxter BV, Utrecht, The Netherlands) was infused in the left ventricle followed by $750 \mathrm{~mL}$ Karnovsky's fixative ( $2 \%$ formaldehyde and $2.5 \%$ glutaraldehyde in phosphate buffer $0.1 \mathrm{~mol} / \mathrm{L}$, $\mathrm{pH}$ 7.40). After decapitation the skull was opened and the head was submerged into the same fixative for additional immersion fixation. After 1 day of immersion fixation we removed the fetal brain from the skull for histologic evaluation. Vibratome sections $(200 \mu \mathrm{m})$ were cut from three areas: first, the parasagittal cortex, defined as the gyrus adjacent to the sagittal sulcus; second, the dorsal gyrus of the hippocampus; and third, the cerebellum. Sections were postfixed in $2 \%$ osmium tetroxide, buffered with uranyl acetate $(0.05 \mathrm{~mol} / \mathrm{L}, \mathrm{pH} 7.40)$, dehydrated in graded series of ethanol, and routinely imbedded in Epon. Light-microscopic 
evaluation was performed on $2 \mu \mathrm{m}$ sections stained with Toluidine blue by an observer (JVR) unaware of the experimental situation. Neuronal damage was defined as the degree of coagulative cell change per high-power field, graded on a nine-point scale in which $0=$ absence of coagulative changes and $4=100 \%$ of the tissue damaged. $[1, \underline{5}$ and $\underline{6}]$ Specific interest was directed toward the neuronal cells in the cortex, the pyramidal neurons in the CA1 and CA2 sector of the hippocampus, and the Purkinje cells in the cerebellum.

Statistics

Data are presented as mean \pm standard error of the mean. We compared blood gas and acid base status analyses, hemodynamic parameters, CFAM amplitude parameters, and the results of spectral analysis by analysis of variance (ANOVA). We compared changes relative to baseline with t tests with Tukey's correction for multiple comparisons. Repeated measures were used when appropriate. $\mathrm{P}<.05$ was considered.

\section{Results}

The experimental group consisted of 12 fetal lambs with a mean gestational age of 93.2 days (range 90-96) and weight of $716 \mathrm{~g}$ (range 608-894). The fetuses were subjected to a total of 34 occlusions (range 21-48). Of 12 fetuses, five died at the end or shortly after the occlusion period. Table 1 describes the results of the blood gas and acid-base status analyses at baseline, just before the first, middle, and final occlusion, and 2 hours after the final occlusion. Repetitive umbilical cord occlusions resulted in moderate to severe acidosis, mild hypercapnia, and moderate hypoxia. In the surviving fetuses all parameters returned to baseline values within 2 hours after the final occlusion.

The results of the fetal MAP and heart rate measurements at similar time points are depicted in Table 2. Repetitive umbilical cord occlusions resulted in repetitive periods of bradycardia. During occlusions, fetal MAP demonstrated a biphasic response, which consisted of an initial increase followed by a subsequent decrease resulting in severe hypotension during the final occlusions. Likewise, between occlusions fetal MAP first increased and then decreased to values below but not significantly different from baseline values. Nonsurviving fetuses failed to recover after release of the last occlusion. They remained bradycardic and hypotensive until they died.

The results of the off-line CFAM amplitude and spectral analysis of the ECoG are depicted in Figure 1 and Figure 2. All CFAM amplitude parameters demonstrated a significant decrease during the entire repetitive occlusion period compared with baseline (ANOVA; $P<.05)$. A decrease in all CFAM amplitude parameters except CFAM width was observed during the first occlusion and remained depressed throughout the entire repetitive occlusion period. Of the surviving fetuses, all CFAM amplitude parameters recovered to baseline values at 2 hours after the final occlusion. Spectral analysis of the ECoG signal demonstrated no differences between the baseline and entire repetitive occlusion period. 
Macroscopic and microscopic evaluation of brain damage were performed only on surviving fetuses. Macroscopic inspection showed no evidence for intraventriculargerminal matrix hemorrhage (IVH-GMH). Good fixation quality was demonstrated by the absence of residual erythrocytes in open microvessels and the absence of disseminated cell swelling. Light-microscopic evaluation of the surviving fetuses did not demonstrate neuronal damage in the parasagittal cortex, hippocampus, and cerebellum (score for all surviving fetuses $=0$ ).

Table 1.

Time Course of Blood Gas and Acid-Base Status Analysis

\begin{tabular}{lcccc}
\hline \multicolumn{1}{c}{ Period } & $\mathrm{pH}$ & $\mathrm{PaCO}_{2}(\mathrm{kPa})$ & $\mathrm{CaO}_{2}(\mathrm{mmol} / \mathrm{L})$ & Base excess $(\mathrm{mmol} / \mathrm{L})$ \\
\hline Baseline & $7.34 \pm 0.01$ & $5.79 \pm 0.13$ & $5.43 \pm 0.42$ & $-1.48 \pm 0.59$ \\
Occlusion & & & & \\
First & $7.33 \pm 0.01$ & $5.70 \pm 0.14$ & $6.20 \pm 0.33$ & $-2.26 \pm 0.54$ \\
Middle & $7.17 \pm 0.02^{*}$ & $6.55 \pm 0.22^{*}$ & $6.25 \pm 0.28$ & $-10.64 \pm 0.67^{*}$ \\
Last & $7.09 \pm 0.02^{*}$ & $7.43 \pm 0.41^{*}$ & $4.03 \pm 0.46^{*}$ & $-13.15 \pm 0.90^{*}$ \\
& $7.32 \pm 0.02$ & $6.01 \pm 0.17$ & $5.24 \pm 0.35$ & $-2.09 \pm 0.91$ \\
\hline
\end{tabular}

Fetal arterial blood samples were taken at baseline, just before the first, middle, and last occlusion (all fetuses, $n=12$ ), and 2 hours after the entire repetitive occlusion period (surviving fetuses, $n=7$ ). $\mathrm{P}<.05$, $\mathrm{t}$ test, compared with baseline.

Table 2.

Time Course of Mean Fetal Heart Rate During Baseline and $2 \mathrm{~h}$ After the Entire Repetitive Occlusion Period, and Minimum Fetal Heart Rate During the First, Middle, and Last Occlusion (beats per minute)

\begin{tabular}{lcccc}
\hline \multicolumn{1}{c}{ Period } & FHR & MAP max & MAP min & MAP int \\
\hline Baseline & $197.33 \pm 4.23$ & & & $32.73 \pm 1.40$ \\
Occlusion & & & & \\
First & $68.11 \pm 3.15^{*}$ & $48.41 \pm 1.84^{*}$ & $30.21 \pm 1.94$ & $44.04 \pm 1.72^{*}$ \\
Middle & $86.76 \pm 3.08^{*}$ & $45.27 \pm 2.49^{*}$ & $28.83 \pm 1.51$ & $38.33 \pm 2.02^{*}$ \\
Last & $72.95 \pm 3.13^{*}$ & $38.21 \pm 2.37^{*}$ & $13.08 \pm 1.90^{*}$ & $30.92 \pm 1.87$ \\
& $191.11 \pm 3.82$ & & & $29.54 \pm 2.23$ \\
\hline
\end{tabular}

Time course of the maximum (MAP max) and minimum (MAP min) fetal mean arterial pressure during the first, middle, and last occlusion is shown, as well as time course of the fetal mean arterial pressure during baseline and 2 hours after the entire repetitive occlusion period, and between occlusions after the first and middle and just before the last occlusion (MAP int) (mmHg). "P $<.05$, $t$ test, compared with baseline. 
Figure 1.

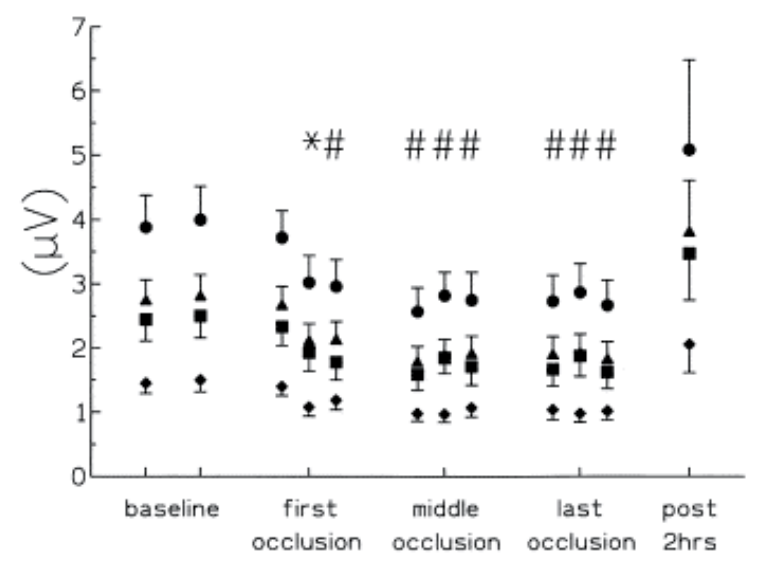

Time course of CFAM ( $\mu \mathrm{V}$, CFAM maximum (closed circles) CFAM minimum (closed diamonds), CFAM width (closed squares), and CFAM (closed triangles) during baseline (two epochs of 10 minutes), first, middle, and last occlusion (three epochs: one epoch of 3 minutes before and after, and one epoch during the occlusion, all fetuses, $\mathrm{n}=12$ ), and 2 hours after the entire repetitive occlusion period (one epoch of 10 minutes, $>2$ hours, only surviving fetuses, $n=7$ ). ANOVA: $P<.05,{ }^{\#} P<.05$, $t$ test, compared with baseline, all CFAM parameters. ${ }^{*} P<.05, t$ test, compared with baseline, all CFAM parameters except CFAM width. Values are mean \pm SEM.

Figure 2.

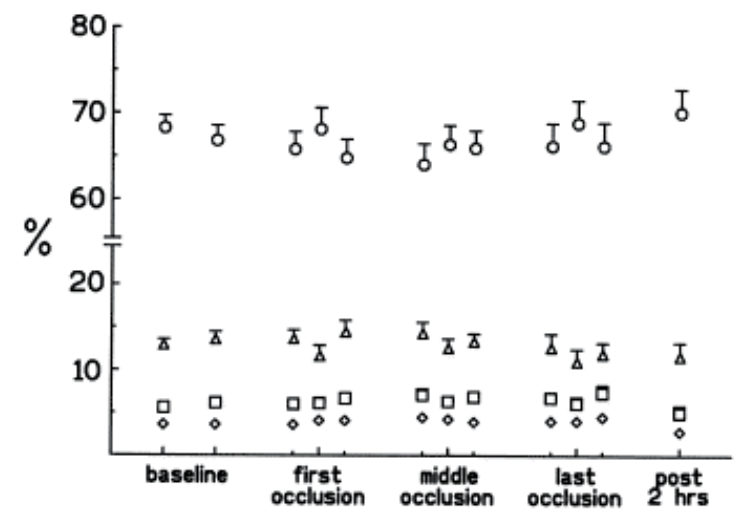

Time course of spectral analysis of the ECoG signal (\%, delta band (open circles), theta band (open triangles), alpha band (open squares), and beta band (open diamonds) during baseline (two epochs of 10 minutes), first, middle, and last occlusion (three epochs: one epoch of 3 minutes before and after, and one epoch during the occlusion, all fetuses, $n=12$ ), and 2 hours after the entire repetitive occlusion period (one epoch of 10 minutes, $>2$ hours only surviving fetuses, $n=7$ ). ANOVA: $P>.05$. 


\section{Discussion}

Repetitive umbilical cord occlusions in immature fetal sheep in this experimental setup resulted in repetitive periods of hypertension and hypotension, bradycardia, and sustained mixed acidosis. The degree of hypotension was comparable to the changes in MAP observed in our previous study where immature fetuses were subjected to a single total umbilical cord occlusion up to 20 minutes.[1] In concordance with repetitive umbilical cord occlusions in mature fetal lambs, changes in fetal MAP demonstrated a biphasic response which consisted of an initial rise in MAP followed by a subsequent decrease resulting in severe hypotension during the final occlusions. [7] Because arterial blood pressure is the result of the product of cardiac output times peripheral vascular resistance, hypotension at the end of the repetitive occlusion period may result in a decrease of one or both of these parameters. Severe hypoxia and depletion of myocardial glycogen stores may decrease cardiac output. Furthermore, in fetal lambs a decrease in total peripheral vascular resistance was observed during severe asphyxia. [8] Whether a decrease in cardiac output or peripheral vascular resistance was responsible for the repetitive periods of hypotension observed in this study remains unsolved.

The absence of neuronal damage and IVH-GMHs agrees with our previous observations in immature fetal lambs after a single umbilical cord occlusion up to 20 minutes.[1] The degree of hypotension at the end of the final occlusions in this study was comparable to the changes of MAP observed in the transient total umbilical cord occlusion. However, acidosis in the single transient occlusion group was more severe. In part, this could be explained by the timing of the fetal blood gas analysis, which was performed just before an occlusion in the present study compared with fetal blood gas analysis at the end of the single umbilical occlusion period. [1]

Repetitive umbilical cord occlusions in mature sheep fetuses resulted in cerebral damage ranging from mild selective neuronal loss to focal infarcts in the parasagittal cortex, thalamus, and cerebellum.[9] This ontogenetic difference in neuronal damage also was observed in a comparative study in which mature and immature fetal lambs were subjected to 10 minutes' total umbilical cord occlusion. [10] Considering the mortality rate in the present study, fetal demise probably occurred before neuronal damage could develop. However, histologic analysis was not performed on nonsurviving fetuses for obvious reasons of postmortem changes. Several factors may be involved in the apparent resistance to neuronal damage of the immature brain after asphyxia. The immature brain has small and less branched neurons with fewer synapses and therefore low basal energy demands. Consequently, immature fetal sheep have a low cerebral metabolism. [11] In combination with preservation of cerebral circulation, energy metabolism can be maintained for a long period during asphyxia. [12] Brain damage may be dependent on mechanisms other than a lower metabolic rate. In vitro studies in hippocampal slices demonstrated lower postischemic inhibition of protein synthesis during ischemic circumstances in slices of immature fetal guinee pigs compared with mature fetuses. [13] These postischemic disturbances in protein synthesis reflect the ontogenic changes in vulnerability of the brain. Likewise, ontogenic factors regarding both excitatory and inhibitory neurotransmitters may have a profound 
effect on the age related susceptibility to hypoxic-ischemic brain injury. [14, $\underline{15}$ and 16]

Although asphyxia and arterial pressure fluctuations were present, IVH-GMHs were absent. So far, one study succeeded in producing IVH-GMHs in fetal lambs, although under acute experimental circumstances.[17] However, several studies failed to demonstrate similar results in chronic fetal sheep preparations. Intact cerebral autoregulation was described in immature fetal lambs. [18] It is known that asphyxia can abolish the protective mechanism of autoregulation. [19] Likely, in the present study hypertensive periods during the first occlusions were not coincidental with severe asphyxia, whereas hypotension during the final occlusions was not severe enough to decrease below the lower limit of cerebral vascular autoregulation.

To date, this is the first report on the use of CFAM in the chronic fetal sheep preparation. As a simplified one-channel electroencephalogram registration the CFAM allows noninvasive long-term recording of cortical activity and similtaneous rejection of artifacts. At present it is used in neonatal intensive care units for the monitoring of seizures. Furthermore, it is suggested that CFAM can be used as a prognostic tool after severe peripartal asphyxia.[3, $\underline{20}$ and 21] Currently, a newly designed computerized cerebral function monitor is in use at our neonatal intensive care unit. [22]

Fetal CFAM amplitude parameters decreased during the repetitive occlusion period. However, this decrease already occurred during the first occlusion and remained so throughout the entire repetitive occlusion period. Evidently, an umbilical occlusion of 2 minutes resulted in an impairment of cortical activity, which remained depressed throughout the following 3-minute recovery period and subsequent occlusions. No further decrease in CFAM amplitude parameters was observed during the time course of repetitive cord occlusions. At 2 hours after the final occlusion, CFAM amplitude parameters recovered to baseline recordings in the surviving fetuses.

Evidence of seizure activity, periodic increases in CFAM amplitude parameters and fetal MAP, was not observed in the recovery period. The rapid decrease in ECoG activity also was observed in intermittent umbilical cord occlusions in mature fetal lambs.[9] However, in that study epileptiform ECoG changes were observed in the recovery period which were coincidental with subsequent neuronal damage in the parasagital cortex. The rapid decrease in CFAM amplitude parameters is not in concert with previous observations in an acute experimental setup in newborn piglets. [23] In that study, a decrease in CFAM amplitude parameters was observed only when the piglets were subjected to isolated hypovolemic hypotension or a combination of hypotension and hypoxemia. Isolated hypoxemia did not result in a decrease of the CFAM amplitude parameters. In the present study, early occlusions may result in hypoxemia, not hypotension. Furthermore, final occlusions resulted in a combination of hypoxemia and hypotension. Therefore, we expected a gradual decrease in CFAM amplitude parameters toward the final occlusions in the present investigation. Apparently, repetitive occlusions did result in impairment of neuronal activity based on hypoxemia in early occlusions and a combination of hypoxemia and hypotension during final occlusions. Spectral analysis demonstrated no shifts in the distribution of the frequency bands. In a severely compromised brain, a shift to lower frequencies might be expected. Consequently, the insults implied in this study 
were not severe enough to change electrocortical brain activity in this way. On the other hand, electrocortical activity alters as a function of gestational age. [24] A transition from disorganized to cyclic electrocortical activity occurs at a gestational age of approximately 115-120 days. The maturational changes consist primarily of a shift of the distribution of frequency bands from the lower delta to a higher frequency band beta. Therefore, a shift toward lower frequencies bands during asphyxia might not have been detectable as a result of the already predominant lower frequencies in the electrocorticogram of the immature fetuses in the present study.

In conclusion, repetitive umbilical cord occlusion resulted in asphyxia and ultimately in repetitive periods of hypotension. However, we did not observe neuronal damage. CFAM in the fetal preparation is feasible. CFAM amplitude parameters decreased during the first occlusions and remained so throughout the entire repetitive occlusion period. On the other hand, spectral analysis of the EcoG signal did not demonstrate any changes during the entire repetitive occlusion period. Therefore, in the present study, functional changes in the fetal brain were amplitude rather than frequency related. Further investigations are warranted to study the relationship between CFAM amplitude parameters and hemodynamic and histologic changes during and after fetal compromise at various gestational ages. 


\section{References}

1. H. Keunen, C.E. Blanco, J.L.H. Van Reempts and T.H.M. Hasaart, Absence of neuronal damage after total umbilical cord occlusion of 10, 15 and 20 minutes in midgestation fetal sheep. Am J Obstet Gynecol 176 (1997), pp. 515-520

2. D.E. Maynard, Development of the CFM: The cerebral function analysing monitor. Ann Anesth Franc 3 (1979), p. 253

3. L. Hellstrom-Westas, I. Rosen and N.W. Svenningsen, Predictive value of early continuous amplitude integrated EEG recordings on outcome after severe birth asphyxia in full term infants. Arch Dis Child72 (1995), pp. F34-F38.

4. M.A.H.B.M. van der Hoeven, W.J. Maertzdorf and C.E. Blanco, Mixed venous oxygen saturation $\left(\mathrm{SvO}_{2}\right)$ and biochemical parameters of hypoxia during progressive hypoxemia in 10-14 day old piglets. Pediatr Res 42 (1997), pp. 878-884.

5. J.L.H. Van Reempts, The hypoxic brain: Histological and ultrastructural aspects. Behav Brain Res 14 (1984), pp. 99-108

6. J.L.H. Van Reempts and M. Borgers, Structural damage in experimental cerebral ischemia. In: A. Schurr and B.M. Rigor, Editors, Cerebral ischemia and resuscitation, CRC Press, Boca Raton, FL (1990), pp. 235-257.

7. H.H. de Haan, A.J. Gunn and P.D. Gluckman, Fetal heart rate changes do not reflect cardiovascular deterioration during brief repeated umbilical cord occlusions in near-term fetal lambs. Am J Obstet Gynecol 176 (1997), pp. 8-17.

8. A. Jensen, H. Gips, M. Hohmann and W. Künzel, Adrenal endocrine and circulatory responses to acute prolonged asphyxia in surviving and non-surviving fetal sheep near term. In: W. Künzel and A. Jensen, Editors, The endocrine control of the fetus, Springer Verlag, Berlin (1988), pp. 64-79.

9. H.H. de Haan, A.J. Gunn, C.E. Williams and P.D. Gluckman, Brief repeated umbilical cord occlusions cause sustained cytotoxic cerebral edema and focal infarcts in near-term fetal lambs. Pediatr Res 41 (1997), pp. 96- 104.

10. E.C. Mallard, A.J. Gunn, C.E. Williams, B.M. Johnston and P.D. Gluckman, Increased vulnerability to neuronal damage after umbilical cord occlusion in fetal sheep with advancing gestation. Am J Obstet Gynecol 170 (1994), pp. 206-214

11. C.A. Gleason, C. Hamm and M.D. Jones, Jr, Cerebral blood flow, oxygenation, and carbohydrate metabolism in immature fetal sheep in utero. Am J Physiol 256 (1989), pp. R1264-R1268

12. C.A. Gleason, C. Hamm and M.D. Jones, Jr, Effect of acute hypoxemia on brain blood flow and oxygen metabolism in immature fetal sheep. Am J Physiol 258 (1990), pp. H1064-H1069.

13. R. Berger, B. Djuricic, A. Jensen, K.A. Hossmann and W. Paschen, Ontogenic differences in energy metabolism and inhibition of protein synthesis in hippocampal slices during in vitro ischemia and 24h of recovery. Dev Brain Res 91 (1995), pp. 281-291.

14. J.W. MacDonald, F.S. Silverstein and M.V. Johnston, Neurotoxicity of N-methyl$\mathrm{D}$-aspartate is markedly enhanced in the developing rat central nervous system. Brain Res 459 (1988), pp. 200-203. 
15. S. Thurber, M.A. Mikati, C.E. Stafstrom, F.E. Jensen and G.L. Holmes, Quisqualic acid-induced seizures during development: A behavioral and EEG study. Epilepsia 35 (1994), pp. 868-875.

16. K. Reddy, C. Mallard, J. Guan, K. Marks, L. Bennet, M. Gunning et al., Maturational change in the response to hypoperfusion injury in the fetal sheep. Pediatr Res 43 (1998), pp. 674-682

17. M.L. Reynolds, C.A.N. Evans, E.O.R. Reynolds, N.R. Sauders, G.M. Durbin and J.S. Wigglesworth, Intracranial haemorrhage in the preterm sheep fetus. Early Hum Dev 3 (1979), pp. 163-186.

18. S. Helou, R.C. Koehler, C.A. Gleason, M.D. Jones and R.J. Traystman, Cerebrovascular autoregulation during fetal development in sheep. Am J Physiol 266 (1994), pp. H1069-H1074

19. H.C. Lou, The "lost autoregulation hypothesis" and brain lesions in the newborn, an update. Brain Dev 10 (1988), pp. 143-146

20. F. Archbald, U.L. Verma, N.A. Tejani and S.M. Handwerker, Cerebral function monitor in the neonate. II: Birth asphyxia. Dev Med Child Neurol 26 (1984), pp. 162-168.

21. I. Bjerre, L. Hellstrom-Westas, I. Rosen and N.W. Svenningsen, Monitoring of cerebral function after severe asphyxia in infancy. Arch Dis Child 58 (1983), pp. 997-1002

22. J.P.H. Reulen, A.W. Gavilanes, D. van Mierlo, C. Blanco, F. Spaans and J.S. Vles, The Maastricht Cerebral Monitor (MCM) for the neonatal intensive care unit. J Med Eng Technol 23 (1999), pp. 29-37.

23. J.E.H. Bunt, A.W.D. Gavilanes, J.P.H. Reulen, C.E. Blanco and J.S.H. Vles, The influence of acute hypoxemia and hypovolemic hypotension of neuronal brain activity measured by the cerebral function monitor in newborn piglets. Neuropediatrics 27 (1996), pp. 260-264.

24. H.H. Szeto, T.D.H. Vo, G. Dwyer, M.E. Dogramajian, M.J. Cox and G.

Senger, The ontogeny of fetal lamb electrocortical activity: A power spectral analysis. Am J Obstet Gynecol 153 (1985), pp. 462-466. 
CHAPTER 5.

THE INFLUENCE OF ACUTE HYPOXEMIA AND HYPOVOLEMIC HYPOTENSION ON NEURONAL BRAIN ACTIVITY MEASURED BY THE CEREBRAL FUNCTION MONITOR IN NEWBORN PIGLETS

J.E.H.Bunt, A.W.D.Gavilanes, J.P.H.Reulen, C.E.Blanco and J.S.H. Vles

Neuropediatrics 1996; 27:260-264 


\begin{abstract}
Little is known about the cerebral electrical respons to short periods of hypoxemia, hypotension and their combination. These conditions occur frequently in critically ill newborn infants; their cerebral electrical activity can be registrated easily with the Cerebral Function Monitor (CFM). Therefore we recorded on-line cortical electrical activity during hypoxemia and hypotension in 11 newborn piglets aged 13-18 days. Hypoxemia was induced by reducing inspired oxygen fraction. Hypotension was induced by withdrawal of blood. The experimental groups were: Group I: arterial oxygen saturation $\left(\mathrm{SaO}_{2}\right) 45-85 \%$, group II: $\mathrm{SaO}_{2}<45 \%$, group III: mean arterial pressure (MAP) $50-75 \mathrm{mmHg}$, group IV: MAP $<50 \mathrm{mmHg}$, group V: $\mathrm{SaO}_{2}<85 \%$ and MAP 50-75 mmHg and group VI: $\mathrm{SaO}_{2}<85 \%$ and MAP $<50$ $\mathrm{mmHg}$.

CFM registrated normal cortical electrical activity during periods of moderate or severe hypoxemia (group I and II) and during isolated moderate hypotension (group III). The cortical activity decreased significantly due to severe hypotension alone (group IV) and combined hypotension and hypoxemia (group V and VI).

Hypotension has a more potent effect on cortical electrical activity than hypoxemia in the newborn piglet. Cerebral electrical activity does not change during severe hypoxemia and moderate hypotension possibly due to cerebral flow regulation. CFM recorded decreased cerebral electrical activity during severe hypotension and hypotension with hypoxemia. CFM could provide invaluable data in severe ill newborns.
\end{abstract}
Abbreviations
CFM, cerebral function monitor
$\mathrm{SaO}_{2}$, arterial oxygen saturation
MAP, mean arterial pressure
QAo, aorta blood flow
CBF, cerebral blood flow

\title{
Introduction
}

Continuous monitoring of cardiac and respiratory rate, arterial oxygen saturation and invasive arterial blood pressure are part of modern intensive care routine. However cortical brain activity, which is supposed to be the main determinant of long term morbidity, is not routinely monitored because continuous use of conventional multichannel electroencephalography is technically complicated in the intensive care unit and difficult to interpret.

In the last decade, a simplified form of continuous EEG recording know as Cerebral Function Monitor has been developed for the use in neonates $(19,25)$. The CFM gives a one channel registration of cerebral activity from two biparietal electrodes and it is presented as a band. CFM was first introduced by Maynard et al. (17) and has been used in the adult mainly during cardiac surgery, monitoring of anaesthesia, anticonvulsant therapy and during intensive care $(1,6,18)$. CFM patterns recorded in neonates are related to postconceptional age, behavioural 
states, seizures, hypoxic-ischaemic encephalopathy and hypoglycemia $(11,12,13,25,26)$. These studies considered CFM representative of brain function and these authors described the normal patterns from the different age groups. However, few neonatal animal experiments or clinical studies have focused on the study of the short-term impact of hypoxemia and hypotension on neuronal brain activity recorded continuously by CFM. Newborn infants requiring intensive care management often present periods of hypoxia and hypotension affecting regional blood flows and compromising oxygen delivery. These events increase the risk of damaging the developing human central nervous system, resulting in permanent sequelae. Brain electrical function ceases at a higher level of cerebral oxygen delivery than the level associated with hypoxic-ischemic brain injury (3). So the use of CFM as an on line monitor might offer the possibility to detect critical changes in cortical activity and help to establish a proper direction of therapy in severely ill newborns.

The aim of this work was to determine the effects of hypoxemia, hypotension and the combination of both on cortical electrical activity measured by CFM in the newborn piglet.

\section{Materials and methods}

Eleven newborn piglets aged between 13 to 18 days and weighing 3.2 to $5.0 \mathrm{~kg}$ were used in this study. Surgery was performed under general anaesthesia, induced by using a mask with halothane $2 \%$ and later continued with pentobarbital i.v. (4 mg/kg/hr) and pancuronium i.v. (0.05 mg/kg/hr) in dextrose $10 \%$ in water (6 $\mathrm{ml} / \mathrm{hr}$ ) administered through a peripheral vein in the ear, and later through a femoral vein. Tracheostomy was performed, and an endotracheal tube 5.0 (ID mm) was inserted. The piglets were mechanically ventilated with a $\mathrm{FiO} 2$ of 0.21 (using an $\mathrm{O}_{2} / \mathrm{N}_{2}$ mixture), frequency of $40 / \mathrm{min}$, inspiratory gas flow $10 \mathrm{~L} / \mathrm{min}$, PEEP of $5 \mathrm{~cm}$ $\mathrm{H}_{2} \mathrm{O}$ and peak inspiratory pressure adjusted to achieve a $\mathrm{PaCO}_{2}$ of $4-6 \mathrm{kPa}$. A $4 \mathrm{Fr}$ fiber optic catheter (Oximetrix System, Abbott Laboratories, North Chicago, IL, USA) was placed in the femoral artery and the tip was advanced into the abdominal aorta for continuous registration of arterial oxygen saturation and arterial blood pressure. A $5 \mathrm{Fr}$ catheter was placed in the femoral vein for continuous administration of fluids, anesthesia and for withdrawal of blood to induce hypotension.

A left intercostal $\left(5^{\text {th }}-6^{\text {th }}\right)$ thoracotomy was performed to place a $6 \mathrm{~mm}$ precalibrated ultrasonic transit time flow transducer around the aorta (Transonic Systems Inc., Ithaca NY 14850, USA), for continuous measurements of cardiac output. ECG electrodes were placed to monitor the heart rate continuously. Rectal temperature was maintained at $37^{\circ} \mathrm{C}$ using a heating mattress.

Three silver-plated isolated needle electrodes were inserted into the subcutis (two biparietal: P3 and P4; one frontal: F0, according to the 10 - 20 system of electrode placement, impedance: $<2 \mathrm{~K} \mathrm{Ohm}$ ) and connected to the CFM.

Arterial samples $(0.2 \mathrm{ml})$ were obtained every 10 minutes for measurement of blood gases and pH (AVL, radiometer, Copenhagen, Denmark) corrected for body temperature. Hemoglobin saturation was measured continuously with the fiber optic catheter, calibrated in vivo by using an hemoximeter (OSM2 hemoximeter, radiometer, Copenhagen, Denmark). Aortic blood flow (QAo), mean intra-arterial blood 
pressure (MAP) and heart rate values were sampled, digitalized and displayed every 10 seconds using a hemodynamic acquisition system (HDAS) developed at the University of Limburg. The study protocol was approved by the Medical Ethics Committee of the University of Limburg.

\section{Experiments}

The experiment consisted of four phases of hypoxemia and one phase of hypovolemic hypotension for each piglet. Two piglets were exposed to three phases of hypoxemia instead of four. Each experimental phase lasted 15 minutes and was followed by a recovery phase of 45 minutes. Mean arterial blood pressure during baseline recordings varied between 75 and $90 \mathrm{mmHg}$.

Systemic hypotension was defined as a MAP below $75 \mathrm{~mm} \mathrm{Hg}$ and was induced by a stepwise withdrawal of blood in total $30-50 \mathrm{ml} / \mathrm{kg}$ body weight from the venous or arterial catheter over a period of 30 minutes. Two levels of systemic hypotension were obtained: moderate systemic hypotension, MAP 50-75 mmHg (group III, $\mathrm{n=4}$ ) and severe systemic hypotension, MAP $<50 \mathrm{~mm} \mathrm{Hg}$ (group IV, $\mathrm{n=7}$ ). Recovery of hypotension was obtained by reinfusion of the volume of blood previously withdrawn.

Hypoxemia was defined as a $\mathrm{SaO}_{2}$ below $85 \%$ and it was induced by a stepwise reduction of $\mathrm{FiO}_{2}$ from 0.21 to 0.10 . The $\mathrm{SaO}_{2}$ obtained at $\mathrm{FiO} 20.10$ differed between experiments and animals and it defined the allocation to an experimental group. After 15 minutes $\mathrm{FiO}_{2}$ was increased to 0.21 (recovery). From a total of 42 hypoxemia experiments, 17 episodes corresponded to isolated hypoxemia. Two levels of isolated hypoxemia were obtained: $\mathrm{SaO}_{2} 45-85 \%$ (group I, $\mathrm{n}=12$ ) and $\mathrm{SaO}_{2}$ $<45 \%$ (group II, $n=5$ ). Furthermore, different levels of hypotension during hypoxemia were randomly present in 25 hypoxemia experiments: This combination was grouped as follows; $\mathrm{SaO}_{2}<85 \%$, MAP 50-75 mmHg (group V, $\mathrm{n}=16$ ) and $\mathrm{SaO}_{2}$ $<85 \%$, MAP $<50 \mathrm{mmHg}$ (group $\mathrm{VI}, \mathrm{n}=9$ ). Blood gases and $\mathrm{pH}$ abnormalities were corrected when necessary using sodium bicarbonate or adjusting mechanical ventilation. The experiment was ended by an overdose of pentobarbital.

\section{$\underline{\text { Calculations and data analysis }}$}

Sequential processing of the single channel EEG included: weighted digital band filtering between 2 and $15 \mathrm{~Hz}$, rectification, logarithmic amplitude compression and smoothing of the signal. For further analysis epochs of the CFM-signal lasting 3.6 seconds were selected. For each epoch a vertical line was drawn between the minimum and the maximum EEG - signal value. CFM recording was displayed on a computer screen and stored on digital audio tape. Epochs of 10 minutes of baseline CFM parameters (maximal, minimal and mean amplitude distribution and bandwidth in microvolts) were calculated by the computer using the software program Poly (Inspektor Research Systems BV., Amsterdam

The Netherlands) modified by the department of Clinical Neurophysiology, University of Limburg. The CFM and hemodynamic parameters (QAo, blood pressure and $\mathrm{HR}$ ) during experimental conditions were expressed as a percentage of baseline values. $\mathrm{PCO}_{2}$ and $\mathrm{pH}$ were expressed in absolute values. Mean and 
standard deviations of the percentages and absolute values in the different experimental groups were calculated, and statistically analyzed using the Wilcoxon matched pairs signed rank test. Statistical significance was considered at $p<0.05$.

\section{Results}

In the 11 newborn piglets 53 CFM epochs of 10 minutes were analyzed.

The tabel summarizes CFM, hemodynamic and blood gases parameters measured during the six different experimental situations: hypoxia (group I and II) , hypotension (group III and IV) and the combination of both (group V and VI).

During the two different levels of hypoxemia, group I: $\mathrm{SaO}_{2} 45$ to $85 \%(67 \pm$ $13.4 \%$, mean $\pm \mathrm{SD}, \mathrm{n}=12)$ and group II: $\mathrm{SaO}_{2}<45 \%(28.4 \pm 9.3 \%, \mathrm{n}=5)$, the obtained CFM and hemodynamic parameters were not different from baseline recordings (fig.1), except for a significant increase in heart rate in group II.

During moderate systemic hypotension in group III: MAP 50 to $75 \mathrm{~mm} \mathrm{Hg}(59.3 \pm$ $7.8 \mathrm{mmHg}, \mathrm{n}=4$ ) no difference in CFM and hemodynamic parameters could be observed compared to the baseline registration.

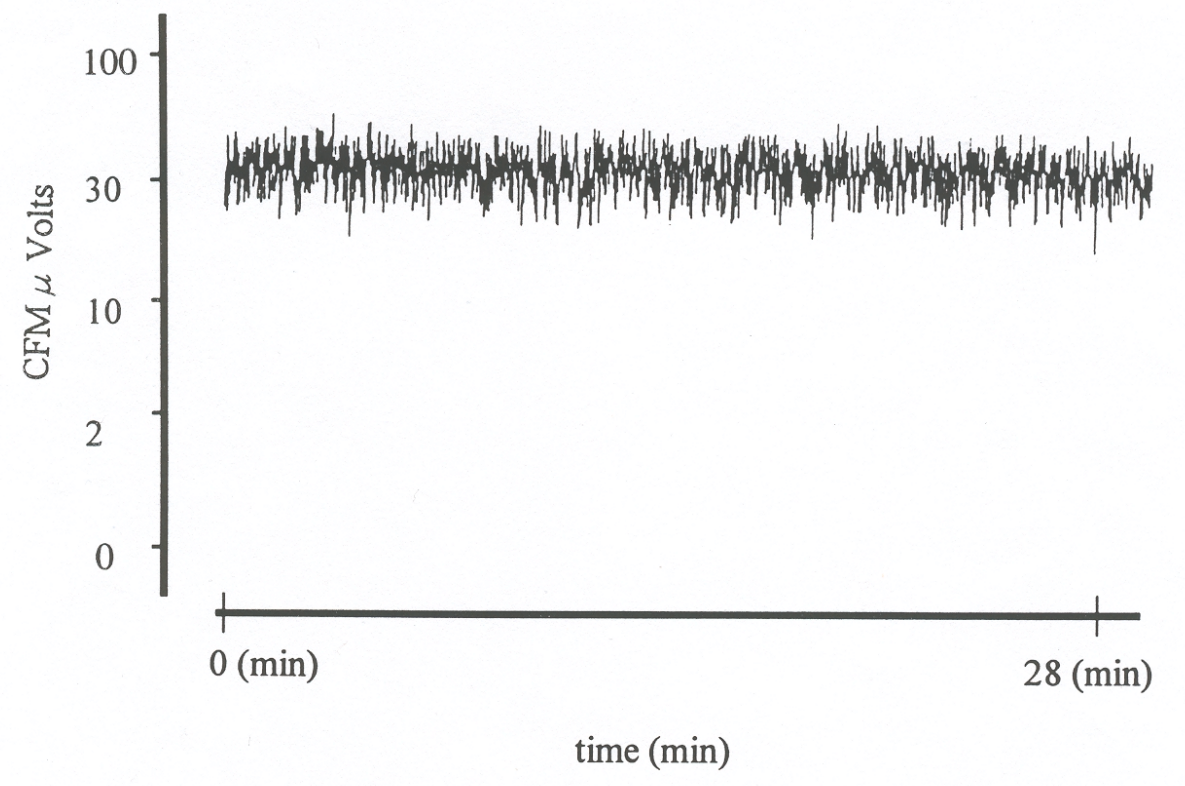

Fig.1 Baseline cortical activity during normoxemia and normotension recorded by CFM in a 15-days-old newborn piglet. 
However, during severe systemic hypotension (group IV: MAP $35.1 \pm 13.5 \mathrm{mmHg}, \mathrm{n}=7$ ), hypoxemia with moderate hypotension (group V: $\mathrm{SaO}_{2} 43.2 \pm 24.2 \%$ with MAP $62 \pm 10.3$ $\mathrm{mmHg}, \mathrm{n}=16$ ) and during hypoxemia with severe hypotension (group VI: $\mathrm{SaO}_{2} 31.4 \pm 15.4$ $\%$ with MAP $38.4 \pm 8.4 \mathrm{mmHg}, \mathrm{n}=9$ ), CFM parameters changed considerably compared to baseline values (fig.2). A significant decrease of all CFM parameters was observed in groups IV, V and VI (table). Aorta flow decreased during periods with severe hypotension, during moderate hypotension aorta flow did decrease but not significantly.

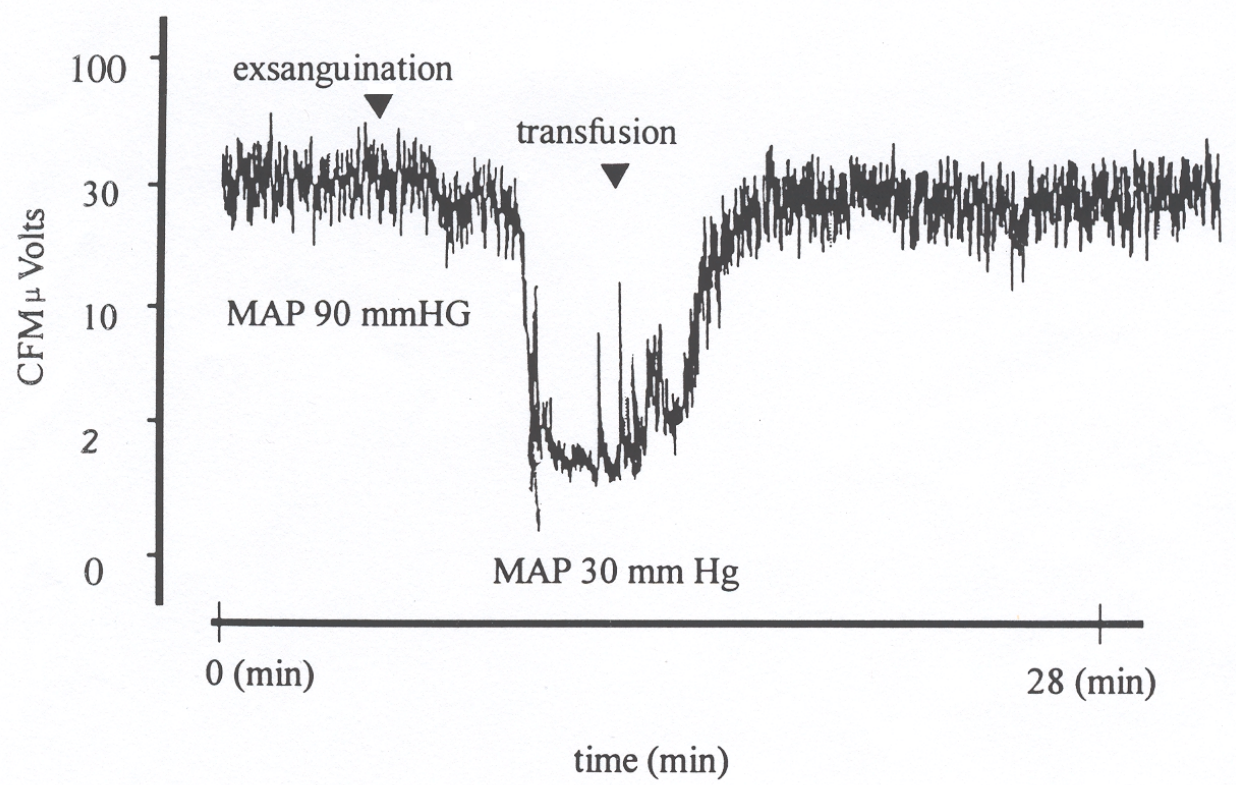

Fig.2.Cortical activity recorded with CFM during periods of provoked systemic hypotension and recovery. Note the immediate effect of hypotension and recovery in the amplitude of CFM recording. (MAP:mean arterial pressure). 


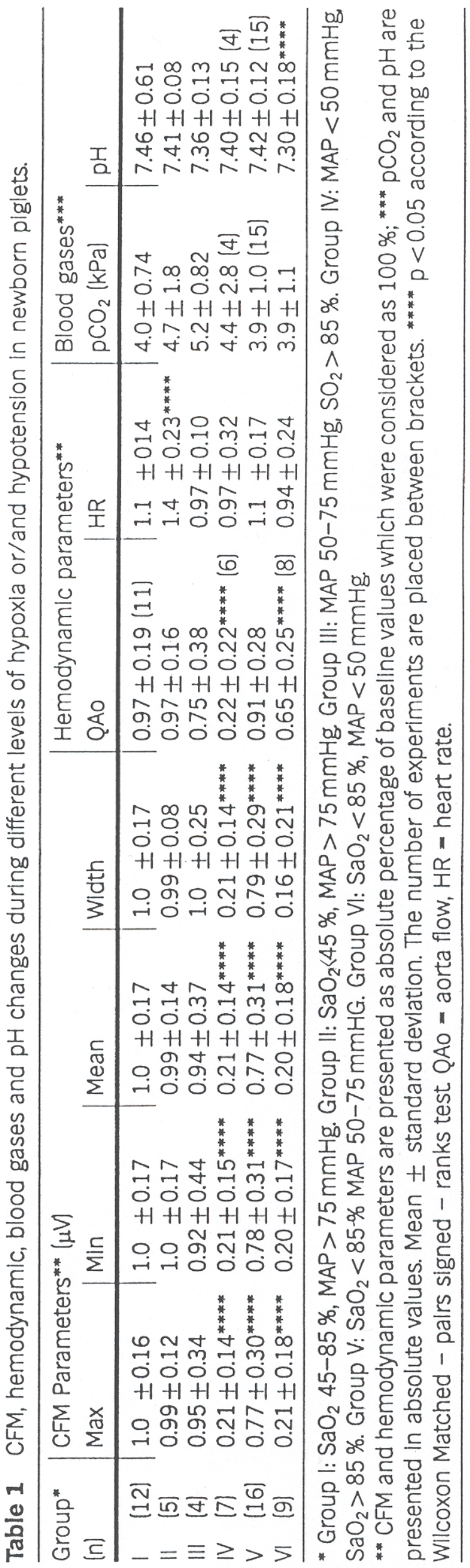




\section{Discussion}

The aim of this work was to study the influence of controlled hypoxemia and hypotension on cerebral cortical activity of the piglet measured by CFM. Our results showed that 15 minutes of isolated moderate or severe hypoxemia or moderate hypotension did not influence CFM patterns, whereas significant changes were obtained when severe isolated hypotension occured or when any degree of hypotension was combined with hypoxemia.

In our experiments, sodium pentobarbital was used as part of anesthesia and it is suggested in the literature that it could offer some neuroprotective effects, reducing $\mathrm{CBF}$ and cerebral metabolic rate (10). However, all animals received the same anesthetic, which makes all results comparable.

CFM is a simplified one channel EEG registration. The CFM trace gives the limits of the rectified amplitude distribution and is presented as a band. CFM provides a continuous picture of brain activity expressed in microvolts during different circumstances.

Compared with the conventional EEG, CFM allows long-term recording and on-line analysis with the additional advantage of simultaneous rejection of artifacts. CFM has already been introduced in neonatal intensive care units for the diagnosis of (sub)clinical seizures. It has been reported that CFM correlates well to the conventional multichannel EEG evaluation of cortical neuronal activity in neonates, except for the recognition of very short seizure patterns $(14,21)$. Moreover, the presence of recognizable CFM patterns could be useful as a long-term prognostic tool in case of severe peripartal asphyxia in full term infants $(2,4,16,27)$. However this method of continuous measurement of neuronal electrical activity has not yet been proven to be useful in acutely ill newborn infants. For this aim we planned experiments in piglets to reproduce common clinical situations in the neonatal period like hypoxemia, hypotension or the combination of both. Moreover, the brain of a one-week-old piglet is considered to be similar to this of the term human newborn infant with regard to the degree of maturation (20).

In our experiments, piglet cortical neuronal function registered with CFM remained unaffected despite periods of moderate or severe global hypoxemia. The only isolated factor, which affected cortical neuronal function, was severe hypovolemic arterial hypotension (group IV).

Combined hypotension and hypoxemia also affected cerebral function of the piglet (group V and $\mathrm{VI}$ ), in this experimental situation systemic hypotension did not have to decrease as much as in group IV to produce an effect on the CFM parameters.

These observations offer two important points for discussion: one is the lack of effect of hypoxemia regardless its severity, the other the relationship of neuronal activity and hypotension.

Alveolar hypoxemia is a very common procedure used in physiology

to challenge control systems, receptors and even cellular behavior. This procedure is based on the decrease in the oxygen tension in the alveoli and arterial blood, and the decrease in the hemoglobin saturation, all this leading to a decrease in oxygen content. Based upon this reasoning it could be proposed that the oxygen delivery to all body tissues would decrease equally and in direct proportion to the decrease in oxygen content. This is not correct since there are compensatory general 
mechanisms, which maintain and redistribute the blood flow to vital organs like heart and brain. This is the case of the brain, which receives an increase in blood flow to maintain an adequate oxygen delivery during hypoxemia (24). In summary, a decrease in $\mathrm{FiO}_{2}$ and in oxygen content does not necessarily produce changes at neuronal level, since as we previously discussed it can be compensated. The only way to know the real neuronal oxygenation status would be to measure oxygen tension intracellulaly. This will allow us to compare electrical activity with oxygenation status. The fact that we did not see changes in the CFM parameters could suggest that neuronal oxygenation was sufficient. Periods of isolated hypoxemia lasting 15 minutes seemed to be compensated through autoregulation.

In this study the episodes of hypoxemia lasted for 15 minutes. This is shorter than in other studies where decrease in electrical function and neurocellular changes were seen after longer periods of hypoxemia $(5,22)$. However, repetitive cycles of hypoxemia and reperfusion could cause a more severe energy failure. Furthermore, multiple ischemic insults in perinatal animal experiments have shown to produce striatal damage when compared to single insults which induce primarily cortical and hippocampal damage (9).

The second point of discussion is the association between systemic blood pressure and neuronal activity. Severe systemic hypotension or the combination of hypotension and hypoxemia caused very significant changes in all CFM parameters but were specially noticed in the baseline power of the electrical activity. These changes must have been produced by a decrease in CBF to critical levels which compromized oxygen delivery to the brain. The regulation of the cerebral perfusion is highly complex and ultimately involves cerebral vascular resistance, systemic blood pressure and cardiac output. CBF during hemorrhagic hypotension in newborn piglets is well maintained by cerebral vascular vasodilation with MAP as low as $35 \mathrm{mmHg}(7,28)$.

In our experiments the cardiac output was affected in two different ways. On the one hand we induced systemic hypotension through exsanguination of more than $30 \%$ of the circulating blood volume causing hypovolemic hypotension (groups III and IV). We believe that no anemic hypoxia took place as an additional factor, due to the acute character of the hypovolemia. On the other hand systemic hypotension was present in $60 \%$ of the global hypoxemia experiments, probably due to hypoxemic myocardial failure.

As shown in group IV and VI severe hypotension with and without hypoxemia caused a decrease in cardiac output (table), possibly affecting the cerebral perfusion and secondarily predisposing to cerebral corticoelectrical dysfunction measured by CFM. When moderate hypotension was combined with hypoxemia (group V) QAo remained unchanged compared to baseline, nevertheless this combined insult produced changes in neuronal activity most probably due to an impairment of CBF autoregulation. From our experiments we can hypothezise that arterial blood pressure is the most important single variable which can affect cerebral cortical function. This was partially suggested by Hellström-Westas et al. who reported a significant short decrease in cerebral cortical activity in preterm infants due to systemic hypotension after surfactant therapy (15).

The fact that neuronal activity recorded by CFM decreased only as a result of substantial hemodynamic changes and not during isolated hypoxemia suggests that the adaptative mechanisms to protect the brain offer a reasonable safety range. 
This is supported by observations where electrocortical silence is present only after a $60 \%$ reduction of CBF (8).

In conclusion, cortical electrical activity in piglets recorded with CFM

is decreased in the presence of severe hypotension or hypotension associated with hypoxemia. Moderate hypotension alone and isolated

hypoxemia of 15 minutes do not influence the cerebral cortical func-

tion of the piglet recorded by CFM. Systemic hypotension can affect neuronal activity, as measured by CFM, and its correction is essential in sick neonates who are at risk of hypoxemia due to a compromised respiratory status.

Continuous monitoring of cerebral cortical function using CFM is simple, noninvasive and is sensitive to acute and important changes in brain perfusion. This method could provide invaluable data when monitoring cerebral cortical activity in severely ill newborns.

\section{Acknowledgments}

The authors thank T. van der Nagel, Laboratory of Physiology, and D. van Mierlo, Laboratory of Clinical Neurophysiology, University of Limburg for their technical assistance. 


\section{References}

1. Altafullah, I., S.Asaikar, F.Torres: Clinical experience with two special devices for continuous cerebral monitoring. Acta Neurol. Scand. 84 (1991) 374-381

2. Archbald, F., U.L. Verma, N.A. Tejani, S.M. Handwerker: Cerebral function monitor in the neonate.II: Birth asphyxia. Dev. Med. Child. Neurol. 26 (1984) 162168

3. Astrup, J.: Energy-requiring cell functions in the ischemic brain. J. Neurosurg. 56 (1982) 482-497

4. Bjerre, I., L. Hellström-Westas, I. Rosén, N.W. Svenningsen: Monitoring of cerebral function after severe asphyxia in infancy. Arch. Dis. Child. 58 (1983) 9971002

5. DiGiacomo, J.E., : Effect of graded hypoxia on brain cell membrane injury in newborn piglets. Biol. Neonate 61(1992) 25-32

6. Dubois, M., T.M. Savege, T.M. O' Carrol, M. Frank: General anaesthesia and changes on the cerebral function monitor. Anaesthesia 33 (1978) 157-164

7 .Eidson, T.H., J.L. Edrington, M.L.C. Alburquerque, S.L. Zu

ckerman, C.WLeffler: Light/Dye microvascular injury eliminates pial arteriolar dilation in hypotensive piglets. Pediatr. Res. 37 (1994) 10-14

8. Ginsberg, M.D.: Neuroprotection in brain ischemia: An update (Part I). The Neuroscientist 1 (1995) 95-103

9. Gluckman, P.D., C.E. Williams: When and why do brain cells die? Developmental Medicine and Child Neurology 34 (1992) 1010-1021

10.Goodman Gilman, A., T.W. Rall, A. Nies, Peds Taylor P: Goodman and Gilman's The pharmacological basis of therapeutics, 8th. Ed. Pergamon Press Inc, New York, 1990, pp 302

11.Hellström-Westas, L., I. Rosén, N.W. Svenningsen: Silent seizures in sick infants in early life. Acta Paediatr. Scand.74

(1985) 741-748

12.Hellström-Westas, L., I. Rosén, N.W. Svenningsen: Cerebral complications detected by EEG-monitoring during neonatal intensive care. Acta Paediatr. Scand. suppl. 360 (1989) 83-86

13. Hellström-Westas, L., I. Rosén, N.W. Svenningsen: Cerebral function monitoring during the first week of life in extremely small low birthweight (ESLBW) infants. Neuropediatrics 22 (1991) 27-32

14. Hellström-Westas, L.: Comparison between tape-recorded and amplitude-integrated EEG monitoring in sick newborn infants. Acta Paediatr. Scand 81 (1992) 812-819

15.Hellström-Westas, L., A.H. Bell, L. Skov, G. Greisen, N.W. Svenningsen: Cerebroelectrical depression following surfactant treatment in preterm neonates. Pediatrics 89 (1992) 643-647

16.Hellström-Westas, L., I. Rosén, N.W. Svenningsen: Predictive value of early continuous amplitude integrated EEG recordings on outcome after severe birth asphyxia in full term infants. Arch. Dis. Child. 72 (1995) F34-F38

17.Maynard, D., P.F. Prior, D.F. Scott: Device for continuous monitoring of cerebral activity in resuscitated patients. Br. Med. J. 4 (1969) 545-546 
18.Prior, P.A., D.E. Maynard, P.C. Sheaff, B.R. Simpson, Strunin, E.J.M. Weaver, D.F. Scott: Monitoring cerebral function: Clinical experience with new device for continuous recording of electrical activity of brain. Br. Med. J. 2 (1971) 736-738 19.Prior, P., D. Maynard: Monitoring cerebral function. Long-term monitoring of EEG and evoked potentials. Amsterdam, Elsevier (1986)

20.Raju, T.N.K.: Some animals models for the study of perinatal asphyxia. Biol. Neonate 62 (1992) 202-214

21.Thiringer, K., J. Connel, R. Oozeer, E. Carter, M. Levene: Comparison between continuous Medilog EEG and Cerebral Function Monitor recordings on infants in neonatal intensive care. Early Hum. Develop. 14 (1986) 150-151

22. Thoresen, M., K. Haaland, E.M. Loberg, F. Apricena, E. Hanko, A. Whitelaw, et al.: A piglet model for posthypoxic encephalopathy. Pediatr. Res. 38 (1995) 457

23. Thornberg, E., K. Thiringer: Normal pattern of the cerebral function monitor trace in term and preterm neonates. Acta Paediatr. Scand.79 (1990) 20-25

24.Thorburn, K.L., M.J. Morton: Development of the Cardiovascular System. In: Thorburn GD, R (eds) Harding: Textbook of Fetal Physiology. Oxford, Oxford University Press, (1994) pp 126-128

25.Verma, U.L., F. Archbald, N.A. Tejani, S.M. Handwerker: Cerebral function monitor in the neonate. I: Normal patterns. Dev. Med. Child. Neurol. 26 (1984) 154161

26.Viniker, D.A., D.E. Maynard, D.F. Scott: Cerebral function monitor studies in neonates. Clin.Electroencephalogr. 15 (1984) 185-192

27.Wertheim, D., E. Mercuri, J.C. Faundez, M. Rutherford, D. Acolet, L. Dubowitz: Prognostic value of continuous electroencephalographic recording in full term infants with hypoxic ischaemic encephalopathy. Arch. Dis. Child. 71 (1994) F97F102

28. Yonetani, M., Ch Huang, J. McGowan, N. Lajevardi, A. Pastuszko, M. DelivoriaPapadopoulos, D.F. Wilson: Effect of hemorrhagic hypotension on extracellular level of dopamine, cortical oxygen pressure and blood flow in brain of newborn piglets. Neuroscience Letters 180 (1994) 247-252 
CHAPTER 6.

\section{ELECTROCORTICAL BRAIN ACTIVITY, CEREBRAL HAEMODYNAMICS AND OXYGENATION DURING PROGRESSIVE HYPOTENSION IN NEWBORN PIGLETS}

AWD Gavilanes, JH Vles, K von Siebenthal, JP Reulen, FH Nieman, R van Sprundel, and CE Blanco.

Clin Neurophysiology 2001; 112(1): 52-59 


\begin{abstract}
Objective: To investigate the relationships between systemic and cerebral haemodynamics and oxygenation, and electroencephalogram (EEG) amplitude and frequency analysis studied by the cerebral function analysing monitor (CFAM) during progressive hypovolemic hypotension.

Methods: Six piglets of one week of age, weighing 1.9 to $3.4 \mathrm{~kg}$ were mechanically ventilated under 1 to $1.5 \%$ halothane anaesthesia. After a one hour stabilization, blood was withdrawn in aliquots of $10 \mathrm{ml} / \mathrm{kg}$ over 15 minutes up to a total of 40 to $60 \mathrm{ml} / \mathrm{kg}$. Arterial oxygenation was maintained at normal levels. Thereafter, the total blood volume previously withdrawn, was reinfused. Changes in near infrared spectroscopy (NIRS) parameters [cerebral oxidized cytochrome aa3 (Cytaa3), cerebral blood volume (CBV) or total haemoglobin (tHb: oxy- + deoxyhaemoglobin)], carotid blood flow $\left(Q_{\text {car }}\right)$, maximal EEG amplitude and EEG frequency percentages were analysed continuously.

Results: The EEG amplitude remained stable until the mean arterial blood pressure (MAP), $\mathrm{Q}_{\mathrm{car}}$ and $\mathrm{tHb}$ dropped below $30 \mathrm{mmHg}(41 \%$ of baseline), 20 $\mathrm{ml} / \mathrm{min}$ (33\% of baseline) and $82 \%$ of baseline respectively. Delta $(\delta)$ wave frequency percentage of the CFAM increased significantly at MAP below $30 \mathrm{mmHg}$. EEG amplitude remained depressed after blood reinfusion and haemodynamic recovery. Cytaa3 changes were not statistically significant, reflecting sufficient neuronal oxygenation.
\end{abstract}

Conclusion: Our results show that electrocortical function is affected only by profound systemic hypotension. This occurred at a higher level of cerebral oxygen delivery than the level associated with neuronal hypoxia and secondary cell damage.

\title{
Key words
}

Cerebral Function Analysing Monitor - Near Infrared Spectroscopy - HypoxicIschemic Encephalopathy - Piglet - Newborn

\section{Abbreviations}

\begin{tabular}{|c|c|c|}
\hline CFM & & cerebral function monitor \\
\hline CFAM & & cerebral function analysing monitor \\
\hline CBF & & cerebral blood flow \\
\hline CBV & & cerebral blood volume \\
\hline Cytaa3 & & oxidized cytochrome aa3 \\
\hline deoxy-Hb & & deoxyhaemoglobin \\
\hline ECBA & : & electrocortical brain activity \\
\hline MAP & & mean arterial blood pressure \\
\hline NIRS & & near infrared spectroscopy \\
\hline $\mathrm{oxy}-\mathrm{Hb}$ & & oxyhaemoglobin \\
\hline$Q_{\text {car }}$ & & carotid blood flow \\
\hline QEEG & & quantitative electroencephalogram \\
\hline $\mathrm{tHb}$ & & oxy- + deoxyhaemoglobin \\
\hline
\end{tabular}




\section{Introduction}

The changes in neuronal function due to compromised oxygen delivery, medication, metabolic states or traumatic injury is accompanied by changes in electrocortical brain activity and cerebral haemodynamics (Volpe, 1995; Wauquier, 1993; Siebenthal and Casaer, 1992). The monitoring of these parameters is a useful tool to detect compromised neuronal function (Connell et al., 1988; Hellström-Westas et al., 1991; Tsuji M et al.,1995). However, the techniques developed for on-line monitoring used in the neonatal intensive care unit (NICU) are mainly directed to systemic haemodynamic and respiratory variables.

The original cerebral function monitor (CFM) is an asymmetrical filtered, amplitude integrated quantitative EEG (QEEG) (Maynard et al., 1969). Contrary to the monitoring of EEG, CFM does not require a skilled technician nor a considerable EEG expertise for its interpretation. CFM was primarily developed and used in adult intensive care, cardiac surgery and anaesthesia and it was later introduced in the care of neonates (Verma et al., 1984). CFM patterns have been described for different gestational ages, sleep-wake states, seizures, hypoglycaemia and to evaluate the effect of anticonvulsive treatment. Furthermore, CFM has been claimed to have a high prognostic value after birth asphyxia in full-term neonates (Eken et al., 1995; Hellström-Westas et al., 1995a,Thornberg and Ekström-Jodal, 1994). Additionally, CFAM analyses the EEG frequency content (Maynard, 1979). Available studies have been focussed on the description of clinical and experimental changes in EEG amplitude registered by CFM in adults and newborns (Prior et al., 1971; Thoresen et al.,1995; Thornberg and Thiringer, 1990; Bunt et al., 1996). Less attention has been given to the EEG frequency content.

NIRS is a noninvasive optical technique for assessing circulation and oxygenation (Siebenthal and Casaer, 1992). This method allows measurement of changes in cerebral oxyhaemoglobin (oxy-Hb), deoxyhaemoglobin (deoxy- $\mathrm{Hb}$ ), total $\mathrm{Hb}(\mathrm{tHb})$ and in the level of Cytaa3. Changes in concentration of $\mathrm{tHb}$ will reflect changes in CBV (Tsuji et al., 1995; Bucher et al., 1994). Cytaa3 is the terminal receptor for oxygen of the mitochondrial electron transport chain. Recent data support the use of NIRS to determine cerebral haemodynamics and cellular respiration (Tsuji et al., 1995).

The aim of this work was to study the effect of controlled changes in cerebral oxygen delivery on cortical neuronal function as monitored by CFAM. This information will help to understand the value of continuous monitoring of cerebral electrical activity in seriously compromised neonates.

\section{Materials and methods}

\section{Animal preparation}

The experiments were approved by the committee for animal experimentation of the University of Maastricht. Six newborn piglets of either sex, aged 7 days and weighing 1.9 to $3.4 \mathrm{~kg}$ were used in this study. Endotracheal intubation via tracheostomy with an endotracheal tube 4.0 (ID mm) was performed after halothane $2 \%$ induction with a mask. The piglets were mechanically ventilated using a Bourns BP 200 ventilator with a FiO2 of 0.21 , ventilatory rate of 40 breath $/ \mathrm{min}$, PEEP of 5 
$\mathrm{cm} \mathrm{H}_{2} \mathrm{O}$ and peak inspiratory pressure adjusted as necessary to provide a $\mathrm{P}_{2} \mathrm{O}_{2}$ of $10-15 \mathrm{kPa}, \mathrm{P}_{\mathrm{a}} \mathrm{CO}_{2}$ of 5-6 $\mathrm{kPa}$ and a $\mathrm{pH}$ between 7.35 and 7.45. Anaesthesia was further continued with halothane 1 to $1.5 \%$. ECG electrodes were placed to monitor ECG and heart rate. A $6 \mathrm{~mm}$ precalibrated ultrasonic transit time flow transducer (Transonic Systems Inc., Ithaca NY 14850, USA) was placed around the right common carotid artery, for continuous measurement of $Q_{\text {car }}$.

A 4 French fibre optic catheter (Oximetric System, Abbott Laboratories, North Chicago, IL, USA) was placed in the right femoral artery and the tip was advanced into the abdominal aorta for registration of arterial oxygen saturation $\left(\mathrm{SaO}_{2}\right)$ and systemic blood pressure. A 5 French catheter was placed in the femoral vein for the administration of dextrose $10 \%$ in water $(6 \mathrm{ml} / \mathrm{hr})$ to maintain blood glucose concentrations of $>4 \mathrm{mmol} / \mathrm{l}$. Another 5 French catheter was placed in the left femoral artery to take blood samples and for exsanguination. Rectal temperature was measured continuously and maintained between 38.0 and $39.0 \circ \mathrm{C}$ using a heating mattress and heating lamps. Silver-plated isolated needle electrodes were placed subcutaneously (two biparietal: P 3 and P 4; one frontal: F 0 , according to the $10-20$ system of electrode placement, impedance: $<2 \mathrm{~K} \mathrm{Ohm}$ ) and were connected to the CFAM. The NIRS sensor was placed in the midline (sagittal suture) fronto-parietally. The scalp was previously shaved and cleaned with alcohol to increase absorbency.

Arterial blood samples $(0.2 \mathrm{ml})$ were obtained every 15 minutes. Blood gases and $\mathrm{pH}$ (AVL, radiometer, Copenhagen, Denmark) were corrected for body temperature. $\mathrm{SaO}_{2}$ was measured continuously with the fibre optic catheter, calibrated in vivo by using a hemoximeter (OSM2 hemoximeter, radiometer, Copenhagen, Denmark). $\mathrm{SaO}_{2}, \mathrm{Q}_{\mathrm{car}}, \mathrm{MAP}$ and heart rate values were digitized and displayed every second using a haemodynamic acquisition system (HDAS) developed at the University of Maastricht.

\section{Experiments}

The experimental protocol is shown in fig. 1. Progressive hypovolemic hypotension was induced after a one-hour stabilization. Blood, $20 \mathrm{ml} / \mathrm{kg}$ body weight was first withdrawn during a 15-minute period and continued in aliquots of $10 \mathrm{ml} / \mathrm{kg}$ over 15 minutes. The withdrawn blood volume required to obtain a MAP below $30 \mathrm{~mm} \mathrm{Hg}$ and severe bradycardia was $40 \mathrm{ml} / \mathrm{kg}(\mathrm{n}=2), 50 \mathrm{ml} / \mathrm{kg}(\mathrm{n}=2)$ and $60 \mathrm{ml} / \mathrm{kg}(\mathrm{n}=2)$. This exsanguination phase is called a crash period.

Every exsanguination period was followed by a steady state of $15 \mathrm{~min}$. Blood gases and $\mathrm{pH}$ were corrected if needed during the steady states using sodium bicarbonate, tromethamine or changing ventilatory settings. Animals were under normoxemic conditions during the whole experiment. The crash period was followed by a steady state. Thereafter, the total blood volume previously withdrawn was reinfused over 10 to 15 - minute period. A recovery phase of 15 minutes followed the reinfusion of blood. At the end of the experiments all piglets were sacrificed with an overdose of pentobarbital. 
Data sampling and analysis

The CFAM - signal is an amplified, asymmetrical filtered, amplitude integrated three-channel EEG (P 3 - F 0, P 4 - F 0 and P 3 - P 4). EEG amplitude and frequency content were registered by CFAM using the software program Poly (Inspektor Research Systems BV., Amsterdam, The Netherlands). The CFAM system used in this study has been developed by the department of Clinical Neurophysiology, University of Maastricht (Reulen et al., 1999). CFAM depicted three-channel unprocessed EEG, EEG amplitude and EEG frequency analysis. Epochs of two seconds were analysed by the computer yielding EEG amplitude (bandwidth, minimum and maximum mean) and frequency percentages of delta [1$3.5 \mathrm{~Hz}]$, theta [3.5-7.5 Hz], alpha [7.5-13 Hz] and beta [13-27Hz]). Oxy-Hb and deoxy-Hb concentration and Cytaa3 were continuously registrated by a NIRSsystem (Critikon, Cerebral RedOx Monitor 2020, Johnson \& Johnson Medical). THb was calculated from the sum of oxy- $\mathrm{Hb}$ and deoxy- $\mathrm{Hb}$ and was used as an index of CBV. Changes in $\mathrm{tHb}$ are expressed as absolute percentage of baseline values, which were considered as $100 \%$. Cytaa3 results are changes from the baseline value and are expressed in $\mu \mathrm{mol} / \mathrm{L}$. The optodes of this NIRS reflectance system are placed in a one-piece probe and the transmitted light is detected by two detector channels at prefixed emitter-detector spacings of $10 \mathrm{~mm}$ and $35 \mathrm{~mm}$. The proximal detector collects light that has been predominantly transilluminated by extracerebral tissue. To the contrary, the distal detector is reached by light that has traveled deeper into the brain tissue. The NIRS-Critikon, Cerebral RedOx Monitor 2020 calculates Cytaa3 changes using the ratio of light detected in the two detectors. We assumed an optical pathlength of 4.4 times the interoptode distance (Delpy et al., 1988).

\section{Statistical Analysis}

Data acquired according to the experimental scheme showed in fig. 1 had to be trimmed down for each piglet individually. Steady states 3, 5, 7 and 9 were left out for the analysis and experimental phases were redefined into new periods. For each piglet the crash phase was divided into a period in which MAP $>30 \mathrm{mmHg}$ and one in which MAP $<30 \mathrm{mmHg}$ (Bunt et al., 1996) (fig. 2, periods $0>30 \mathrm{mmHg}$ and $0<30 \mathrm{mmHg}$ ). Using the crash moment between these new periods as a reference point only the last and foregoing last exsanguination phases preceding period $0<30 \mathrm{mmHg}$ were included in the analysis (fig.2, periods -2 and -1 ). The reason for this was that two piglets reached their crash period within the third exsanguination phase, two piglets within the fourth one and two piglets within the fifth exsanguination phase. The baseline measurements, the postcrash steady state, the blood reinfusion phase and the recovery phase were each maintained and redefined as new periods $-3,1,2$ and 3 respectively (fig. 2). The last ten minutes of each period were analysed. Univariate trend analysis and bivariate, correlational trend analysis have been done within a repeated measurements analysis of variance ANOVA. For the univariate trend analysis reversed Helmert contrasts have been calculated for periods -3 to $0<30 \mathrm{mmHg}$, in which each mean - except that of the baseline - is contrasted with the mean of the preceding means. Three separate simple contrasts have been calculated between period means 1, 2 and 3 versus the baseline mean. The two-paired samples comparison of variance test was applied to test differences between the variances of the $\delta$ wave $\%$ baseline 
and the 3 post crash phases. Next, a mixed model repeated measurements ANOVA was done to assess bivariate, correlational trend analysis using CFAM and NIRS parameters as dependents and the MAP as an independent variable. The same contrasts were used as in the univariate analysis. Changes of $\mathrm{pH}$, blood gases, glycemia and blood haemoglobin between baseline and the analysed experimental phases were compared by the Wilcoxon matched-pairs signed-rank test. Tests were considered to be statistically significant at $p<0.05$. Data are expressed as mean \pm SD. All calculations were made using SPSS programs, version 6.1 and 7.0 (SPSS Inc., Chicago, Illinois, USA).

\section{Results}

Table 1 summarizes the mean values for CFAM, NIRS and haemodynamic parameters during the experiment. Table 2 shows the arterial blood gases, glycemia and haemoglobin values measured during the experimental setting.

During progressive hypotension and before crash period:

Core temperature varied between 38.1 to $39.0^{\circ} \mathrm{C}$, blood glucose 3.1 to $6.4 \mathrm{mmol} / \mathrm{L}$, $\mathrm{P}_{\mathrm{a}} \mathrm{O}_{2} 11.5$ to $17.6 \mathrm{kPa}, \mathrm{P}_{\mathrm{a}} \mathrm{CO}_{2} 3.9$ to $5.9 \mathrm{kPa}, \mathrm{pH} 7.32$ to 7.42 and blood haemoglobin 2.2 to $6.6 \mathrm{~g} / 100 \mathrm{ml}$.

A positive significant correlation was found between $Q_{c a r}$, tHb and MAP. No difference in the EEG amplitude was observed compared to baseline registration. Unlike EEG amplitude, $\delta$ wave percentage began to increase at MAP below 47.9 $\mathrm{mmHg}(67 \%$ of baseline). The negative correlation was significant $(p=0.0069)$. Cytaa3 did not change significantly.

Within and after crash period:

Core temperature was 38.0 to $39.00^{\circ} \mathrm{C}$, blood glucose of 2.9 to $17 \mathrm{mmol} / \mathrm{L}, \mathrm{P}_{\mathrm{a}} \mathrm{O}_{2}$ of 10.1 to $21.4 \mathrm{kPa}, \mathrm{P}_{\mathrm{a}} \mathrm{CO}_{2}$ of 2.71 to $3.82 \mathrm{kPa}, \mathrm{pH}$ between 7.07 and 7.42 and haemoglobin of 1.9 to $4.8 \mathrm{~g} / 100 \mathrm{ml}$.

The maximal EEG amplitude $(p=0.002)$ decreased significantly when MAP and $Q_{\text {car }}$ dropped below $30 \mathrm{~mm} \mathrm{Hg}$ (41\% of baseline) and $20 \mathrm{ml} / \mathrm{min}$ (33\% of baseline) respectively. $\delta$ wave percentage increased significantly within the crash period $(p=0.0028)$. Thereafter, $\delta$ activity decreased significantly $(p=0.016)$. There was a trend toward a decrease in Cytaa3, but the difference was not statistically significant $(p=0.052)$.

During blood reinfusion and haemodynamic recovery:

Core temperature varied between 38.0 to $39.0^{\circ} \mathrm{C}$, blood glucose 2.4 to $7.1 \mathrm{mmol} / \mathrm{L}$, $\mathrm{P}_{\mathrm{a}} \mathrm{O}_{2}$ of 8.33 to $20.75 \mathrm{kPa}, \mathrm{P}_{\mathrm{a}} \mathrm{CO}_{2}$ of 3.55 to $7.67 \mathrm{kPa}, \mathrm{pH}$ between 6.78 and 7.50 and haemoglobin of 1.6 to $5.9 \mathrm{~g} / 100 \mathrm{ml}$.

All variables did recover to baseline values, except for EEG amplitude. EEG amplitude was completely suppressed in one piglet despite fully haemodynamic recovery. The mean $\delta$ wave $\%$ recuperated baseline values. To the contrary, baseline and recovery variances were significantly different $(p=0.024)$. 
Fig. 1 Experimental scheme of progressive hypovolemic hypotension in newborn piglets $(n=6)$.

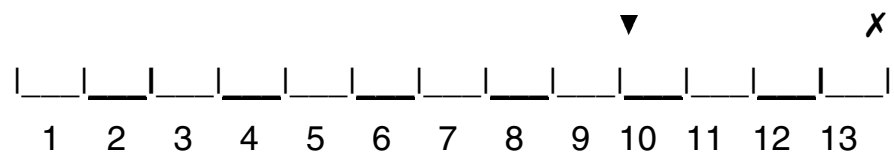

\section{NIRS and CFAM}

$\begin{array}{lll}1 & = & \text { baseline } \\ 2 & = & \text { exsanguination of } 20 \mathrm{ml} / \mathrm{kg} / 15 \mathrm{~min} \\ 3,5,7,9 & = & \text { steady states of } 15 \mathrm{~min} \\ 4,6,8 & = & \text { exsanguination of } 10 \mathrm{ml} / \mathrm{kg} / 15 \mathrm{~min} \\ 10 \nabla & = & \text { last exsanguination of } 10 \mathrm{ml} / \mathrm{kg} / 15 \mathrm{~min} \\ & & \text { or crash period } \\ 11 & = & \text { post-crash steady state } \\ 12 & = & \text { whole blood volume reinfusion } \\ 13 & = & \text { recovery phase } \\ x & = & \text { euthanasia }\end{array}$

Fig. 2 Experimental phases of progressive hypovolemic hypotension which were statistically analysed for this study.

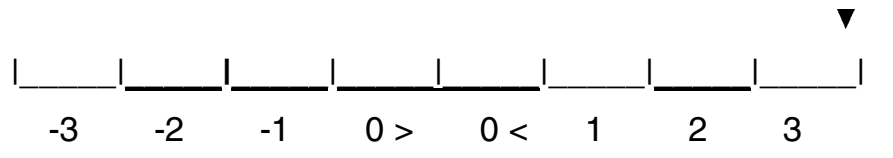

\section{NIRS and CFAM}

$\begin{array}{lll}-3 & = & \text { baseline } \\ -2,-1 & = & \text { first and second exsanguinations } \\ 0> & = & \text { last exsanguination with MAP }>30 \mathrm{mmHg} \\ 0< & = & \text { last exsanguination with MAP }<30 \mathrm{mmHg} \\ 1 & = & \text { post-crash steady state } \\ 2 & = & \text { whole blood volume reinfusion } \\ 3 & = & \text { recovery phase } \\ \nabla & = & \text { crash or severe hypotension and bradycardia }\end{array}$


Table 1

CFAM, NIRS and systemic haemodynamic parameters during progressive hypotension in newborn piglets.

\begin{tabular}{|c|c|c|c|c|c|c|}
\hline \multirow[b]{2}{*}{ phase } & \multicolumn{2}{|c|}{ Haemodynamic parameters } & \multicolumn{2}{|c|}{ CFAM parameters } & \multicolumn{2}{|c|}{ NIRS parameters } \\
\hline & $\begin{array}{c}\text { MAP } \\
(\mathrm{mmHg})\end{array}$ & $\begin{array}{l}\text { Qcar } \\
\text { (ml/min) }\end{array}$ & $\begin{array}{c}\text { ECBA } \\
\text { ( } \mu \text { volts) }\end{array}$ & $\begin{array}{c}\delta \text { wave } \\
(\%)\end{array}$ & $\begin{array}{c}\text { tHb } \\
(\% \text { from }-3)\end{array}$ & $\begin{array}{c}\text { Cytaa3 } \\
(\Delta \mu \mathrm{mol} / \mathrm{L})\end{array}$ \\
\hline-3 & $71.7 \pm 16.7$ & $43.6 \pm 17.5$ & $21 \pm 8$ & $38.8 \pm 4.9$ & $1.0 \pm 0.19$ & $0.0 \pm .4 .2$ \\
\hline-2 & $55.5 \pm 11.7^{*}$ & $29.2 \pm 6.99^{*}$ & $19.1 \pm 5.8$ & $41.8 \pm 2.7$ & $0.88 \pm 0.19^{*}$ & $0.18 \pm 3.9$ \\
\hline-1 & $47.9 \pm 10.5^{\star}$ & $23.4 \pm 4.89^{\star}$ & $18.4 \pm 5.4$ & $42.6 \pm 5.6$ & $0.87 \pm 0.18^{*}$ & $0.24 \pm 3.5$ \\
\hline $\begin{array}{c}0 \\
(\mathrm{MAP}>30 \mathrm{mmHg})\end{array}$ & $33.5 \pm 5.1^{*}$ & $17.3 \pm 3.8^{*}$ & $18.2 \pm 4.9$ & $45.3 \pm 7.8$ & $0.84 \pm 0.17^{*}$ & $0.20 \pm 3.2$ \\
\hline $\begin{array}{c}0 \\
(\mathrm{MAP}<30 \mathrm{mmHg})\end{array}$ & $18.2 \pm 7.8^{\star \star}$ & $9.7 \pm 3.7^{\star}$ & $9.9 \pm 6^{*}$ & $50.5 \pm 7.8^{\star}$ & $0.82 \pm 0.17^{*}$ & $-0.58 \pm 2.9$ \\
\hline 1 & $13.6 \pm 4.9^{\star \star}$ & $3.7 \pm 2.6^{\star}$ & $1.3 \pm 1.7^{\star \star}$ & $31.4 \pm 8.2^{*}$ & $0.76 \pm 0.15^{\star \star}$ & $-1.92 \pm 2.8$ \\
\hline 2 & $52.9 \pm 28.1$ & $25.7 \pm 18.8$ & $5.2 \pm 5.5^{\star *}$ & $38.6 \pm 9.4$ & $0.89 \pm 0.21$ & $-0.69 \pm 1.8$ \\
\hline 3 & $62.7 \pm 11.6$ & $54.1 \pm 15.3$ & $5.9 \pm 5.8^{*}$ & $39.4 \pm 10.41$ & $0.96 \pm 0.26$ & $-0.72 \pm 3.2$ \\
\hline
\end{tabular}

Qcar=carotid blood flow, ECBA =maximal mean electrocortical brain activity, tHb=total $\mathrm{Hb},-3=$ baseline, $-2,-1=$ first and second exsanguinations, 0 MAP $>30 \mathrm{mmHg}=$ last exsanguination with MAP $>30 \mathrm{mmHg}, 0$ MAP $<30 \mathrm{mmHg}=$ last exsanguination with MAP $<30 \mathrm{mmHg}, 1=$ post-crash steady state, $2=$ whole blood volume reinfusion, $3=$ recovery phase, ${ }^{*} p<0.05,{ }^{* *} p<0.005$ according to univariate ANOVA repeated measurements design, Helmert contrast among -3 to 0 MAP $<30 \mathrm{mmHg}$. I p 0.024 according to two paired samples comparison of variance among -3 to $0 \mathrm{MAP}<30 \mathrm{mmHg}$. Mean $\pm \mathrm{SD}$. 
Table 2 Changes of $\mathrm{pH}$, blood gases, glycemia and blood haemoglobin during progressive hypotension and blood reinfusion in newborn piglets.

\begin{tabular}{|c|c|c|c|c|c|}
\hline phase & $\mathrm{pH}$ & $\begin{array}{r}\mathrm{P}_{\mathrm{a}} \mathrm{O}_{2} \\
(\mathrm{kPa})\end{array}$ & $\begin{array}{r}\mathrm{P}_{\mathrm{a}} \mathrm{CO}_{2} \\
(\mathrm{kPa})\end{array}$ & $\begin{array}{l}\text { glycemia } \\
\text { (mmol/L) }\end{array}$ & $\begin{array}{c}\text { haemoglobin } \\
(\mathrm{g} / 100 \mathrm{ml})\end{array}$ \\
\hline baseline & $7.34 \pm 0.04$ & $13.13 \pm 1.7$ & $5.06 \pm 0.51$ & $4.82 \pm 1.22$ & $5.35 \pm 1.11$ \\
\hline $\begin{array}{l}\text { hypotension } \\
\text { (MAP>30mmHg) }\end{array}$ & $7.36 \pm 0.03$ & $14.69 \pm 1.8$ & $4.94 \pm 0.54$ & $4.54 \pm 1.83$ & $4.62 \pm 1.25$ * \\
\hline $\begin{array}{l}\text { haemodynamic crash } \\
(\mathrm{MAP}<30 \mathrm{mmHg})\end{array}$ & $7.29 \pm 0.16$ & $16.75 \pm 5.9$ & * $3.17 \pm 0.45$ & $9.93 \pm 7.05$ & $3.58 \pm 1.38$ * \\
\hline reinfusion-recovery & $7.16 \pm 0.23$ & $13.72 \pm 3.5$ & $5.15 \pm 1.52$ & $3.35 \pm 2.60$ & $4.31 \pm 1.70$ \\
\hline
\end{tabular}

${ }^{*} p<0.05$ according to Wilcoxon matched paired signed ranks test. Mean \pm SD.

\section{Discussion}

The purpose of this work was to study the effect of isolated progressive haemorrhagic hypotension on cerebral electrocortical activity, haemodynamics and oxygenation of newborn piglets measured by CFAM and NIRS respectively. Our results showed that a mean arterial pressure below $30 \mathrm{mmHg}$ affected cerebral perfusion and electrocortical activity as reflected in CFAM, when the oxygen supply was still sufficient to avoid neuronal hypoxia.

We chose the newborn piglet for our experiments because it is a well-documented model for the study of asphyctic insults (Raju et al., 1992; Goplerud et al., 1992; Yonetani et al., 1994; Tsuji et al., 1998). However, no information is available regarding experiments focussing on EEG spectra and amplitude analysis in combination with NIRS.

The brain tissue can be exposed before as well as after birth to many situations, which could compromise its function. The single most important factor which could induce loss of cerebral vascular autoregulation of the brain is systemic hypotension (Bunt et al., 1996; Hellström-Westas et al., 1992). Therefore, the study of the consequences of systemic hypotension on neuronal function could provide data of importance for the management of neonates at risk. Severe and prolonged hypoxia, hypotension or both lead to asphyxia. During asphyxia, compensatory mechanisms redistribute peripheral blood flow toward vital organs reducing peripheral oxygen consumption. In addition, cerebral vasodilatation assures appropriate cerebral oxygen delivery. However, when associated with severe systemic hypotension, cerebral perfusion is affected (Volpe, 1995; lacoboni et al., 1999). Because of loss 
of cerebral vascular autoregulation, cerebral blood flow (CBF) becomes pressure passive. Furthermore, energy failure leads to depletion of intracellular high-energy phosphate compounds, cytotoxic edema, primary and delayed secondary neuronal damage together with mitochondrial injury after reperfusion (Ginsberg, 1995; Gluckman and Williams, 1992). In piglets, the reduction in CBF is most pronounced in the cerebrum, less in the cerebellum, and least in the brainstem (Laptook et al., 1982). The consequences on later brain development depend on the severity of this regional damage.

In our model, we assured normocarbia, normoxia, normoglycemia and oxygen content. Thus, the only factor, which affected cerebral perfusion, was systemic hypotension. The level of glycemia increased during the haemodynamic crash period. This finding, although not significant, is indicative of a sympathoadrenal response to haemorrhagic stress. Finally, it is interesting to see that it was required up to $59 \%$ change of MAP and $67 \%$ of $Q_{c a r}$ to affect neuronal function. Qcar represented cerebral blood flow. $Q_{\text {car }}$ signal from the common carotid artery ultrasonic flow probe we used has been recently validated by Meadow et al.. $Q_{\text {car }}$ corresponded to cerebral blood flow determined by radioactive microspheres in a hypercarbic and hypoxic newborn piglet model (Meadow et al., 1999).

Conventional multichannel EEG and CFAM.

CFAM is a QEEG registration in which the EEG signal is filtered and selectively amplified. CFAM is easy to handle allowing continuous long-term bedside registration and on-line interpretation (Maynard and Jenkinson, 1984). Several studies showed that electrocortical brain activity (ECBA) depicted by CFAM correlates with that of a conventional EEG, except for the recognition of very short seizure patterns (Greisen, 1994; Hellström-Westas, 1992; Peck et al., 1981; Thiringer et al., 1986).

CFAM amplitude analysis

Since its first introduction by Maynard and Prior in 1969, an extensive body of information has been written concerning its use during adult cardiopulmonary surgery, anaesthesia and for determining brain death (Prior et al., 1971; Peck et al., 1981; Altafullah et al., 1991; Dubois et al.,1978; Patel, 1981). Normal patterns in premature and full-term infants have been described by several authors (HellströmWestas et al., 1991; Verma et al., 1984, Thornberg and Thiringer, 1990; Viniker et al., 1984). Changes in ECBA amplitude analysis registered by CFAM have been reported in several pathological conditions: hypoxic-ischaemic encephalopathy (Archbald et al., 1984, Bjerre et al., 1983; Wertheim et al., 1994), seizures (Hellström-Westas et al., 1985), monitoring anticonvulsant therapy (HellströmWestas et al., 1995b), after use of surfactant (Hellström-Westas et al., 1992a) and hypoglycaemia. In addition some authors proposed the use of CFAM amplitude patterns as a prognostic tool after hypoxic-ischaemic encephalopathy periods (Eken et al., 1995; Hellström-Westas et al., 1995a,Thornberg and Ekström-Jodal, 1994). Furthermore, the effects of hypoxia, ischaemia and anaesthetics on CFAM amplitude analysis have been studied using different animal models (Bunt et al., 1996; Aberg et al., 1989; Prior et al., 1978; Toner et al., 1995, Yuan et al., 1995). In our experiments, no burst-suppression activity was seen which means that halothane anaesthesia was appropriate. Hypovolemic hypotension was important 
and severe enough to induce significant systemic and cerebral haemodynamic changes. EEG symmetry during the experiments showed that ischaemia occurred in a global fashion. EEG amplitude decreased significantly compared to baseline values when MAP decreased below $30 \mathrm{mmHg}$. The impact on EEG amplitude remained even after blood reinfusion and in spite of systemic and cerebral haemodynamic recovery. This depressed EEG amplitude reflected the severity of the insult.

CFAM frequency analysis

Maynard reported in 1979 the first experience with EEG frequency analysis by CFAM. Since then, contrary to amplitude analysis, spectral analysis in physiological or pathological conditions has been not often reported. It has been reported in adults during surgery and anaesthesia (Sebel et al., 1983; Yate et al., 1986), during hemofiltration in patients with acute hepatic and renal failure (Davenport and Bramley, 1993), predicting neurological outcome after cardiopulmonary bypass surgery and in pediatric intensive care (Tasker et al., 1990). There are no data concerning clinical or experimental studies neither in human newborns nor in newborn animals.

Different EEG features have to be considered to evaluate spectral power. Such as dominance, reactivity, variability and topography of the EEG frequencies (Prior, $1993)$. In this study, $\delta$ frequency was analysed. The dominant frequency $\delta$ band was chosen for three reasons. First, the neonatal basic frequency spectra of EEG ranged between 3 to $4 \mathrm{~Hz}$, showing a dominant $\delta$ band. EEG spectral patterns similar to those found in adults develop later at 5 years of life. Secondly, inhalational anaesthetics slow the EEG spectral power (Wauquier, 1993). Additionally, halothane increases the dominant frequency prior to anaesthesia. These two factors explain the dominance of $\delta$ activity throughout the experiment. However, the experiments were started after one hour of stabilization under a constant dose of anaesthetic and further no changes in EEG amplitude or spectral power. Finally, increase in $\delta$ wave activity is associated with low levels of consciousness, severe hypocarbia, seizure disorders, cerebral trauma, post-hypoxic encephalopathy and brain ischaemia (Fisch, 1991).

In this work, although there were no significant differences, the increase in $\delta$ wave percentage activity was consistent when MAP was below $47 \mathrm{mmHg}(63 \%$ of baseline). The augmentation of $\delta$ wave activity within the crash period $(p=0.028)$ represented impairment of neuronal function. Mean $\delta$ wave \% recuperated baseline values during blood reinfusion and the recovery phase. However, the difference between baseline and recovery variances was statistically significant $(p=0.024)$. This phenomenon demonstrated the important heterogeneity of brain function during recovery.

Near infrared spectroscopy (NIRS)

NIRS provides a noninvasive tissue oxygenation and blood volume monitoring. The application of cerebral NIRS to animal studies was first reported by Jobsis in 1977 . Since then, several studies have validated and documented the value of NIRS in specific clinical and experimental situations (Jeni et al., 1996; Takei et al., 1993; Nomura et al., 1996). The NIRS-Critikon software calculates the changes in optical absorption of near infrared light and converts these into changes of $\mathrm{HbO} 2, \mathrm{Hb}, \mathrm{tHb}$ 
and the oxidation-reduction state of Cytaa3.

It was observed that MAP and Cytaa3 were independent from each other. Cytaa3 showed a consistent but not a significant increase during hypotension, as long MAP remained $>30 \mathrm{mmHg}$. In contrast, the haemodynamic crash and reperfusion were associated with an insignificant reduction of Cytaa3. Similar but significant Cytaa3 changes have been previously reported in other animal models and have been positive correlated with oxygen delivery. Tsuji et al. (1995) showed a significantly decreased Cytaa3 when MAP dropped below $17 \mathrm{mmHg}$ in combination with severe hypoxemia in the neonatal piglet. Shadid et al. reported a significant increase of Cytaa3 during hypercapnic hypoxia in newborn lambs, followed by an important decrease when haemorragic hypotension was added. In our experiments, in spite of severe systemic and cerebral haemodynamic changes at MAP $<30 \mathrm{mmHg}$, the tissue concentration of Cytaa3 decreased but showed no statistical significance. This suggests sufficient mitochondrial respiration during hypotension and reperfusion, reflecting the efficiency of adaptive mechanisms to maintain neuronal oxygen levels. The fact that Cytaa3 changes were not significant at any degree of hypotension might be due to the fact that normoxia was achieved throughout all the experimental phases. We did not performed jugular venous bulb oximetry, but we could assume that oxygen extraction was sufficient.

Critical values of MAP and $Q_{c a r}$ before the haemodynamic "crash" led to moderate but significant changes of $\mathrm{tHb}$ or CBV without an impact on ECBA. There is, however, an important fact to be pointed out regarding the interpretation of the $\mathrm{tHb}$ data. As shown in table 2, blood haemoglobin concentration decreased significantly during exsanguination. This haemorrhagic haemodilution invalidated the reliability of $\mathrm{tHb}$, since changes in concentration of $\mathrm{tHb}$ will reflect changes in CBV only if the blood Hb concentration remains stable (Tsuji et al., 1995; Bucher et al., 1994). Therefore, we speculate that changes of $\mathrm{tHb}$ or CBV were actually less important. This has been recently demonstrated in a piglet model of non-haemorrhagic hypotension (Tsuji et al., 1998).

The ranges for $\mathrm{P}_{2} \mathrm{CO}_{2}$ during hypotension and reperfusion were 2.71 to $7.67 \mathrm{kPa}$. Only the $\mathrm{P}_{\mathrm{a}} \mathrm{CO}_{2}$ variations during the crash period were significantly lower compared to baseline (table 2). Moderate hypocarbia leads to a reduction in CBF without changes in oxidative mechanisms. Only if $\mathrm{P}_{\mathrm{a}} \mathrm{CO}_{2}$ falls to less than $2.66 \mathrm{Kpa}$, the reduction is associated with mild reduction in cerebral oxidative mechanisms (Perlman, 1998). In our model, the pattern of CFAM changes during reperfusion and the unchanged redox state of Cytaa3 indicated the absence of permanent neuronal damage. Therefore, it can be assumed that hypocarbic vasoconstriction during the crash period was not enough to affect cerebral oxidative mechanisms (Wyatt et al., 1993).

In summary, our data show that neuronal activity is only affected when changes in cerebral perfusion are severe. Furthermore, electrocortical activity is depressed at a higher MAP and level of cerebral haemodynamics and oxygenation than those changes associated with neuronal hypoxia. Neuronal oxygenation might remain sufficient if normoxemia is assured during severe hypotension. These data support our primary statement that continuous monitoring of cerebral electrical activity is relevant during routine intensive neonatal care. 


\section{Acknowledgments}

The authors thank T. van der Nagel, Laboratory of Physiology, and D. van Mierlo, Department of Clinical Neurophysiology, University Hospital of Maastricht for their technical assistance. 


\section{References}

Aberg M TD, Rosen I D, Walther BD, Steen S. Cerebral Function Monitoring in Rats with a critical Hepatic Injury Treated with Pneumatic Antishock Garment and Infusion. J Trauma 1989;29(2):168-174.

Altafullah I, Asaikar S, Torres F. Clinical experience with two special devices for continuous cerebral monitoring. Acta Neurol Scand. 1991; 84:374-381.

Archbald F, Verma UL, Tejani NA, Handwerker SM. Cerebral function monitor in the neonate. II: Birth asphyxia. Dev Med Child Neurol 1984;26:162-168.

Bjerre I L, Hellström-Westas I, Rosén NW, Svenningsen. Monitoring of cerebral function after severe asphyxia in infancy. Arch Dis Child 1983;58:997-1002.

Bucher HU, Wolf M, Keel M, von Siebenthal K, Duc G. Effect of aminophylline on cerebral haemodynamics and oxidative metabolism in premature infants. Eur $\mathrm{J}$ Pediatr 1994;145:123-128.

Bunt JEH,Gavilanes AWD, Reulen JPH, Blanco CE, Vles JSH. The Influence of Acute Hypoxemia and Hypovolemic Hypotension of Neuronal Brain Activity Measured by the Cerebral Function Monitor in Newborn Piglets. Neuropediatrics 1996;27(5):260-264.

Connell J, de Vries L, Oozeer R, Rgev R, Dubowitz LM, Dubowitz V. Predictive value of early continuous electroencephalogram monitoring in ventilated preterm infants with IVH. Ped 1988;82(3):337-343.

Davenport A, Bramley PN. Cerebral Function Analysing Monitor and Visual Evoked Potential as a Non-invasive Method of Detecting Cerebral Dysfunction in Patients with Acute Hepatic and Renal Failure Treated with Intermittent Machine Hemofiltration. Renal Failure 1993;15(4):515-522.

Delpy DT, Cope M, van der Zee P,Arridge SR, Wray SC, Wyatt JS. Estimation of optical pathlength through tissue by direct time of flight measurement. PhysMed Biol 1988;33:1433-1442.

Dubois M, TM Savege, TM O' Carrol, M Frank. General anaesthesia and changes on the cerebral function monitor. Anaesthesia 1978;13:157-164.

Eken P, Toet MC, Groenendaal F, de Vries LS. Predictive value of early neuroimaging, pulsed Doppler and neurophysiology in full term infants with hypoxicischaemic encephalopathy. Arch Dis Child 1995;73: F75-F80.

Fisch BJ. Bilaterally synchronous slow waves. In: Spehlmanss=s EEG primer. Fisch BJ (ed) 2nd ed. Elsevier Science B.V., Amsterdam, The Netherlands, 1991;pp 441458. 
Ginsberg, MD. Neuroprotection in brain ischemia: An update (Part I). The Neuroscientist 1995;1:95-103.

Gluckman PD, Williams CE. When and why do brain cells die? Dev Med Child Neurol 1992;34:1010-1014.

Goplerud JM, Prakash Mishra O, Delivoria-padopoulos M. Brain Cell Membrane Dysfunction Following Acute Asphyxia in Newborn piglets. Biol Neonate 1992;61:33-41.

Greisen G. Tape-recorded EEG and the cerebral function monitor: amplitude-integrated, time-compressed EEG. J Perinat Med 1994;22:541-546.

Hellström-Westas L, Rosén I, Svenningsen NW. Silent seizures in sick infants in early life. Acta Paediatr Scand 1985;74:741-748.

Hellström-Westas L, Rosén I, Svenningsen NW. Cerebral function monitoring during the first week of life in extremely mall low birthweight (ESLBW) infants. Neuropediatrics 1991;22:27-32.

Hellström-Westas L, Bell AH, Skov L, Greisen G, Svenningsen NW.Cerebroelectrical depression following surfactant treatment in preterm neonates. Pediatrics 1992a;89:643-647.

Hellström-Westas L. Comparison between tape-recorded and amplitude-integrated EEG monitoring in sick newborn infants. Acta Paediatr Scand 1992b;81:812-819.

Hellström-Westas L, Rosén I, Svenningsen NW.Predictive value of early continuous amplitude integrated EEG recordings on outcome after severe birth asphyxia in full term infants. Arch Dis Child 1995a;72:F34-F38.

Hellström-Westas L, Blennow G, Lindroth M, Rosen I, Svenningsen NW. Low risk of seizure recurrence after early withdrawal of antiepileptic treatment in the neonatal period. Arch Dis Child 1995b;72:F97-F101.

lacoboni M, Baron JC, Frackowiak RS, Mazziotta JC, Lenzi GL. Emission tomography contribution to clinical neurology. Clin Neurophysiology 1999; 110: 2-23.

Jenni OG, Wolf M, Hengarter M, von Siebenthal K, Keel M, Bucher HU. Impact of Central, Obstructive and Mixed Apnea on Cerebral hemodynamics in Preterm Infants. Biol Neonate 1996;70:91-100.

Jobsis FF. Non-invasive infrared monitoring of cerebral and myocardial sufficiency and circulatory parameters. Science 1977;198:1264-1267. 
Laptook A, Stonestreet BS, Oh W. Autoregulation of brain blood flow in the newborn piglet: regional differences in flow reduction during hypotension. Early Hum Dev 1982; 6(1):99-107.

Maynard D, Prior PF, Scott DF. Device for continuous monitoring of cerebral activity in resuscitated patients. Br Med J 1969;4:545-546.

Maynard DE. Development of the CFM: The cerebral function analysing monitor (CFAM). Ann Anesth Franc 1979;3:253.

Maynard DE and Jenkinson JL. The cerebral function analysing monitor. Anesthesia Volume 1984;39:678-690.

Meadow W, Rudinsky B, Raju T, John E, Fornell L, Shankararao R. Correlation of flow probe determinations of common carotid artery blood flow and internal carotid artery blood flow with microsphere determinations of cerebral blood flow in piglets. Pediatr Res 1999; 45:324-330.

Nomura F, Naruse , du Plessis A, Hiramatsu T, Forbess J, Holtzman D, VolpeJJ, Jonas R, Tsuji M. Cerebral Oxygenation Measured by Near Infrared Spectroscopy during Cardiopulmonary Bypass and Deep Hypothermic Circulatory Arrest in Piglets. Pediatr Res 1996;40:790-796.

Patel $\mathrm{H}$. Experience with the cerebral function monitor during deliberate hypotension. Br F Anaesth 1981;53:639-645.

Peck Mc M RRT, Seriff NS, Wiener V, MD. Comparison of the cerebral function monitor with the EEG in determining brain death. Critical Care Medicine 1981;9 (6):459-463.

Perlman JM. Cerebral Blood Flow in Premature Infants: Regulation, urement, and Pathophysiology of Intraventricular Hemorrhage. In: Fetal and Neonatal Physiology. Polin RA and Fox WW (eds) 2nd ed. Saunders, 1998;pp 2175-2186.

Prior PA, Maynard DE, Sheaff PC, Simpson BR, Strunin L, Weaver EJ, Scott DF. Monitoring cerebral function: Clinical experience with new device for continuous recording of electrical activity of brain. Br Med J 1971;2:736-738.

Prior PF, Maynard DE, Brierley JB. EEG Monitoring for the control of anaesthesia produced by the infusion of althesin in primates. Br J Anaesth 1978;50:1993.

Prior P. Cerebral anoxia: Clinical aspects. In: Electroencephalography. Basic principles, clinical applications, and related fields. Niedermeyer E. and Lopes da Silva F (eds) 3th ed. Williams \& Wilkins, 1993;pp 431-44. 
Raju TNK. Some animals models for the study of perinatal phyxia.BiolNeonate 1992;62:202-214.

Reulen JPH, Gavilanes AWD , van Marl D, Blanco CE, Spaans F, Vles JSH. The Maastricht Cerebral Monitor (MCM) for the neonatal intensive care unit. Journal of Medical Engineering \& Technology 1999; 23(1):29-37.

Sebel PS, Maynard DE, Major E, Frank M. The cerebral function analysing monitor (CFAM). Br J Aneasth 1983;55:1265.

Shadid M, Hiltermann L, Monteiro L, Fontijn J, Van Bel F. Near infrared spectroscopy-measured changes in cerebral blood volume and cytochrome aa3 in newborn lambs exposed to hypoxia and hypercapnia, and ischemia: a comparison with changes in brain perfusion and O2 metabolism. Early Hum Dev 1999; 55(2): 169-82.

Siebenthal von K, Casaer GBP. Near-infrared spectroscopy in newborn infants. Brain Dev 1992; 14:135-143.

Takei J, Edwards AD, Lorek A, Peebles DM, Belai A, Cope M, Delpi Reynolds EOR. Effects of N-Nitro-L-Arginine Methyl Ester on the Cerebral Circulation of newborn Piglets Quantified In Vivo by Near-infrared Spectroscopy. Pediatr Res 1993; 34:354359.

Tasker RC, Boyd SG, Harden A, Matthew DJ. The cerebral function analysing monitor in paediatric intensive care: applications and limitations. Intensive Care Med 1990; 16:60-68.

Thiringer KJ, Connel R, Oozeer E, Carter M. Levene: Comparison between continuous Medilog EEG and Cerebral Function Monitor recordings on infants in neonatal intensive care. Early Hum Develop 1986; 14:150-151.

Thoresen M, Haaland K, Loberg EM, Whitelaw A, Apricena F, Hanko E, Steen PA. A piglet survival model for posthypoxic encephalopathy. Pediatr Res 1996; 40(5):738-748.

Thornberg E, Thiringer K. Normal pattern of the cerebral function monitor trace in term and preterm neonates. Acta Paediatr Scand 1990;79:20-25.

Thornberg $E$ and Ekström-Jodal B. Cerebral function monitoring: a method of predicting outcome in term neonates after severe perinatal asphyxia. Acta Paediatr 1994;83:596-601. 
Toner CC, Underwood MJ, Wright SJ, Cooper GJ, Reynols P, Withington PS. The cerebral function monitor (CFM) is a useful addition to a bilateral carotid artery, vein graft model. Laboratory Animals 1995;29:427-431.

Tsuji M, Naruse H, Volpe J, Holtzman D. Reduction of Cytochrome aa3 ured by Near-Infrared Spectroscopy Predicts Cerebral Energy Loss in Hypoxic Piglets. Pediatr Res 1995;37:253-259.

Tsuji M, Duplessis A, Taylor G, Crocker R, Volpe J. Near-Infrared Spectroscopy detects cerebral ischemia during hypotension in piglets. Pediatr Res 1998;44:591595.

Verma UL, Archbald F, Tejani NA, Handwerker SM. Cerebral function monitor in the neonate. I: Normal patterns. Dev Med Child Neurol 1984;26:154-161.

Viniker DA, Maynard DE, Scott DF. Cerebral function monitor studies in neonates. Clin Electroencephalogr 1984;15:185-192.

Volpe J. Hypoxic-ischemic encephalopathy: Biochemical and Physiological aspects. In: Neurology of the newborn. Volpe J. (ed) 3th ed. WB Saunders, Philadelphia, PA, 1995;pp 211-259.

Wauquier A. EEG and neuropharmacology. In Electroencephalography. Basic principles, clinical applications, and related fields. Niedermeyer E. and Lopes da Silva $\mathrm{F}$ (eds) 3th ed. Williams \& Wilkins, 1993;pp 619-629.

Wertheim D, Mercuri E, Faundez JC, Rutherford M, Acolet D, Dubowitz L. Prognostic value of continuous electroencephalographic recording in full term infants with hypoxic ischaemic encephalopathy. Arch Dis Child 1994;71:F97-F102.

Wyatt JS, Edwards AD, Reynolds EOR. Assesment of cerebral namics and oxygenation in the newborn infant. In: The Fetus and the Neonate; vol 1- The Circulation. Hanson M, Spencer JAD, Rodeck CH (eds) Cambridge University Press, Cambridge, 1993;pp 339-364.

Yate R, Maynard DE, Mayor E, Frank M, Verniquet AJW, Adams HK, Douglas EJ. Anaesthesia with ICI 35868 monitored by the cerebral function analysing monitor. European Journal of Anaesthesiology 1986;3:159-166.

Yonetani M, Huang Ch, McGowan J, Lajevardi N, Pastuszko A, Delivoria-padopoulos $\mathrm{M}$, Wilson DF. Effect of hemorrhagic hypotension on extracellular level of dopamine, cortical oxygen pressure and blood flow in brain of newborn piglets. Neuroscience Letters 1994;180:247-252. 
Yuan SZ, Runold M, Lagercrantz H. Cerebral and ventilatory depression during hypoxia in anaesthetized newborn guinea-pigs. Acta Paediatr 1995:84(12):14091413. 
CHAPTER 7.

\title{
NEONATAL ELECTROCORTICAL BRAIN ACTIVITY AND CEREBRAL TISSUE OXYGENATION DURING NON- ACIDOTIC, NORMOCARBIC AND NORMOTENSIVE GRADED HYPOXEMIA
}

\author{
AWD Gavilanes, JSH Vles, K von Siebenthal, R van Sprundel, JPH Reulen, and \\ CE Blanco.
}

Clinical Neurophysiology, in press 


\section{Abstract}

Objective: Neonates are commonly exposed to isolated hypoxemic episodes. In order to identify the risk of this, we correlate cerebral oxygen delivery and electrocortical brain activity during isolated graded and repetitive hypoxemia in 1week-old piglets.

Methods: Six halothane-anaesthetized piglets were subjected to two episodes of graded hypoxemia of 45 min-duration. The fractional concentration of inspired oxygen $\left(\mathrm{FiO}_{2}\right)$ was stepwise decreased at 15-min intervals from 0.21 to $0.15,0.10$ and 0.05. A second identical hypoxemic event was induced after one-hour of normoxemia $\left(\mathrm{FiO}_{2} 0.21\right)$. Mean arterial pressure (MAP) and $\mathrm{pH}$ were maintained at baseline values during the whole experiment. We measured near infrared spectroscopy parameters [cerebral oxidized cytochrome aa3 (Cytaa3), total haemoglobin (tHb: oxy- + deoxyhaemoglobin)] corresponding to cerebral blood volume $(\mathrm{CBV})$, carotid blood flow $\left(\mathrm{Q}_{\mathrm{car}}\right)$, intra-arterial oxygen saturation $\left(\mathrm{SaO}_{2}\right)$, and mean maximal EEG amplitude and relative spectral power.

Results: Delta $(\delta)$ power increased significantly and the EEG amplitude dropped below 10 and $5 \mu$ volts at the end of the first and the second hypoxemic period $\left[\mathrm{P}_{\mathrm{a}} \mathrm{O}_{2}\right.$ $2.68 \pm 1,08 \quad(p<0.05)$ and $2.87 \pm 0.58 \mathrm{kPa}$, respectively]. Both $E E G$ variables normalized during recovery $\left(\mathrm{FiO}_{2} 0.21\right)$. $\mathrm{Q}_{\mathrm{car}}$, CBV and Cytaa3 were not changed.

Conclusion: Acute isolated hypoxemia has to be sustained to induce neuronal hypofunction in normotensive animals. Hypoxic hypoxemia led to acute changes in neuronal activity, whereas cellular oxygenation remained unaffected.

\section{Key words}

Maastricht Cerebral Monitor - Electroencephalogram - Hypoxemia - Hypoxia - Near Infrared Spectroscopy - Newborn - Piglet

\section{Abbreviations}

$\begin{array}{lll}\text { CFM } & : & \text { cerebral function monitor } \\ \text { CFAM } & : & \text { cerebral function analising monitor } \\ \text { CBF } & : & \text { cerebral blood flow } \\ \text { CBV } & : & \text { cerebral blood volume } \\ \text { Cytaa3 } & : & \begin{array}{l}\text { oxidized cytochrome aa3 } \\ \text { deoxyhaemoglobin }\end{array} \\ \text { deoxy-Hb } & : & \text { electrocortical brain activity } \\ \text { EEG } & : & \text { electroencephalogram } \\ \text { MCM } & : & \text { Maastricht cerebral monitor } \\ \text { MAP } & : & \text { mean arterial blood pressure } \\ \text { NIRS } & : & \text { near infrared spectroscopy } \\ \text { NO } & : & \text { nitric oxide } \\ \text { oxy-Hb } & : & \text { oxyhaemoglobin } \\ Q_{\text {car }} & : & \text { carotid blood flow } \\ \mathrm{QEEG}_{\mathrm{SaO}} & : & \text { quantitative electroencephalogram } \\ \mathrm{THb} & : & \text { intra-arterial oxygen saturation } \\ & : & \text { total haemoglobin (oxy- + deoxyhaemoglobin) }\end{array}$




\section{Introduction}

Global ischemic and hypoxemic insults are common in neonatal intensive care and may cause permanent brain damage. Therefore, the on-line monitoring of cerebral oxygenation and neuronal function variables during neonatal intensive care could yield useful information complementary to systemic haemodynamic and respiratory variables.

During moderate hypoxemia, the sympathetic-adrenergic system is activated, redistributing blood flow in favor of brain, heart and adrenals. Concurrently, early electrical hypofunction is manifested (Volpe, 2001). The compensatory increased cerebral perfusion, and an accelerated compensatory anaerobic glycolysis, initially protects the brain tissue from hypoxemic metabolism. Severe hypoxemia, however, causes cardiovascular depression with significant systemic hypotension, impairing cerebral perfusion (Tsuji et al., 1995; Volpe, 2001).

Furthermore, loss of vascular autoregulation and a mitochondrial oxygen tension below $0.1 \mathrm{mmHg}$ provoke desaturation of the cytosolic energy system and compensatory glycolysis becomes inefficient. Therefore, EEG alterations seem to precede loss of autoregulation, metabolic depletion and cell damage. A potential explanation for these early electrical changes is the role of adenosine. Adenosine is increased in the interstitial space, releasing intracellular potassium and inhibiting postsynaptic potentials early during moderate hypoxemia. In addition, adenosine may further depress calcium conductance. The actions of adenosine on potassium and calcium metabolism make the cell less electrically excitable and spare cell energy avoiding metabolic failure and irreversible cell damage (Rothman, 1998).

The Maastricht Cerebral Monitor (MCM) and near infrared spectroscopy (NIRS) are two on-line tools to monitor cerebral electrocortical function and oxygenation changes, respectively. NIRS refers to an optical technique that assesses circulation and oxygenation. This method allows measurement of changes in cerebral oxyhaemoglobin (oxy- $\mathrm{Hb}$ ), deoxyhaemoglobin (deoxy- $\mathrm{Hb}$ ), total $\mathrm{Hb}(\mathrm{tHb})$ and in the level of Cytaa3. The MCM is a new computerized MCM system developed by the authors. It is an asymmetrical filtered, amplitude-integrated quantitative EEG (QEEG), which additionally analyses the EEG frequency content (Reulen et al., 1999). We first reported QEEG frequency spectral analysis in a piglet model of isolated hypovolemic hypotension (Gavilanes et al., 2001), using MCM and a NIRS apparatus. We showed that electrocortical function is affected only by profound systemic hypotension. This occurred at a higher level of cerebral oxygen delivery than the level associated with neuronal hypoxemia. In the present study the effect of repeated non-acidotic, normotensive hypoxic hypoxemia on cortical neuronal function and cerebral oxygen delivery is evaluated. MCM and NIRS are used as monitors to obtain more insights into the adaptive mechanisms of the neonatal brain subjected to impaired oxygenation. 


\section{Materials and methods}

\subsection{Animal and surgical procedures}

A total of six piglets of one week of age were used in accordance with the committee for animal experimentation of the University of Maastricht. Animals were initially anaesthetized using halothane $2 \%$ induction with a face mask and endotracheal intubated via tracheostomy with an endotracheal tube 4.0 (ID mm). The piglets were positive pressure ventilated (Bourns BP 200) with a fractional concentration of inspired oxygen $\left(\mathrm{FiO}_{2}\right)$ of 0.21 , a ventilation rate of 40 breaths/min, PEEP of $5 \mathrm{~cm} \mathrm{H}_{2} \mathrm{O}$ and PIP adjusted as necessary to provide acceptable chest wall movement and arterial blood gases $\left(\mathrm{P}_{\mathrm{a}} \mathrm{O}_{2}\right.$ of $10-15 \mathrm{kPa}, \mathrm{P}_{\mathrm{a}} \mathrm{CO}_{2}$ of $4.5-6 \mathrm{kPa}$ and $\mathrm{pH}$ of $\left.7.35-7.45\right)$. Anaesthesia was then maintained with halothane 1.0 to $1.5 \%$. Continuous three-lead ECG was recorded throughout the experiment. A $6 \mathrm{~mm}$ precalibrated ultrasonic blood volume flow probe (Transonic Systems Inc., Ithaca NY 14850, USA) was placed around the right common carotid artery to measure $Q_{c a r}$. The right femoral artery was cannulated with a 4 French fibre optic catheter (Oximetric System, Abbott Laboratories, North Chicago, IL, USA) to record systemic blood pressure and intra-arterial oxygen saturation $\left(\mathrm{SaO}_{2}\right)$. A 5 French catheter was placed in the left femoral artery to take blood samples. Another 5 French catheter was inserted into the femoral vein for the administration of D10W, $2.5 \mathrm{ml} / \mathrm{kg}$ per hour. Rectal temperature was monitored throughout the experiment and maintained between 38.0 and $39.0^{\circ} \mathrm{C}$ 's by the use of a heating mattress and heating lamps. Brain activity using MCM was monitored between three silver-plated isolated needle electrodes that were placed into the scalp (one midfrontal and two parietal electrodes, impedance: $<2 \mathrm{~K} \mathrm{Ohm}$ ). The NIRS sensor was placed in the midline fronto-parietally and fixed with bandages. The scalp was previously shaved and cleaned with alcohol to increase light penetration. Arterial blood samples $(0.2 \mathrm{ml})$ were obtained at 15 min-interval to determine serum glucose concentration, haemoglobin, blood gases and $\mathrm{pH}$ (ABL, Radiometer, Copenhagen, Denmark; measurements corrected for body temperature). $\mathrm{SaO}_{2}$ was measured continuously with the fibre optic catheter, and calibrated in vivo by using a hemoximeter (OSM2 hemoximeter, radiometer, Copenhagen, Denmark).

\subsection{Experimental protocol (fig.1)}

After one-hour initial period of postsurgical baseline measurements, the animals were challenged with two episodes of hypoxemia of 45 min-duration (episodes a and b). Each episode consisted of three phase of hypoxemia. Each phase lasted $15 \mathrm{~min}$. Hypoxemia was induced by changing the proportion of oxygen/nitrogen in the inspiratory gas. $\mathrm{FiO}_{2}$ was reduced from 0.21 to 0.15 , to 0.10 and finally to 0.05 . The second identical hypoxemic episode was induced after one-hour recovery/baseline under normoxic conditions $\left(\mathrm{FiO}_{2}\right.$ 0.21). $\mathrm{P}_{\mathrm{a}} \mathrm{CO}_{2}$ and $\mathrm{pH}$ were corrected if needed using sodium bicarbonate, tromethamine or changing ventilatory settings. Polygeline (Haemaccel, HMR) $10 \mathrm{ml} / \mathrm{kg}$ IV was used as volume expander when necessary to maintain MAP above $40 \mathrm{mmHg}$. At the end of the experiments all piglets were killed using a high-dose pentobarbital. 
Fig. 1

Experimental scheme of two episodes of graded hypoxic hypoxemia in newborn piglets $(n=6)$.

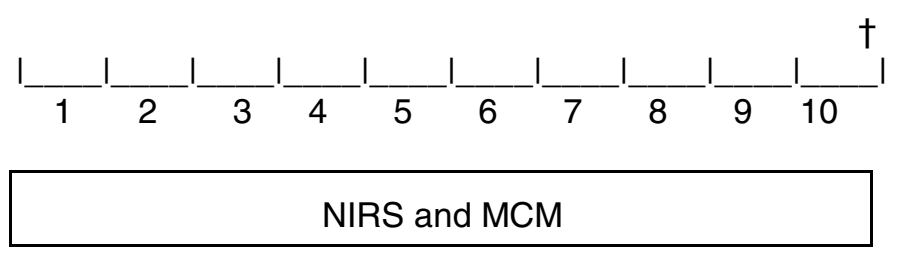

$\begin{array}{ll}\text { 1: } & \text { baseline a / } 1 \text { hour } \\ \text { 2: } & \mathrm{FiO}_{2} 0.15 / 15 \mathrm{~min} \\ \text { 3: } & \mathrm{FiO}_{2} 0.10 / 15 \text { min } \\ \text { 4: } & \mathrm{FiO}_{2} 0.05 / 15 \mathrm{~min} \\ \text { 5: } & \text { recovery a } \mathrm{FiO}_{2} 0.21 / 15 \mathrm{~min} \\ \text { 6: } & \text { baseline } \mathrm{b} / 45 \mathrm{~min} \\ \text { 7: } & \mathrm{FiO}_{2} 0.15 / 15 \mathrm{~min} \\ \text { 8: } & \mathrm{FiO}_{2} 0.10 / 15 \mathrm{~min} \\ \text { 9: } & \mathrm{FiO}_{2} 0.05 / 15 \mathrm{~min} \\ \text { 10: } & \text { recovery b } \mathrm{FiO} 0.21 / 15 \mathrm{~min} \\ \dagger: & \text { euthanasia }\end{array}$

\subsection{Analysis of data}

$\mathrm{SaO}_{2}, \mathrm{Q}_{\mathrm{car}}$, MAP and heart rate were monitored every second using a home-made haemodynamic acquisition system (HDAS).

The computerized MCM system used in the experiments has been developed by the authors, and it has been described before (Reulen et al., 1999). MCM depicted three-channel unprocessed EEG, EEG amplitude and EEG frequency analysis. Epochs of two seconds were analyzed by the computer yielding EEG amplitude (bandwidth, minimum, maximum and mean) and relative delta [1-3.5 Hz], theta [3.5$7.5 \mathrm{~Hz}]$, alpha [7.5-13 Hz] and beta [13-27Hz] power. Only the biparietal MCM recording was further analyzed and defined as ECBA.

The redox state of the $\mathrm{Hb}$ and cerebral Cytaa3 was calculated by a NIRSinstrument (Critikon, Cerebral RedOx Monitor 2020, Johnson \& Johnson Medical Ltd., Norderstedt, Germany) and saved every second. THb was calculated from the sum of oxy-Hb and deoxy- $\mathrm{Hb}$ and was used as an index of CBV. THb and Cytaa3 data are absolute changes from the baseline value and are expressed in $\mu \mathrm{mol} / \mathrm{L}$. The optodes of this NIRS reflectance system are placed in a one-piece probe and two detector channels at emitter-detector spacing of $10 \mathrm{~mm}$ and $35 \mathrm{~mm}$ detect the transmitted light. The proximal and the distal detectors perceive light that has been transilluminated by extracerebral tissue and brain tissue, respectively. Cerebral Cytaa3 is calculated by correction for the proximal sample. The optical pathlength of 4.4 times the interoptode distance (Delpy et al., 1988).

\subsection{Statistics}

The last five minutes of each experimental phase were analyzed. The second hypoxemic period was analyzed in four out of six animals (for details see results). 
Statistical analysis within each hypoxemic period and between the two hypoxemic periods was done by the non-parametric Wilcoxon matched-pairs signed-rank test. All calculations were made using SPSS programs, version 8.0 (SPSS Inc., Chicago, Illinois, USA). Data are expressed as mean \pm SD. The criterion for significance was $p<0.05$ level and highly significant at the $p<0.005$ level.

\section{Results}

Six male piglets, aged 7 days and weighing 2.3 to $3.9 \mathrm{~kg}$ were used in this study. Values for MCM, NIRS and haemodynamic parameters, and for arterial blood gases are shown in table 1 and 2. Core temperature $\left(38.0\right.$ to $\left.39.0^{\circ} \mathrm{C}\right)$, glycemia and haemoglobin remained within baseline ranges (data not shown). No significant differences in MCM, NIRS and haemodynamic parameters were found between the baseline phases of the two hypoxemic episodes.

\subsection{The first episode of graded hypoxemia, episode a $(n=6)$ :}

Compared to baseline, EEG amplitude and $\delta$ wave percentage remained unchanged during the first 30 minutes of hypoxemia $\left(\mathrm{FiO}_{2} 0.15-0.10\right)$. In the last 15 minutes $\left(\mathrm{FiO}_{2} 0.05\right.$ ), $\mathrm{SaO}_{2}$ below $24.1 \%$ (25\% of baseline) and $\mathrm{P}_{\mathrm{a}} \mathrm{O}_{2}$ of $2.68 \pm 1,08$ $\mathrm{kPa}$ induced significant changes in these two EEG variables $(p<0.05)$. All animals had unaltered $\mathrm{pH}$ and $\mathrm{P}_{\mathrm{a}} \mathrm{CO}_{2}$ above $3.95 \mathrm{kPa}$ during the whole hypoxic episode. MAP, $Q_{c a r}$, tHb and Cytaa3 did not change significantly.

\subsection{Recovery a: first reoxygenation and haemodynamic recovery $(n=6)$ :}

One piglet needed $10 \mathrm{ml} / \mathrm{kg}$ non-blood volume expanders to return MAP to values $>40 \mathrm{mmHg}$. Systemic oxygenation returned to baseline values within 5 minutes. The completely recovery of EEG amplitude and EEG $\delta$ frequency percentage took place at the end of this period.

\subsection{The second episode of graded hypoxemia, episode $b(n=4)$ :}

All animals survived the first hypoxemic episode, but one died after 30 min of normoxemia. Furthermore, data of one out of five survivors could not be further analyzed because of artefactual MCM data.

The maximal EEG amplitude decreased and the percentage $\delta$ power increased significantly $(p<0.05)$ when $\mathrm{SaO}_{2}$ dropped below $23.5 \%(25 \%$ of baseline, $\mathrm{p}<0.05)$. MAP, $\mathrm{pH}, \mathrm{Q}_{\mathrm{car}} \mathrm{t} \mathrm{Hb}$ and Cytaa3 remained unaltered.

\subsection{Recovery $b$ : the second reoxygenation and haemodynamic recovery $(n=2)$ :}

MCM and NIRS data of two animals were lacking. Therefore, this phase was not statistically analyzed. The few data suggested that the decrease of the EEG amplitude and increase in $\delta$ power were reversible. 
Table 2. Blood gas values during graded and repetitive hypoxemia in newborn piglets

\begin{tabular}{|c|l|l|l|}
\hline phase & \multicolumn{1}{|c|}{$\mathrm{pH}$} & \multicolumn{1}{c|}{$\begin{array}{c}\mathrm{P}_{\mathrm{a}} \mathrm{O}_{2} \\
(\mathrm{kPa})\end{array}$} & \multicolumn{1}{c|}{$\begin{array}{c}\mathrm{P}_{\mathrm{a}} \mathrm{CO}_{2} \\
(\mathrm{kPa})\end{array}$} \\
\hline $\begin{array}{c}\text { baseline a } \\
(\mathrm{n}=6)\end{array}$ & $7.36 \pm 0.03$ & $10.61 \pm 0.63$ & $5.20 \pm 0.43$ \\
\hline $\mathrm{FiO}_{2} 0.15 \mathrm{a}$ & $7.37 \pm 0.04$ & $6.27 \pm 0.98^{* *}$ & $4.75 \pm 0.42^{*}$ \\
\hline $\mathrm{FiO}_{2} 0.10 \mathrm{a}$ & $7.38 \pm 0.05$ & $2.96 \pm 0.40^{\star *}$ & $4.43 \pm 0.68$ \\
\hline $\mathrm{FiO}_{2} 0.05 \mathrm{a}$ & $7.34 \pm 0.05$ & $2.68 \pm 1.08^{*}$ & $3.95 \pm 1.33$ \\
\hline $\mathrm{recovery} \mathrm{a}^{*}$ & $7.33 \pm 0.06$ & $9.77 \pm 2.83$ & $4.09 \pm 0.19^{*}$ \\
\hline $\mathrm{baseline} \mathrm{b}_{(\mathrm{n}=4)}$ & $7.41 \pm 0.06$ & $9.91 \pm 0.90$ & $4.91 \pm 0.83$ \\
\hline $\mathrm{FiO}_{2} 0.15 \mathrm{~b}$ & $7.40 \pm 0.05$ & $8.33 \pm 1.67$ & $5.20 \pm 0.81$ \\
\hline $\mathrm{FiO}_{2} 0.10 \mathrm{~b}$ & $7.42 \pm 0.04$ & $4.95 \pm 1.64^{*}$ & $4.60 \pm 0.72^{*}$ \\
\hline $\mathrm{FiO}_{2} 0.05 \mathrm{~b}$ & $7.34 \pm 0.08$ & $2.87 \pm 0.58(\mathrm{n}=1)$ & $4.20 \pm 0.93$ \\
\hline $\mathrm{recovery} \mathrm{b}^{*}$ & $7.49 \pm 0.17(\mathrm{n}=2)$ & $10.70(\mathrm{n}=1)$ & $2.47 \pm 1.36(\mathrm{n}=2)$ \\
\hline
\end{tabular}

${ }^{*} p<0.05$ and ${ }^{* *} p<0.005$ according to non-parametric Wilcoxon matched-pairs signed-rank test.; $n=$ number of experiments. Data were not analyzed. Mean $\pm S D$. 
Table 1. MCM, NIRS, MAP,Qcar and haematologic values during graded hypoxemia

\begin{tabular}{|c|c|c|}
\hline HAEMODYNAMICS & NIRS & MCM \\
\hline
\end{tabular}

\begin{tabular}{|c|c|c|c|c|c|c|c|}
\hline phase & $\begin{array}{c}\mathrm{SaO} 2 \\
(\%)\end{array}$ & $\begin{array}{c}\text { MAP } \\
(\mathrm{mmHg})\end{array}$ & $\begin{array}{l}\text { Qcar } \\
(\mathrm{ml} / \mathrm{min})\end{array}$ & $\begin{array}{c}\mathrm{tHb} \\
(\mu \mathrm{mol} / \mathrm{L})\end{array}$ & $\begin{array}{l}\text { Cytaa3 } \\
(\mu \mathrm{mol} / \mathrm{L})\end{array}$ & $\begin{array}{c}\text { ECBA } \\
\text { ( } \mu \text { volts) }\end{array}$ & $\begin{array}{c}\delta \text { power } \\
(\%)\end{array}$ \\
\hline $\begin{array}{c}\text { baseline a } \\
(n=6)\end{array}$ & $96.3 \pm 1.9$ & $83.5 \pm 17.6$ & $48.6 \pm 24.1$ & $0.00 \pm 15.3$ & $0.00 \pm .0 .3$ & $25.0 \pm 8.8$ & $38.9 \pm 7.7$ \\
\hline $\mathrm{FiO}_{2} \quad 0.15 \mathrm{a}$ & $78.0 \pm 7.9^{*}$ & $79.5 \pm 10.4$ & $37.3 \pm 14.0$ & $5.2 \pm 10$ & $0.22 \pm 0.9$ & $27.1 \pm 10.0$ & $38.2 \pm 6.3$ \\
\hline $\mathrm{FiO}_{2} \quad 0.10 \mathrm{a}$ & $41.8 \pm 16.5^{\star \star}$ & $70.4 \pm 20.0$ & $40,1 \pm 16.7$ & $12.7 \pm 12.3$ & $0.26 \pm 1.1$ & $15.0 \pm 11.2$ & $46.9 \pm 6.0$ \\
\hline $\mathrm{FiO}_{2} \quad 0.05 \mathrm{a}$ & $24.1 \pm 12.9^{\star \star}$ & $53.2 \pm 16.4$ & $51.2 \pm 13.6$ & $13.6 \pm 11.6$ & $0.35 \pm 0.8$ & $9.4 \pm 5.1^{*}$ & $54.5 \pm 3.6^{*}$ \\
\hline recovery a & $94.9 \pm 3.1$ & $58.7 \pm 17.0$ & $45.4 \pm 23.2$ & $8.1 \pm 10.4$ & $-0.23 \pm 1.0$ & $19.3 \pm 5.1$ & $41.3 \pm 9.8$ \\
\hline $\begin{array}{l}\text { baseline b } \\
\quad(n=4)\end{array}$ & $94.7 \pm 0.6$ & $74.5 \pm 4.3$ & $53.9 \pm 19.6$ & $0.0 \pm 23.4$ & $0.00 \pm .0 .5$ & $22.0 \pm 3.0$ & $37.9 \pm 4.2$ \\
\hline $\mathrm{FiO}_{2} \quad 0.15 \mathrm{~b}$ & $82.2 \pm 4.2^{*}$ & $71.6 \pm 6.4$ & $56.87 \pm 24.7$ & $1.4 \pm 1.9$ & $0.55 \pm .0 .2$ & $23.0 . \pm 4.9$ & $37.3 \pm 6.4$ \\
\hline $\mathrm{FiO}_{2} 0.10 \mathrm{~b}$ & $43.3 \pm 17.1^{*}$ & $58.2 \pm 24.6$ & $25.1 \pm 14.6$ & $4.7 \pm 12.6$ & $0.91 \pm 0.6$ & $13.2 \pm 6.4$ & $51.1 \pm 8.4$ \\
\hline $\mathrm{FiO}_{2} 0.05 \mathrm{~b}$ & $23.5 \pm 6.0^{* \star}$ & $47.8 \pm 13.8$ & $32.7 \pm 13.5$ & $8.5 \pm 13.4$ & $0.77 \pm 0.9$ & $4.7 \pm 5.1^{*}$ & $52.7 \pm 9.1^{*}$ \\
\hline recovery b & $65.0 \pm 39.5$ & $30.7 \pm 28.2$ & $23.6 \pm 20.6$ & $-11.3 \pm 7.7(n=2)$ & $-0.23 \pm 0.7 \quad(n=2)$ & $10.6 \pm 14.1(n=2)$ & $43.2 \pm 12.9(n=2)$ \\
\hline
\end{tabular}

SaO2: intra-arterial oxygen saturation; MAP: mean arterial blood pressure; Qcar: carotid blood flow, ECBA: maximal mean electrocortical brain activity; Delta ( $\delta$ ) wave frequency percentage; tHb: total $\mathrm{Hb}$; Cytaa3: oxidized cytochrome aa3. ${ }^{*} \mathrm{p}<0.05$ and ${ }^{* *} \mathrm{p}<0.005$ according to non-parametric Wilcoxon matched-pairs signed-rank test. $\mathrm{n}$ : number of experiments. Data were not analyzed. Mean \pm SD. 


\section{Discussion}

We evaluated the progression of changes in cerebral electrocortical activity after repeated hypoxemic episodes in newborn piglets. The results show that normotensive, non-acidotic, normocarbic, graded and repetitive hypoxemia induces electrocortical hypofunction, whilst oxygen supply remained sufficient, assuring neuronal integrity.

In physiological conditions, cerebral blood flow (CBF) autoregulation maintains an adequate blood supply over a certain range of cerebral perfusion pressure (Volpe, 1991). During hypoxemia, neuronal oxygen consumption is assured by an increased oxygen delivery $\left(\mathrm{DO}_{2}\right)$ through increased cerebral perfusion and increased oxygen extraction $\left(\mathrm{VO}_{2}\right)$. However, cerebral perfusion is affected when severe systemic hypotension is superimposed (Johnston et al., 2001). Subsequently, both $\mathrm{DO}_{2}$ and $\mathrm{VO}_{2}$ decline below a critical level. Our previous studies tested the effect of hypoxia-ischemia (Bunt et al., 1996) and the impact of normoxic haemorrhagic hypotension on electrocortical brain activity (ECBA) (Gavilanes et al., 2001). This report describes the impact of a range of hypoxemic levels on ECBA. Hypoxemia, as part of severe acute neonatal respiratory failure, is a common feature in neonatal intensive care. Hypoxemia due to intrapulmonary shunting is related to parenchymal pulmonary disease (e.g., respiratory-distress syndrome and meconium aspiration syndrome) and to persistent pulmonary hypertension of the neonate. Less frequently, extrapulmonary shunting (e.g., congenital heart diseases and vascular diseases) implicates acute or chronic hypoxemia. Both conditions however do not necessarily cause a global hypoxia. Strategies to improve gas exchange are aimed at preventing hypoxia and hypoventilation but may also contribute to lung injury and secondary oxygen-dependent chronic lung pathology. A considerable number of NICU patients suffer from isolated (normocarbic and normotensive) hypoxic hypoxemia. In the animal model reported here, we have tried to mimic this acute neonatal isolated hypoxemia in order to study its mechanism of action.

\subsection{Characteristics of the model}

The piglet model used was non-acidotic, normocarbic, normoglycemic and normotensive. The only factor, which affected ECBA, was hypoxemia. $\mathrm{SaO}_{2}$ had to decline to $25 \%$ of its baseline value before the EEG parameters were significantly reduced. Further, MAP remained above $40 \mathrm{mmHg}$ during both hypoxemic insults.

Neuronal hypofunction, as confirmed by low amplitude EEG and increased $\delta$ power, was induced by oxygen-restricted metabolism, although without reaching critical hypoxic levels nor irreversible neuronal injury. This conclusion is supported by a sufficient cell oxygenation (Cytaa3) and the reversibility of the EEG parameters by normoxemia. We assume that the threshold of cellular oxygen deficits is lower in our model than the $\mathrm{P}_{\mathrm{a}} \mathrm{O}_{2}<5.3 \mathrm{kPa}$ proposed by Torrance and Wittnich. In contrast to our results, their hypoxic 3-days-old piglet model showed lactic acidemia at $\mathrm{P}_{\mathrm{a}} \mathrm{O}_{2}$ values below $5.3 \mathrm{kPa}$ (Torrance and Wittnich, 1994).

Anaesthesia was achieved with halothane. This gas increases slow-wave EEG power and the dominant frequency prior to anaesthesia (Wauquier, 1993). In addition, our experiments were started after one-hour stabilization period under a 
constant dose of anaesthetic and only when no further changes in EEG amplitude or spectral power were seen.

Finally, $Q_{\text {car }}$ represents CBF. Meadow et al. (1999) demonstrated that a $Q_{\text {car }}$ signal from the common carotid artery corresponded to CBF as determined by radioactive microspheres in a hypercarbic and hypoxic newborn piglet model.

\subsection{MCM and conventional EEG}

Continuous recording of traditional multi-channel EEG identifies clinical and subclinical seizure activity and enables the assessment of background activity (amplitude and frequency spectra) during physiological and pathological states. Additionally, EEG background activity could be correlated to long-term neurological outcome. It has been demonstrated that the EEG parameters depicted by the MCM correspond with that of conventional EEG (Peck et al., 1981; Thiringer et al., 1986; Hellström-Westas, 1992; Greisen, 1994; al Naqeeb et al., 1999). Though, conventional EEG offers a greater reliability when diagnosing short subclinical seizures (Selton D and Andre M., 1997).

\subsection{MCM amplitude and power analysis}

MCM amplitude patterns have been described in normal premature and full-term infants (Verma et al., 1984; Viniker et al., 1984; Thornberg and Thiringer, 1990; Hellström-Westas et al., 1991), and in several pathological conditions, including it's use as a predictor of neurological outcome after hypoxic-ischemic encephalopathy in full-term infants (Toet et al., 1999). Furthermore, since its first report by Maynard in 1979, EEG frequency analysis in experimental studies of fetal or newborn animals have been_scarce (Keunen et al., 2000; Gavilanes et al., 2001). Spectral power in the $\delta$ band was analyzed for several reasons. First, the neonatal basic frequency spectrum ranges between 3 to $4 \mathrm{~Hz}$. Additionally, inhalational anaesthetics increase slow-wave EEG activity (Wauquier, 1993). This influence could explain the dominance of $\delta$ activity as well as the decrease of variability of the other waves throughout the experiment. Finally, an increase in $\delta$ wave activity has been reported during post-hypoxic encephalopathy (Fisch, 1991). In this work, $\delta$ frequency percentage increased markedly during severe hypoxemia $(p<0.05)$ and recovered to baseline values during reoxygenation $\left(\mathrm{FiO}_{2} 0.21\right)$.

\subsection{Near infrared spectroscopy (NIRS)}

The NIRS-Critikon software calculates regional changes in optical absorption of near infrared light and converts these into changes in the redox state of haemoglobin and Cytaa3, providing a noninvasive monitoring technique of the delivery and utilization of oxygen to and by the brain tissue (Siebenthal and Casaer, 1992). Data of several studies have validated and documented the value of NIRS in specific experimental and clinical situations. Tsuji et al. (1995), and more recently Chang et al. (1999) showed a significant decrease in Cytaa3 with a concomitant loss of cerebral energy during combined hypoxia and ischemia in the newborn piglet. Shadid et al. (1999) found an increase of Cytaa3 during hypercapnic hypoxia, followed by a significant decrease during haemorrhagic hypotension. Nomura et al. (1996) documented a decreased Cytaa3 during hypothermic 
cardiopulmonary bypass in 1 to 2 week old piglets. Hypothermic cardiopulmonary bypass in infants less than 11 months old also showed similar patterns of Cytaa3 changes (van Bel et al., 1996). Finally, CBV and Cytaa3 were impaired in the early postasphyctic period in neonates of $>34$ weeks gestational age (van Bel et al., 1993).

The combination of EEG amplitude, but not power analysis, and Cytaa3 monitoring has been previously reported. Marks et al. (1996) showed that ECBA was depressed and Cytaa3 was decreased during and after 30 min of bilateral carotid occlusion in ischemic fetal sheep. Finally, EEG changes occurred at a higher level of oxygenation (FiO2 0.15) than those at which Cytaa3 fell (FiO2 0.10) in an hypoxic adult rat model (Hoshi et al., 1997). These data all support the correlation between the cellular oxygen state with the redox state of Cytaa3. However, the latter is also influenced by several biochemical changes. Cytaa3 alterations could represent the metabolic effects of $\mathrm{P}_{\mathrm{a}} \mathrm{CO}_{2}$ through intracellular $\mathrm{pH}$, energy compounds and nitric oxide (NO), rather than an increased oxygen delivery (Edwards et al., 1991; Quaresima et al., 1998). The vascular role of NO, a diffusible free radical synthesized under the influence of cytosolic calcium in endothelial cells and neurons, is to coordinate perfusion by vasodilation and to modulate neuronal activity. During hypoxic-ischemic encephalopathy, NO production is pathologically increased and $\mathrm{NO}$ binds to the oxygen binding site of Cytaa3, inducing a reversible inhibition of Cytaa3. To the contrary, more severe asphyctic insults result in higher NO levels and with an irreversible inhibition of Cytaa3 and cell respiration by peroxynitrites (Brown, 1999). In this study, $\mathrm{tHb}$ as an index of CBV did not change during hypoxemia as compared to baseline, neither did Cytaa3. Both NIRS measurements had the tendency toward an augmentation during hypoxemia, yet this effect had no statistical significance. The lack of changes in cerebral circulation and neuronal oxygenation could be explained by the sufficient cerebral perfusion being supplied by arterial tension and normocarbia. In contrast to the above studies, our model induced isolated hypoxemia. NIRS showed an appropriate cellular oxygenation pattern, which paralleled ECBA hypofunction during both of the $0.05 \mathrm{FiO}_{2}$ hypoxemic phases.

In summary, this paper reports for the first time MCM relative spectral $\delta$ power and NIRS changes in a newborn model of isolated hypoxemia in the piglet. Our data showed that isolated, graded and repetitive hypoxemia induces electrocortical hypofunction whilst maintaining cellular oxygenation. These data support our primary statement that continuous monitoring of cerebral electrical activity is relevant during routine intensive neonatal care. 


\section{Acknowledgments}

The authors thank T. van der Nagel, Laboratory of Physiology, and D. van Mierlo, Department of Clinical Neurophysiology, University Hospital of Maastricht for their technical assistance. 


\section{References}

al Naqeeb N, Edwards AD, Cowan FM, Azzopardi D. Assessment of neonatal encephalopathy by amplitude-integrated electroencephalography. Pediatrics 1999;103(6):1263-71.

Bunt JEH,Gavilanes AWD, Reulen JPH, Blanco CE, Vles JSH. The Influence of Acute Hypoxemia and Hypovolemic Hypotension of Neuronal Brain Activity Measured by the Cerebral Function Monitor in Newborn Piglets. Neuropediatrics 1996;27(5):260-264.

Brown GC . Nitric oxide and mitochondrial respiration. Biochimica et Biophysica Acta 1999;1411:351-369.

Chang YS, Park WS, Lee M, Kim KS, Shin SM, Choi JH. Near infrared spectroscopic monitoring of secondary cerebral energy failure after transient global hypoxia-ischemia in the newborn piglet. Neurol Res. 1999;21(2):216-24.

Delpy DT, Cope M, van der Zee P,Arridge SR, Wray SC, Wyatt JS. Estimation of optical pathlength through tissue by direct time of flight measurement. Phys. Med. Biol. 1988;33:1433-1442.

Edwards AD, Brown, G.C., Cope, M., Wyatt, J.S., McCormick, D.C., Roth, S.C., Delpy, D.T. and Reynolds, E.O.R. Quantification of concentration changes in neonatal human cerebral oxidised cytochrome oxidase. J. Appl. Physiol. 1991; 71(5): 1907-13.

Fisch BJ. Bilaterally synchronous slow waves. In: Fisch BJ. Spehlmanss's EEG primer. 2nd ed. Elsevier Science B.V., Amsterdam, The Netherlands, 1991: 441458.

Gavilanes AWD, J.Vles, R.Van Sprundell, K.Von Siebenthal, F.Nieman, J.Reulen, C.Blanco. Electrocortical brain activity, cerebral haemodynamics and oxygenation during progressive hypotension. Clin Neurophysiology 2001;112(1):52-59.

Greisen G. Tape-recorded EEG and the cerebral function monitor: amplitudeintegrated, time-compressed EEG. J.Perinat.Med.1994;22:541-546.

Hellström-Westas L, Rosén I, Svenningsen NW. Cerebral function monitoring during the first week of life in extremely mall low birthweight (ESLBW) infants. Neuropediatrics 1991;22:27-32.

Hellström-Westas L. Comparison between tape-recorded and amplitude-integrated EEG monitoring in sick newborn infants. Acta Paediatr.Scand.1992;81:812-819 
Hoshi Y, Hazeki O, Kakihana Y, Tamura M. Redox behavior of cytochrome oxidase in the rat brain measured by near-infrared spectroscopy. J.Appl.Physiol. 1997;83(6):1842-8.

Johnston MV, Trescher WH, Ishida A, Nakajima W. Neurobiology of hypoxicischemic injury in the developing brain. Pediatr.Res. 2001; 49(6): 735-41.

Keunen H, JSH Vles, JLH van Reempst, AWD Gavilanes, THM Hasaart.. The effect of repetitive umbilical cord occlusions on neuronal brain activity measured by the Cerebral function Analysing Monitor and histological outcome in the imature fetal sheep. J Soc Gynecol Investig $2000 ; 7(4): 218-23$.

Maynard DE. Development of the CFM: The cerebral function analysing monitor (CFAM). Ann.Anesth.Franc.1979;3:253.

Marks KA, Mallard EC, Roberts I, Williams CE, Sirimanne ES, Johnston B, Gluckman PD, Edwards AD. Delayed vasodilation and altered oxygenation after cerebral ischemia in fetal sheep. Pediatr.Res. 1996;39(1):48-54.

Meadow W, Rudinsky B, Raju T, John E, Fornell L, Shankararao R.. Correlation of flow probe determinations of common carotid artery blood flow and internal carotid artery blood flow with microsphere determinations of cerebral blood flow in piglets. Pediatr.Res. 1999;45:324-330.

Nomura F, Naruse , du Plessis A, Hiramatsu T, Forbess J, Holtzman D, VolpeJJ, Jonas R, Tsuji M. Cerebral Oxygenation Measured by Near Infrared Spectroscopy during Cardiopulmonary Bypass and Deep Hypothermic Circulatory Arrest in Piglets. Pediatr.Res.1996;40:790-796.

Peck Mc M RRT, Seriff NS, Wiener V, MD. Comparison of the cerebral function monitor with the EEG in determining brain death. Critical Care Medicine 1981;9 (6):459-463.

Quaresima V, Springett R, Cope M, Wyatt JT, Delpy DT, Ferrari M, Cooper CE. Oxidation and reduction of cytochrome oxidase in the neonatal brain observed by in vivo near-infrared spectroscopy. Biochim.Biophys.Acta 1998;1366(3):291-300.

Reulen JPH, Gavilanes AWD, van Mierlo D, Blanco CE, Spaans F, Vles JSH. The Maastricht Cerebral Monitor (MCM) for the neonatal intensive care unit. Journal of Medical Engineering \& Technology 1999; 23(1):29-37.

Rothman SM. Biochemistry of Hypoxic-Ischemic Brain Injury. In: Fetal and Neonatal Physiology. Polin RA. And Fox WW. (eds) $2^{\text {nd }}$ ed. WB Saunders, Philadelphia, PA, 1998;pp 2118-2122. 
Selton D and Andre M. Prognosis of hypoxic-ischaemic encephalopathy in full-term newborns--value of neonatal electroencephalography. Neuropediatrics 1997; 28(5): 276-80.

Shadid M, Hiltermann L, Monteiro L, Fontijn J, Van Bel F. Near infrared spectroscopy-measured changes in cerebral blood volume and cytochrome aa3 in newborn lambs exposed to hypoxia and hypercapnia, and ischemia: a comparison with changes in brain perfusion and O2 metabolism. Early Hum.Dev.1999 ;55(2):169-82.

Siebenthal von K, Casaer GBP. Near-infrared spectroscopy in newborn infants. Brain Dev.1992;14:135-143.

Thiringer KJ, Connel R, Oozeer E, Carter M. Levene: Comparison between continuous Medilog EEG and Cerebral Function Monitor recordings on infants in neonatal intensive care. Early Hum.Develop.1986;14:150-151.

Thornberg E, Thiringer K. Normal pattern of the cerebral function monitor trace in term and preterm neonates. Acta Paediatr.Scand.1990;79:20-25.

Toet MC, Hellstrom-Westas L, Groenendaal F, Eken P, de Vries LS. Amplitude integrated EEG 3 and 6 hours after birth in full term neonates with hypoxicischaemic encephalopathy. Arch.Dis.Child.Fetal Neonatal Ed.1999;81(1):F19-F23.

Torrance SM, Wittnich C Blood lactate and acid-base balance in graded neonatal hypoxia: evidence for oxygen-restricted metabolism. J.Appl.Physiol.1994; 77(5):2318-24.

Tsuji M, Naruse H, Volpe J, Holtzman D. Reduction of Cytochrome aa3 ured by Near-Infrared Spectroscopy Predicts Cerebral Energy Loss in Hypoxic Piglets. Pediatr.Res.1995;37:253-259.

van Bel F, Dorrepaal CA, Benders MJ, Zeeuwe PE, van de Bor M, Berger HM. Changes in cerebral hemodynamics and oxygenation in the first 24 hours after birth asphyxia. Pediatrics 1993; 92(3):365-72.

van Bel F, Zeeuwe PE, Dorrepaal CA, Benders MJ, Van de Bor M, Hardjowijono R. Changes in cerebral hemodynamics and oxygenation during hypothermic cardiopulmonary bypass in neonates and infants. Biol.Neonate 1996;70(3):141-54.

Verma UL, Archbald F, Tejani NA, Handwerker SM. Cerebral function monitor in the neonate. I: Normal patterns. Dev.Med.Child.Neurol. 1984;26:154-161.

Viniker DA, Maynard DE, Scott DF. Cerebral function monitor studies in neonates. Clin.Electroencephalogr.1984;15:185-192. 
Volpe J. Hypoxic-ischemic encephalopathy: Biochemical and Physiological aspects. In: Volpe J. Neurology of the newborn 4th ed. WB Saunders, Philadelphia, PA, 2001: 217-276.

Wauquier A. EEG and neuropharmacology. In: Niedermeyer E. and Lopes da Silva F. Electroencephalography. Basic principles, clinical applications, and related fields. 3th ed. Williams \& Wilkins, 1993: 619-629. 
CHAPTER 8.

\section{ELECTRICAL BRAIN ACTIVITY DURING NORMOTENSIVE HYPOXEMIA IN VERY LOW BIRTH WEIGHT INFANTS}

AWD Gavilanes, JSH Vles, M.van Reisen, VHJM van Kranen-Mastenbroek, JPH Reulen and CE Blanco.

Neuropediatrics, accepted 


\begin{abstract}
The aim of the study was to determine whether acute isolated hypoxemic events could cause simultaneous neuronal dysfunction in the preterm brain during the immediately postnatal period. Electrical brain activity was monitored with an amplitude-integrated quantitative electroencephalogram called the Maastricht cerebral monitor (MCM), from 12 up to 72 hours postnatally in fourteen very low birth weight infants (VLBWI: $<1500 \mathrm{~g}$ birth weight). The last five min of each hypoxemic episode (arterial oxygen saturation $\left[\mathrm{SaO}_{2}\right]<0.86$ ) were compared with the last five min of the previous normoxic period $\left(\mathrm{SaO}_{2}>0.86\right.$, baseline) and with the first five min of the normoxic recovery.

Eight VLBWI (57\% of eligible; gestational age: 24 to 31 weeks; birth weight: 575 $1470 \mathrm{~g})$ exhibited 66 episodes of isolated hypoxemia $\left(\mathrm{SaO}_{2} 0.85\right.$ to 0.67$)$. MCM amplitude analysis during hypoxemia, relative spectral power, raw EEG and MCM amplitude patterns were comparable to baseline and recovery values. Normotensive preterm infants without encephalopathy maintained an unchanged electrical brain activity under isolated moderate acute hypoxemia. These data suggest that the limit of hypoxemia used in clinical medicine $\left(\mathrm{SaO}_{2}\right.$ of 0.85$)$ is significant higher than the neuronal hypoxic threshold. At that given level, adaptive response to hypoxemia and neuronal oxygen extraction assures an appropriate oxygen metabolism.
\end{abstract}

\title{
Key words
}

Maastricht Cerebral Monitor - Electroencephalogram - Hypoxemia - Hypoxia Very low birth weight infants - NICU

\section{Abbreviations}

$\begin{array}{lll}\text { aEEG } & : & \text { amplitude-integrated electroencephalogram } \\ \mathrm{CFM} & : & \text { cerebral function monitor } \\ \mathrm{CBF} & : & \text { cerebral blood flow } \\ \mathrm{EEG} & : & \text { electroencephalogram } \\ \mathrm{MCM} & : & \text { Maastricht cerebral monitor } \\ \mathrm{NICU} & : & \text { neonatal intensive care unit } \\ \mathrm{SaO}_{2} & : & \text { transcutaneous arterial oxygen saturation } \\ \mathrm{VLBWI} & : & \text { very low birth weight infants }\end{array}$

\section{Introduction}

Severely ill preterm newborn infants, requiring respiratory and circulatory support, are commonly exposed to acute, short and repetitive hypoxemic insults. Potentially, these insults can cause brain injury and long-term neurodevelopmental disabilities. Monitoring electrical brain activity could therefore help to identify those infants with neuronal dysfunction and those with seizure activity, helping to establish a prompt clinical intervention. However, the logistics necessary to arrange and to perform a multichannel conventional electroencephalogram (EEG) and the complexity of its interpretation have reduced considerably its implementation as an on-line 
monitoring tool in the neonatal care. By contrast, amplitude-integrated EEG (aEEG) using a one-channel biparietal EEG lead cerebral function monitor (CFM) has become a standard in some neonatal units, dealing traditionally with full-term postasphyctic infants rather than non-asphyctic extremely preterm infants.

Literature is scarce about electrical brain activity in very low birth weight infants (VLBWI) depicted by EEG as well as by aEEG and it mainly involved infants older than 28 weeks gestation $(2,16)$. Furthermore, the relationship between acute hypoxic hypoxia and electrical brain activity has not been evaluated in VLBWI.

In the past years, we performed a series of fetal and neonatal animal experiments using the Maastricht cerebral monitor (MCM), a three-channel computerized quantitative aEEG registration $(9,11,21,24)$. After completing its development and testing its reliability, we implemented MCM clinically. In particular, the study of graded hypoxemia in a normotensive, non-acidotic newborn piglet model lend the basis for the present study (12). That study showed that only severe isolated graded hypoxemia led to acute changes in neuronal activity, whereas cellular oxygenation remained unaffected. In this animal model, a $\mathrm{SaO}_{2}$ under $0.20\left(\mathrm{P}_{\mathrm{a}} \mathrm{O}_{2} 2.68 \pm 1.08\right.$ $\mathrm{kPa}$ ) was necessary to obtain significant MCM amplitude and spectral changes. Although the experimental setting mimicked the clinical situation, MCM changes were induced in the full-term anaesthetized piglet brain. Therefore, we conducted the present study in order to investigate whether acute hypoxemic events would cause electrical brain changes in non-anesthetized VLBWI without encephalopathy. We expected EEG changes in terms of electrical hypofunction or dysfunction during hypoxemia, and their normalization during normoxemic recovery.

\section{Patients and methods}

We prospectively studied fourteen VLBWI born between 24 and 31 weeks gestational age (GA), admitted to the neonatal intensive care unit (NICU) at the University Hospital of Maastricht over an 18-month period. Oral parental consent was obtained from both parents. We included in this study VLBWI with normal parenchymal cranial ultrasound scans (US), without clinical signs of perinatal hypoxic ischemic encephalopathy (HIE) (umbilical artery $\mathrm{pH}>7.0$ ) or other encephalopathies. Continuous time synchronized data included heart rate, mean arterial blood pressure and postductal $\mathrm{SaO}_{2}$ measurements (Hewlett-Packard Neonatal Component Monitoring System, Palo Alto, CA, USA). Our policy with regard to normal $\mathrm{SaO}_{2}$ settings in preterm infants established a lower limit of 0.86 and an upper limit of 0.93. Therefore, hypoxemia was defined as $\mathrm{a} \mathrm{SaO}_{2}$ of less than or equal to $0.85(29)$.

\section{MCM registration}

The MCM system has been previously described by Reulen et al.(24). It plotted and computed the weighted EEG amplitude distribution (maximum mean, minimum mean , and bandwidth mean) in $\mu \mathrm{V}$, and relative spectral power (delta $(\delta)$ [1-3.5 $\mathrm{Hz}$ ], theta $(\theta)$ [3.5-7.5 Hz], alpha $(\alpha)[7.5-13 \mathrm{~Hz}]$ and beta $(\beta)[13-27 \mathrm{~Hz}])$ in every two-second epoch. Electrical brain activity was recorded using the MCM from three channels: $P_{3}-F_{z}, P_{4}-F_{z}$ and $P_{3}-P_{4}$, according to the 10-20 international system. Electrodes were applied on the scalp using electrode paste and were fixed with collodium. The resistance was $<2 \mathrm{k} \Omega$ for all electrodes. Monitoring was continuous 
for periods of up to three days, starting within the first 12 hours of life after parental consent had been obtained.

MCM analysis

Off-line quantitative amplitude and relative spectral analysis of the last five minutes of each hypoxemic episode $\left(\mathrm{SaO}_{2} 0.85\right.$ to 0.67$)$ were compared to the last five minutes of the previous normoxic period (baseline) and with the first five minutes of the normoxic recovery.

MCM pattern recognition

Five minutes baseline, hypoxemia and recovery raw EEG's and aEEG's from each patient were visually inspected and compared for continuity or discontinuity, and for low or normal voltage. Features taking into account in visual inspection were the suppression duration, burst activity and burst amplitude.

Satistics

Statistical analysis was done by the repeated measurements analysis of variance ANOVA, significance at $p<0.05$. Analysis was carried out using SPSS-10.0 (SPSS Inc., Chicago, Illinois, USA).

\section{Results}

Fourteen VLBWI were eligible for MCM registration (GA: 24 to 31 weeks; birth weight: $575-1470 \mathrm{~g}$ ). The mean duration of the registration was 40 hours (24 to 72 hours). Eight out of the fourteen patients exhibited episodes of isolated hypoxemia $\left(\mathrm{SaO}_{2}<0.86\right)$ for 5 to 15 minutes duration. Table 1 summarizes the clinical data of those infants. Six patients of the study group needed artificial ventilation and invasive blood pressure monitoring. Nasal continuous positive airway pressure was needed in two other infants. All mechanically ventilated patients received narcotics type fentanyl ( 1 to $2 \mu \mathrm{g} / \mathrm{kg} / \mathrm{hr}$ ). One of those patients was receiving dopamine 5 $\mu \mathrm{g} / \mathrm{kg} / \mathrm{min}$ and remained haemodinamic stable during the study period. Intraventricular hemorrhage grade I following Volpe (28) was detected in three patients. None of the infants presented ultrasonographic signs of brain parenchymal pathology during the study period.

A total of 66 hypoxemic episodes (2 to 17 episodes per patient) were registered. $\mathrm{SaO}_{2}$ varied between 0.67 and 0.85 during the analyzed 5 minutes period at the end of the hypoxemic event. $\mathrm{P}_{3}-\mathrm{P}_{4} \mathrm{MCM}$ amplitude did not change significantly between the study periods: baseline (maximum mean $6.7 \pm 3.1 \mu \mathrm{V}$, minimum mean $2.4 \pm 1.8 \mu \mathrm{V}$ and bandwidth $4.5 \pm 2.4 \mu \mathrm{V}$ ), hypoxemia (maximum mean $7.1 \pm 5.0 \mu \mathrm{V}$, minimum mean $2.9 \pm 3.4 \mu \mathrm{V}$ and bandwidth $4.9 \pm 4.2 \mu \mathrm{V}$ ), recovery (maximum mean $6.9 \pm 3.2 \mu \mathrm{V}$, minimum mean $2.6 \pm 2.8 \mu \mathrm{V}$ and bandwidth $4.8 \pm 2.9 \mu \mathrm{V}$ ). Table 2 summarizes these MCM characteristics. There were no significant effects of hypoxemia on the relative spectral power data: baseline $(\delta 48.2 \pm 12.6 \%, \theta$ $28.9 \pm 13.5 \%, \alpha 14.5 \pm 3.5 \%$ and $\beta 8.2 \pm 1.9 \%)$, hypoxemia $(\delta 51.8 \pm 12.0 \%, \theta$ $27.6 \pm 11.9 \%, \alpha 12.7 \pm 2.0 \%$ and $\beta 7.7 \pm 2.1 \%)$ and recovery $(\delta 47.0 \pm 12.5 \%, \theta$ $30.1 \pm 14.6 \%, \alpha 13.9 \pm 2.8 \%$ and $\beta 9.2 \pm 2.3 \%$ ).

Visual inspection of the raw EEG and MCM amplitude patterns showed appropriate background activity for GA, which was predominantly discontinuous. 
Differences between the 3 study periods were not quantified. No seizure activity was detected in any patient at the time of the study. As a typical illustration, figure 1 demonstrates the MCM amplitude patterns $\left(\mathrm{P}_{3}-\mathrm{P}_{4}\right)$ during baseline, hypoxemia and recovery in patient no. 2 (GA $251 / 7$ weeks).

Table 1. Characteristics of the infants presenting hypoxemic periods $(n=8)$

\begin{tabular}{|c|l|l|l|l|l|l|}
\hline $\begin{array}{l}\text { patient } \\
\text { no. }\end{array}$ & $\begin{array}{c}\text { GA } \\
\text { (wks) }\end{array}$ & $\begin{array}{c}\text { BW } \\
\text { (percentile) }\end{array}$ & UApH & $\begin{array}{l}\text { clinical } \\
\text { diagnose }\end{array}$ & $\begin{array}{l}\text { ventilation } \\
\text { therapy }\end{array}$ & $\begin{array}{l}\text { hypoxemic } \\
\text { periods (n) }\end{array}$ \\
\hline $\mathbf{1}$ & $240 / 7$ & $760(10-50)$ & 7.17 & RDS grade 4 & MV & 11 \\
\hline $\mathbf{2}$ & $251 / 7$ & $730(10)$ & 7.11 & RDS grade 2 & n-CPAP & 8 \\
\hline $\mathbf{3}$ & $252 / 7$ & $780(10)$ & 7.07 & RDS grade 3 & MV & 2 \\
\hline $\mathbf{4}$ & $253 / 7$ & $730(2.3-10)$ & 7.05 & RDS grade 3 & MV & 3 \\
\hline $\mathbf{5}$ & $260 / 7$ & $575(2.3)$ & 7.19 & RDS grade 3 & MV & 17 \\
\hline $\mathbf{6}$ & $275 / 7$ & $710(2.3-10)$ & 7.10 & RDS grade 3 & MV & 7 \\
\hline $\mathbf{7}$ & $286 / 7$ & $970(10)$ & 7.03 & RDS grade 3 & MV & 14 \\
\hline $\mathbf{8}$ & $310 / 7$ & $1470(10-50)$ & n.a. & RDS grade 2 & n-CPAP & 4 \\
\hline
\end{tabular}

$\mathrm{GA}=$ gestational age in weeks; $\mathrm{BW}=$ birth weight in grams; $\mathrm{UapH}=$ umbilical artery $\mathrm{pH}$; n.a.= not available; $\mathrm{MV}=$ mechanical ventilation; $\mathrm{n}-\mathrm{CPAP}=$ nasal $\mathrm{CPAP} ; \mathrm{RDS}=$ respiratory distress syndrome.

Table 2. Comparison of computed MCM parameters during acute hypoxemia in $\operatorname{VLBWI}(n=8)$

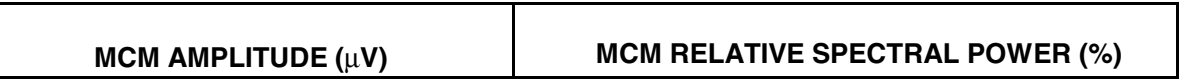

\begin{tabular}{|l|l|l|l|l|l|l|l|}
\hline PERIOD & MAXIMUM & MINIMUM & $\begin{array}{l}\text { BAND } \\
\text { WIDTH }\end{array}$ & DELTA & THETA & ALPHA & BETA \\
\hline BASELINE & $6.7 \pm 3.1$ & $2.4 \pm 1.8$ & $4.5 \pm 2.4$ & $48.2 \pm 12.6$ & $29.0 \pm 13.5$ & $14.5 \pm 3.5$ & $8.2 \pm 1.9$ \\
\hline $\begin{array}{l}\mathrm{SaO}_{2} \\
(\mathbf{0 . 8 5}-\mathbf{0 . 6 7 )}\end{array}$ & $7.1 \pm 5.0$ & $2.9 \pm 3.4$ & $4.9 \pm 4.2$ & $51.8 \pm 12.0$ & $27.6 \pm 11.9$ & $12.7 \pm 2.0$ & $7.7 \pm 2.1$ \\
\hline RECOVERY & $6.9 \pm 3.2$ & $2.6 \pm 2.8$ & $4.8 \pm 2.9$ & $47.0 \pm 12.5$ & $30.1 \pm 14.6$ & $13.9 \pm 2.8$ & $9.2 \pm 2.3$ \\
\hline
\end{tabular}

ANOVA repeated measurements showed no significant differences between the three periods. Means \pm SD. 
Fig. 1.

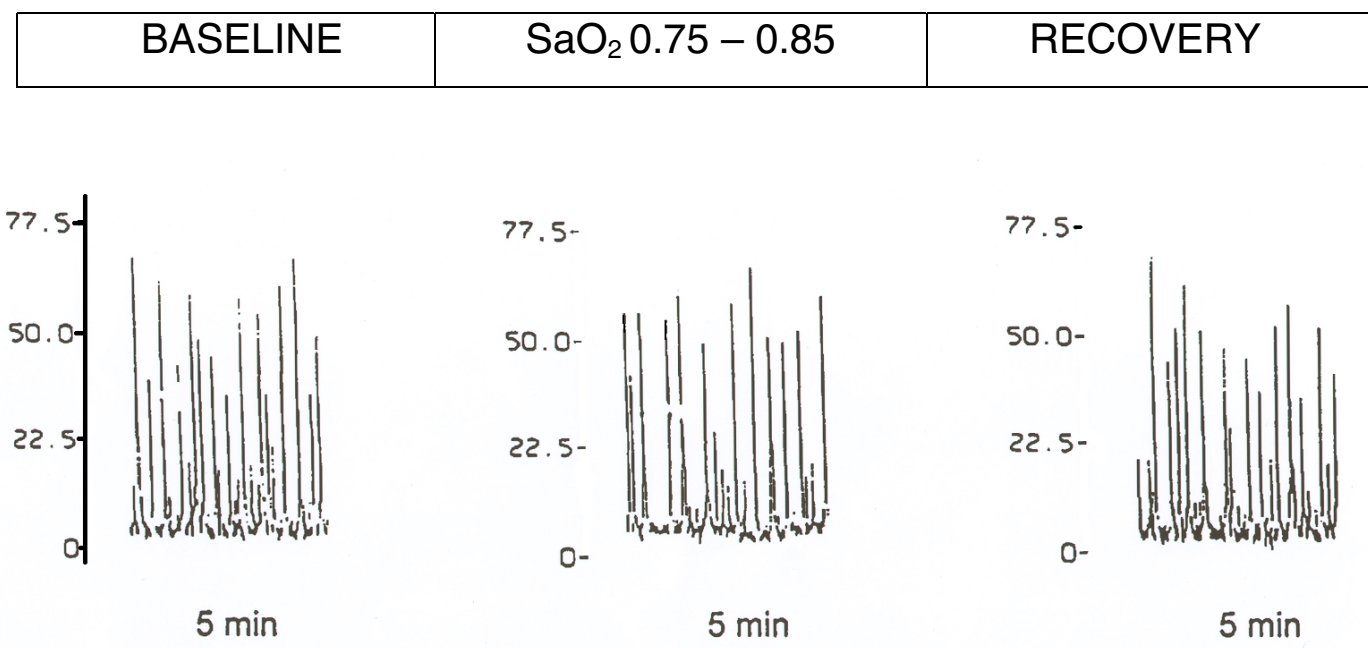

Representative example of MCM amplitude patterns in $\mu \mathrm{V}$ during baseline, hypoxemia and recovery in a $251 / 7$ weeks GA (patient no. 2). MCM amplitude patterns remained unchanged during hypoxemia $\left(\mathrm{SaO}_{2} 0.75\right.$ to 0.85$)$.

\section{Discussion}

This study was conducted to reveal further understanding of neuronal function under acute hypoxemic conditions in normotensive VLBWI. The data showed that those infants maintained an unchanged electrical brain activity under isolated acute short mild to moderate hypoxemia. We primarily expected a decrease of aEEG amplitude and a shift of the power spectrum to lower frequencies in response to the hypoxemic insult. However, neither aEEG amplitude nor spectral power were influenced by hypoxemia. In this manner, the present data open several points of discussion.

The traditional multichannel EEG registration enables detection of electrical brain activity abnormalities, which are strictly linked to abnormal cerebral metabolism, haemodynamics or oxygenation. These abnormalities may remain clinically unrecognized because of sedation, muscle paralysis or electro-clinical dissociation in case of seizure activity in newborn infants. EEG monitoring depicts actual neuronal function and therefore enables early identification of abnormalities. This might be crucial in the neonatal intensive care because of the increased number of survivors with permanent neurological sequel (4). Based on the well established diagnostic and prognostic value of background full-term neonatal EEG activity (20), the classical single-channel aEEG called CFM has been validated and has emerged as a reliable diagnostic tool $(1,7)$. Clinical neonatal studies using CFM mainly focussed on the recognition of abnormal patterns in term infants, e.g., isoelectric or burst-suppression patterns. These studies showed that single-channel 
aEEG abnormal patterns are strongly associated with permanent neurologic deficit $(6,10)$. However, neonatal intensive care deals not only with full-term asphyctic infants, but essentially with extremely preterm infants. The analysis of the latter is more complex because age-appropriate maturational EEG and aEEG patterns of extreme preterm infants are not yet well studied, and information about abnormal aEEG patterns of discontinuous physiological background EEG is exceptional.

There have been a number of studies in recent years attempting to define a normal reference range of background characteristics using early ( $<24 \mathrm{~h}$ of age) multichannel EEG in extreme preterm infants. Biagioni et al. (5) presented the socalled occipital sawtooth as a physiological EEG pattern characteristic of preterm infants between 24-26 weeks GA. Selton et al. (26) described EEG patterns of normal preterm infants between 26 and 28 weeks GA and Hayakawa et al. (14) showed the characteristics of the normal background EEG in newborns from 21 weeks through 26 weeks of gestation. Those patterns are basically interhemispherically synchronous and the percentage of discontinuous EEG activity correlates inversely with GA. In contrast, little has been reported regarding aEEG in VLBWI. Early short-term single-channel aEEG in VLBWI has been used to characterize changes in EEG background activity after perinatal asphyxia (3) and during the administration of surfactant (22). In addition, early long-term (continuos) single channel aEEG has been applied in VLBWI to detect subclinical seizures (15), to estimate outcome in infants with or without perinatal HIE $(7,16)$, to evaluate EEG changes during surfactant treatment (17) and to monitor the effect of anticonvulsant drugs (18). This is the first study to report measurements of electrical brain activity changes in normal preterm brain during hypoxemia registered by early long-term aEEG.

With the development of the three-channel Maastricht cerebral monitor (MCM) (24), we were able to disclose information not available from visual assessment of the classical one-channel aEEG paper tracing e.g., the on-and off-line display of original raw EEG and its frequency spectra. In addition, MCM enabled on-and offline signal analysis of aEEG amplitude and relative spectral power.

All our mechanically ventilated patients required continuous infusion of fentanyl as routine clinical management at 1 to $2 \mu \mathrm{g} / \mathrm{kg} / \mathrm{hr}$ (25). No protective effect to the preterm brain was expected using that low maintenance dose.

In this study, normotensive normal preterm brain preserved unchanged electrical brain activity under isolated mild to moderate acute hypoxemia. The lack of change in EEG parameters opens interesting points of debate, with respect to the hypoxemic insult and its adaptation, and secondly, to the neuronal tolerance to hypoxemia.

\section{Hypoxemia}

The most appropriate $\mathrm{SaO}_{2}$ reference range in newborns is a controversial item (23). In clinical practice, a $\mathrm{Pa} \mathrm{O}_{2}$ around $5.0 \mathrm{kPa}$ could be considered the lower edge of normoxia in preterm infants. This level of oxygen tension is considered appropriate because it is sufficient in fetal life, it assures a normal acid-base balance neonatally and it lies far above the level at which cyanosis becomes clinically evident. Wasunna et al. (29) showed that this oxygen tension level 
corresponds to $\mathrm{a} \mathrm{SaO}_{2}$ of 0.86 in preterm infants. Therefore, our ward protocols aim to keep $\mathrm{SaO}_{2}$ between the target range of 0.86 to 0.93 . Hypoxic hypoxemia defined as $a \mathrm{SaO}_{2}$ of $<0.85$ in mechanically ventilated infants (8) is related to intrapulmonary and extrapulmonary shunting, e.g., alveolar hypoventilation due to active expiration events, tube obstruction or dislocation. During the hypoxemic event, neuronal oxygen consumption is initially met by an increased oxygen delivery $\left(\mathrm{DO}_{2}\right)$ through increased cardiac output and centralization of blood flow. Another compensatory mechanism to maintain oxygen consumption is to increase oxygen extraction $\left(\mathrm{VO}_{2}\right)$ with a reduction of the venous oxygen content (19). Oxygen restricted metabolism takes place when compensatory mechanisms fail and both $\mathrm{DO}_{2}$ and $\mathrm{VO}_{2}$ decline below a critical level. The lack of EBA changes from hypoxemia in our patients may be explained by the adequacy of these compensatory mechanisms at this $\mathrm{SaO}_{2}$ level.

\section{Neuronal tolerance}

It is a clinical fact that chronic lower oxygen conditions can be well tolerated by infants of any gestational age if systemic haemodynamic and metabolic conditions are stable, e.g., infants with cyanotic cardiopathies. Neurodevelopmental studies have confirmed this clinical statement. Graziani et al. (13) showed that hypoxemia without systemic hypotension prior to or during neonatal extracorporal membrane oxygenation in (near) term newborn infants did not contribute to any neurologic or audiologic sequel at 15 months of age of older. Recently, evidence showed that preterm infants of less than 28 weeks gestation managed in a lower saturation range $\left(\mathrm{SaO}_{2} 0.70\right.$ to 0.90$)$ developed significant less retinopathy of prematurity, less respiratory support and better growth than their counterparts nursed in a richer oxygen environment $\left(\mathrm{SaO}_{2} 0.88\right.$ to 0.98$)$. Both groups of preterm infants exhibit identical incidence of cerebral palsy and survival rate at one year corrected age (27).

What is the critical level of inappropriate cellular oxygen delivery and secondary disrupted cerebral metabolism linked with long-term poor neurodevelopment? Oxygen therapy harms pulmonary, retinal and eventually brain cells. Do we overtreat when targeting $\mathrm{SaO}_{2}$ of $>0.86$ in the tiny preterm infant? Our study doesn't answer these questions, rather it opens them and shows that neuronal electrical activity and consequently neuronal oxygen metabolism were preserved during short-term isolated acute hypoxemia. These findings, however, might be only applicable to the intact preterm brain.

Altogether, the present data support the concept that the lower limit of normoxia used in clinical medicine $\left(\mathrm{SaO}_{2}\right.$ of 0.86$)$ is significantly higher than the neuronal hypoxic threshold in the non-encephalopatic preterm brain. Additional (electrophysiological) studies are needed regarding the eventual implementation of a more restrictive oxygen supply policy in preterm infants.

\section{Acknowledgments}

Thanks are due to the nurse-staff of the NICU and to Katrien Boots, medical student, for assistance with data collection. 


\section{References}

1. al Naqeeb N, Edwards AD, Cowan FM, Azzopardi D. Assessment of Neonatal Encephalopathy by Amplitude-integrated Electroencephalography. Pediatrics 1999;103 (6):1263-1271

2. Anderson $\mathrm{CM}$, Torres $\mathrm{F}$, Faoro $\mathrm{A}$. The EEG of the early premature. Electroencephalogr Clin Neurophysiol 1985; 60(2): 95-105

3. Archbald F, Verma UL, Tejani NA, Handwerker SM. Cerebral function monitor in the neonate. I: Birth asphyxia. Dev Med Child Neurol 1984; 26:162-168

4. Bhushan V, Paneth N, Kiely JL. Impact of improved survival of very low birth weight infants on recent secular trends in the prevalence of cerebral palsy. Pediatrics 1993; 91(6): 1094-100

5. Biagioni E, Frisone MF, Laroche S, Rutherford M, Counsell S, Cioni G, Azzopardi D, Mercuri E, Cowan F. Occipital sawtooth: a physiological EEG pattern in very premature infants. Clin Neurophysiol 2000; 111(12): 2145-9

6. Biagioni E, Mercuri E, Rutherford M, Cowan F, Azzopardi D, Frisone MF, Cioni G, Dubowitz L. Combined use of electroencephalogram and magnetic resonance imaging in full-term neonates with acute encephalopathy. Pediatrics 2001; 107(3): 461-8

7. Bjerre I, Hellström-Westas L, Rosén I, Svenningsen NW. Monitoring of cerebral function after severe asphyxia in infancy. Arch Dis Child 1983; 58: 997-1002

8. Bolivar JM, Gerhardt T, Gonzalez A, Hummler H, Nelson C, Everett R, Bancalari E. Mechanisms for episodes of hypoxemia in preterm infants undergoing mechanical ventilation. J Pediatr 1995;127: 767-73

9. Bunt JE, Gavilanes AWD, Reulen J, Blanco CE, Vles JSH. The influence of acute hypoxemia and hypovolemic hypotension on neuronal brain activity measured by the cerebral function monitor in newborn piglets. Neuropediatrics 1996; 27: 260-264

10. Eken P, Toet MC, Groenendaal F, de Vries LS. Predictive value of early neuroimaging, pulsed Doppler and neurophysiology in full term infants with hypoxic-ischaemic encephalopathy. Arch Dis Child 1995; 73: F75-F80

11. Gavilanes AWD, Vles JSH, Sprundell R van, Siebenthal K Von, Nieman F, Reulen JPH, Blanco CE. Electrocortical brain activity, cerebral haemodynamics and oxygenation during progressive hypotension. Clin Neurophysiology 2001; 112(1): 52-59

12. Gavilanes AWD, Vles JSH, Siebenthal K Von, Sprundell R van, Reulen JPH, Blanco CE. Neonatal electrocortical brain activity and cerebral tissue oxygenation during non acidotic, normocarbic and normotensive graded hypoxemia. Submitted Clin Neurophysiology

13. Graziani LJ, Baumgart S, Desai S, Stanley C, Gringlas M, Spitzer AR. Clinical antecedents of neurologic and audiologic abnormalities in survivors of neonatal extracorporeal membrane oxygenation. J Child Neurol 1997; 12(7): 415-22 
14. Hayakawa M, Okumura A, Hayakawa F, Watanabe K, Ohshiro M, Kato Y, Takahashi R, Tauchi N. Background electroencephalographic (EEG) activities of very preterm infants born at less than 27 weeks gestation: a study on the degree of continuity. Arch Dis Child Fetal Neonatal Ed 2001; 84(3): F163-7

15. Hellström-Westas L, Rosen I, Swenningsen NW. Silent seizures in sick infants in early life. Diagnosis by continuous cerebral function monitoring. Acta Paediatr Scand 1985; 74(5): 741-8

16. Hellström-Westas L, Rosén I, Svenningsen NW. Cerebral function monitoring during the first week of life in extremely small low birthweight (ESLBW) infants. Neuropediatrics 1991; 22: 27-32

17. Hellström-Westas L, Bell AH, Skov L, Greisen G, Svenningsen NW. Cerebroelectrical depression following surfactant treatment in preterm neonates. Pediatrics 1992; 89: 643-647

18. Hellström-Westas L, Svenningsen NW, Westgren U, Rosen I, Lagerstrom PO. Lidocaine for treatment of severe seizures in newborn infants. II. Blood concentrations of lidocaine and metabolites during intravenous infusion. Acta Paediatr 1992; 81(1): 35-9

19. Hoeven MAHBM van der, Maertzdorf WJ, Blanco CE. Mixed venous oxygen saturation and biochemical parameters of hypoxia during progressive hypoxemia in 10-to 14-day-old piglets. Pediatr Res 1997; 42(6): 878-84

20. Holmes GL, Lombroso CT. Prognostic value of background patterns in the neonatal EEG.J Clin Neurophysiol 1993;10(3): 323-52

21. Keunen H, Vles JSH, Reempst JLH van, Gavilanes AWD, Hasaart THM. The effect of repetitive umbilical cord occlusions on neuronal brain activity measured by the Cerebral function Analysing Monitor and histological outcome in the immature fetal sheep. J Soc Gynecol Investig 2000; 7(4): 218-23

22. Lundstrøm KE and Greisen G. Changes in EEG, systemic circulation and blood gas parameters following two or six aliquots of porcine surfactant. Acta Pædiatr 1996; 85: 708-12

23. Martin RJ, Fanaroff AA. Neonatal apnea, bradycardia, or desaturation: does it matter? J Pediatr 1998; 132(5): 758-9

24. Reulen JPH, Gavilanes AWD, Mierlo D van, Blanco CE, Spaans F, Vles JSH. Computerised cerebral function analyzing monitor for the neonatal intensive care unit. Journal of Medical Engineering and Technology 1999; 23 (1): 29-37

25. Saarenmaa E, Huttunen P, Leppaluoto J, Meretoja O, Fellman V. Advantages of fentanyl over morphine in analgesia for ventilated newborn infants after birth: $A$ randomized trial. J Pediatr 1999; 134(2): 144-50

26. Selton D, Andre M, Hascoet JM. Normal EEG in very premature infants: reference criteria. Clin Neurophysiol. 2000; 111(12): 2116-24

27. Tin W, Milligan DWA, Pennefather P, Hey E. Pulse oximetry, severe retinopathy, and outcome at one year in babies of less than 28 weeks gestation. Arch Dis Child Fetal Neonatal Ed 2001; 84: F106-F110

28. Volpe J.. Intracranial hemorrhage. In: Neurology of the newborn. Volpe J. (ed) 4th ed. WB Saunders, Philadelphia, PA, 2000: 428493 
29. Wasunna A, Whitelaw AG. Pulse oximetry in preterm infants.Arch Dis Child. 1987; 62(9): 957-8

30. Watanabe K, Hayakawa F, Okumura A. Neonatal EEG: a powerful tool in the assessment of brain damage in preterm infants. Brain Dev 1999; 21(6): 361-72 
CHAPTER 9.

\section{GENERAL DISCUSSION AND SUMMARY}

9.1. General discussion

9.2. Main results

9.3. Main conclusions

9.4. Personal considerations

9.5. Implications for clinical practice and future research 


\subsection{General discussion}

Extensive EEG data, since Berger's first report in 1929, supports the evidence that electrical brain activity (EBA) reflects the maturity and integrity of the central nervous system and that EEG is a unique diagnostic tool for epilepsy. In regards to its prognostic use, neonatal background EEG maturational activity correlates with outcome. The more severe the EEG abnormality, the more impaired the outcome. However, predicting outcome based on a single EEG registration is discouraged (Holmes and Lombroso, 1993). In addition, serial neonatal EEGs alone are better predictors of neurological outcome than brain imaging or clinical neonatal evaluation (Tharp et al., 1989; Biagioni et al., 1996).

EBA monitoring in the neonatal intensive care however, is not implemented because of the classical EEG difficulties, such as the necessary expertise for its interpretation. The net result has been that the on-line continuous neonatal monitoring of physiologic variables does not include electrical brain activity. In this context, an important first step toward EBA monitoring has been the introduction of the cerebral function monitor (CFM) or amplitude integrated EEG (aEEG) based on pattern recognition. Clinical data on the assessment of EBA by CFM have been extensively reported since 1969 (Maynard et al.). The most relevant neonatal data are concerned with the neurological outcome of postasphyctic full-term infant. To the contrary, the present work focuses on the on-line preterm EBA monitoring and on experimental fetal and neonatal EBA data.

Impaired oxygenation, or the imbalance between oxygen supply and demand, may induce substantial systemic and neuronal effects and remains prevalent in neonatal intensive care. Therefore, this work has focused on animal experimentation of on-line monitoring of electrocortical brain activity in the fetal sheep and the neonatal piglet and in very low birth weight infants (VLBWI: $<1500 \mathrm{~g}$ birth weight) subjected to experimental or clinical hypoxemia, systemic hypotension or a combination of the two. Based on biochemical (Johnston, 2001) and hemodynamic data (Johnston et al., 2001), we hypothesized that isolated or superimposed systemic hypotension should strongly impair cerebral perfusion and subsequently EBA when compared with hypoxemia alone. Furthermore, we expected a significant increase in the slow delta $(\delta)$ EEG spectral power during hypoxemia and/or hypotension as an early marker of declined electrical brain function.

We further aimed to develop a quantitative aEEG monitor, which extracts parameters of three-EEG raw signals, to simplify, quantify and analyze EBA. The developed system is further called the Maastricht cerebral monitor (MCM, chapter 3).

\subsection{Main results}

The Maastricht Cerebral Monitor provides advanced signal analysis in the amplitude and frequency domains. It was developed with a software system called Poly (Inspektor Research System, Amsterdam, NL) for acquisition, high quality real-time display and on-/off-line EEG analysis. 
Severe hypoxemia and/or hypotension were induced in the fetal sheep and newborn piglet in order to study neuronal thresholds of dysfunction and to test the MCM. MCM data collected from fetal and neonatal animal experimentation illustrated several findings:

First, repetitive umbilical cord occlusions for 2 out of 5 minutes in the fetal sheep ( $60 \%$ of full-term gestation; chapter 4 ) showed that aEEG amplitude but not EEG spectral power analysis had already decreased during the first normotensive cord occlusion. In other words, aEEG amplitude decreased before the onset of significant hypotension, which follows at the end of the occlusion period. Further, brain histological examination showed no damage.

Secondly, acute moderate or severe hypoxemia $\left(\mathrm{SaO}_{2} 67 \pm 13.4 \%\right.$ and $28.4 \pm$ $9.3 \%$, respectively) caused no change in aEEG amplitude in newborn piglets (chapter 5). Further, no differences in aEEG or haemodynamic parameters were observed as compared to baseline registration during isolated moderate hypotension (MAP $59.3 \pm 7.8 \mathrm{mmHg}$ ). However, aEEG amplitude decreased significantly during severe systemic hypotension (MAP $35.1 \pm 13.5 \mathrm{mmHg}$ ) and during combined moderate hypotension with hypoxemia $\left(\mathrm{SaO}_{2}<85 \%\right.$ and MAP $<75 \mathrm{mmHg}$ ).

Further, we studied cerebral hemodynamics and oxygenation in the one-week old piglet. The experimental protocol used here combined for the first time NIRS and aEEG in the study of nonacidotic, normocarbic, normoxemic systemic hypotension (chapter 6) and normotensive graded hypoxemia (chapter 7) in order to correlate intracellular oxygenation and cerebral hemodynamics with electrocortical function.

During normoxemic hemorrhagic hypotension (chapter 6), MCM amplitude remained stable until the MAP, carotid blood flow $\left(Q_{\text {car }}\right)$ and oxy- + deoxyhaemoglobin $(\mathrm{tHb})$ dropped below $30 \mathrm{mmHg}(41 \%$ of baseline), 20 $\mathrm{ml} / \mathrm{min}$ (33\% of baseline) and $82 \%$ of baseline respectively. $\delta$ wave frequency percentage increased throughout the experiment and significantly at MAP below $30 \mathrm{mmHg}$. aEEG amplitude remained depressed after blood reinfusion and haemodynamic recovery. Cytaa3 changes were not statistically significant, reflecting sufficient neuronal oxygenation.

During two episodes of normotensive graded hypoxemia (chapter 7), $\delta$ EEG power increased significantly and the aEEG amplitude dropped below 10 and 5 $\mu v$ olts at the end of the first and the second hypoxemic period $\left[\mathrm{P}_{\mathrm{a}} \mathrm{O}_{2} 2.68 \pm 1,08\right.$ $(p<0.05)$ and $2.87 \pm 0.58 \mathrm{kPa}$, respectively]. Both aEEG variables normalized during recovery $\left(\mathrm{FiO}_{2}\right.$ 0.21). $\mathrm{Q}_{\text {car }}, \mathrm{CBV}$ and Cytaa3 were not changed. $\mathrm{A}$ decreasing MAP however, was seen throughout the experiment (episode a: MAP baseline $83.5 \pm 17.6 \mathrm{mmHg}$ decreased to $58.7 \pm 17.0 \mathrm{mmHg}$ at $\mathrm{FiO}_{2} 0.05$; episode b: MAP baseline of $74.5 \pm 4.3 \mathrm{mmHg}$ decreased to $47.8 \pm 13.8 \mathrm{mmHg}$ at $\mathrm{FiO}_{2}$ 0.05). The following data supports the notion that this level of systemic blood pressure throughout graded hypoxemia assured an appropriate cerebral perfusion: a) $Q_{c a r}$ remained unaltered over the hypoxemic phases. b) As shown in chapter 6 , aEEG became impaired when both MAP and $Q_{\text {car }}$ declined below $18.2 \pm 7.8 \mathrm{mmHg}$ and $9.7 \pm 3.7 \mathrm{ml} / \mathrm{min}$, respectively. c) Other authors have shown an impaired neuronal oxygenation to occur when MAP dropped below $17 \mathrm{mmHg}$ in the hypoxemic neonatal piglet (Tsuji et al., 1995). 
Finally, based on both the above described experimental data and on the expertise using the MCM, the later was introduced in the clinical setting. The impact of spontaneous normotensive hypoxemia $\left(\mathrm{SaO}_{2} 0.85\right.$ to 0.67$)$ was studied using on-line, long-term MCM in eight VLBWI (gestational age: 24 to 31 weeks; birth weight: 575-1470 g) (chapter 8). VLBWI exhibited 66 episodes of isolated hypoxemia. MCM amplitude analysis during isolated hypoxemia, relative spectral power, raw EEG and MCM amplitude patterns were comparable to baseline and recovery values.

\subsection{Main conclusions}

According to the aforementioned experimental and clinical aEEG data using the Maastricht Cerebral Monitor, we conclude that:

1. The developed Maastricht Cerebral Monitor is a flexible and user-friendly system that not only overcomes the CFM drawbacks but also provides advanced signal analysis.

2. Adaptive hemodynamic and cellular mechanisms in response to hypoxemia and/or hypotension, offer an important buffer between normal and abnormal aEEG activity.

3. aEEG alterations precede neuronal hypoxia.

4. aEEG changes during hypoxemia/hypotension in the fetal sheep differed from the observations in the newborn piglet. Reductions in aEEG amplitude occurred in response to hypoxemia earlier in the fetal sheep as compared to the one-week old piglet. In addition, the EEG power percentage revealed no changes in the fetal sheep, while shift towards the lower bands occurred during both severe graded hypoxemia and severe hypotension in newborn piglets. These data, although resulting from different animal species, may suggest the possibility that the fetal brain is more sensitive to hypoxemia than the newborn brain.

5. During severe isolated hypoxemia, neuronal (electrical) function is assured through sufficient intracellular oxygenation. Cerebral perfusion is only affected when moderate hypoxemia $\left(\mathrm{SaO}_{2}<85 \%\right)$ and moderate systemic hypotension (MAP $<75 \mathrm{mmHg}$ ) are associated in the newborn piglet.

6. The lower limit of normal cerebral perfusion in the normoxemic newborn piglet is at $30 \mathrm{mmHg}$ MAP, $20 \mathrm{ml} / \mathrm{min} \mathrm{Q}_{\mathrm{car}}$ and a tHb of $82 \%$ of baseline.

7. Extreme hypoxemia $\left(\mathrm{P}_{\mathrm{a}} \mathrm{O}_{2} 2.68 \pm 1,08 \mathrm{kPa}\right)$ is needed to induce EBA dysfunction in the normotensive newborn piglet.

8. Normotensive VLBWI maintained an unchanged electrical brain activity whilst under isolated, moderate acute hypoxemia $\left(\mathrm{SaO}_{2} 0.67\right.$ to 0.85$)$. These data suggest that the limit of hypoxemia used in clinical medicine $\left(\mathrm{SaO}_{2}\right.$ of 0.85$)$ is significant higher than the neuronal hypoxic threshold.

9. According to points 5, 6, 7 and 8: cerebral perfusion is more important than oxygen delivery for the maintenance of neuronal oxygenation.

10. The evidence with respect to the EEG $\delta$ power as an early marker for brain dysfunction is inconsistent though there is a trend toward augmentation. This can be partly explained by the fact that the animals were under anaesthesia.

11. As previously stated, aEEG does not replace conventional multi-channel 
routine EEG in the clinical setting.

\subsection{Personal considerations}

I would like to communicate some thoughts on my experiences during the production of this thesis.

I started this work, based on the fact that EEG is an excellent tool to depict brain function, under the assumption of acute neuronal changes in response to hypoxemia and/or hypotension in experimental models and in VLBWI. The observations however, clearly demonstrate that neuronal damage commences only after the failure of long-standing adaptive mechanisms. These results, far from discouraging me, enriched my knowledge and stimulated further research questions. In addition, the present work gave me the personal satisfaction to be part of a multidisciplinary team working in a field that we appreciate and enjoy.

The general aim of this thesis was directed both towards the development of a flexible aEEG system and to the study of the neonatal brain with particular attention given to the effects of impaired oxygenation. We have fulfilled this aim.

\subsection{Implications for clinical practice and future research}

From the results of the present work we think that the use of aEEG for continuous EBA monitoring has an important place in the neonatal intensive care. The Maastricht Cerebral Monitor is well suited for this purpose.

Sustained development however implicates, further work and new objectives: Our current clinical research focuses on the role of normoxemic hypotension in VLBWI and the study of early aEEG changes in the post-asphyctic VLBWI. Further, the simultaneous storage of physiologic variables and aEEG activity by the Maastricht Cerebral Monitor is pending and will enhance its research application.

Finally, the use of mathematical algorithms in the automated detection of aEEG events or abnormalities such as seizures and inappropriate background activity is under consideration by other authors. The results of their research are very encouraging (Gotman et al., 1997; Hellstrom-Westas L, 2002). 


\section{ALGEMENE DISCUSSIE EN SAMENVATTING}

9.1. General discussion

9.2. Belangrijkste resultaten

9.3. Belangrijkste conclusies

9.4. Persoonlijke overwegingen

9.5. Implicaties voor de praktijk en toekomstige onderzoeken 


\subsection{Algemene discussie}

Sinds het eerste verslag van Berger in 1929, is er een aanzienlijke hoeveelheid gegevens die het bewijs ondersteunen dat elektrische hersenactiviteit een weergave is van de ontwikkeling en de integriteit van het centraal zenuwstelsel en dat EEG een uniek diagnostisch middel is ten aanzien van epilepsie. Wat de prognose betreft, is er een correlatie tussen het neonatale achtergrondpatroon op het EEG en de outcome. Hoe ernstiger de afwijkingen op het EEG, hoe slechter de outcome. Het voorspellen van de outcome aan de hand van een enkele EEGregistratie wordt echter afgeraden (Holmes and Lombroso, 1993). Daar tegenover staat dat opeenvolgende neonatale EEG's een betere voorspellende waarde hebben dan beeldvorming van het brein of klinische evaluatie van de pasgeborene (Tharp et al., 1989; Biagioni et al., 1996).

Het monitoren van elektrische hersenactiviteit wordt echter nog niet geïmplementeerd in de neonatale intensieve care vanwege klassieke barrières zoals de expertise die vereist is voor de interpretatie ervan. Het netto resultaat hiervan is dat de elektrische hersenactiviteit geen deel uitmaakt van het continu monitoren van fysiologische variabelen bij pasgeborenen. In deze context is de introductie van de cerebral function monitor (CFM) of amplitude integrated EEG (aEEG) een belangrijke eerste stap geweest. Sinds 1969 (Maynard et al.) zijn er op grote schaal klinische gegevens gepubliceerd over meting van elektrische hersenactiviteit met behulp van CFM. De meest relevante data wat betreft pasgeborenen beschrijven de neurologische outcome van post-asfyctische à terme pasgeborenen. Aan de andere kant richt dit huidige onderzoek zich op het on-line monitoren van elektrische hersenactiviteit bij prematuren en op experimentele foetale en neonatale EBA gegevens.

Een verminderde zuurstofvoorziening dan wel een verstoring van de balans tussen de vraag naar en de aanvoer van zuurstof, kunnen ernstige systemische en neuronale effecten met zich meedragen en zijn entiteiten die aanwezig blijven in de neonatale intensive care. Om die reden richt dit werk zich op dierexperimenteel onderzoek door middel van het online monitoren van elektrische hersenactiviteit bij foetale lammeren en pasgeboren biggen en bij pasgeborenen met een zeer laag geboortegewicht (very low birthweight infants, VLBWI: geboortegewicht $<1500$ gram). Deze worden blootgesteld aan experimentele dan wel klinische hypoxemie of hypotensie dan wel een combinatie van beide. Op grond van biochemische (Johnston, 2001) en hemodynamische gegevens (Johnston et al., 2001) stelden wij de hypothese op dat geïsoleerde dan wel gesuperponeerde systemische hypotensie een groter negatief effect zou hebben op de cerebrale perfusie, en daarmee de elektrische hersenactiviteit, dan hypoxemie alleen. Daarbij verwachtten we een significante toename van de kracht van de langzame $\delta$-golven in het EEGspectrum tijdens hypoxemie en/of hypotensie als een vroeg teken van een verslechterde elektrische hersenactiviteit.

Verder hebben we ons erop gericht om een kwantitatieve aEEG te ontwikkelen die gegevens verkrijgt via ruwe signalen van een driekanalen-EEG, om op die manier elektrische hersenactiviteit te kunnen vereenvoudigen, te kwantificeren en te analyseren. Deze monitor wordt verder de Maastricht Cerebral Monitor (MCM, hoofdstuk 3) genoemd. 


\subsection{Belangrijkste resultaten}

De MCM geeft een geavanceerde signaal-analyse van de amplitude- en frequentiedomeinen. Voor het verkrijgen van de gegevens en de mogelijkheden van een hoog kwalitatieve real-time display en on/off-analyse, werd de MCM ontwikkeld met een software-systeem Poly (Inspektor Research System, Amsterdam, NL).

Om de drempelwaarde van neuronale disfunctie te bestuderen en de MCM te testen, werden foetale lammeren en pasgeboren biggen blootgesteld aan ernstige hypoxemie en/of hypotensie. Gegevens verkregen met de MCM door middel van foetaal en neonataal dierexperimenteel onderzoek leidden tot verscheidene bevindingen:

Ten eerste bleek dat bij herhaaldelijk afsluiten van de navelstreng bij foetale lammeren (60\% van voldragen leeftijd, hoofdstuk 4) gedurende 2 per 5 minuten, reeds bij de eerste normotensieve periode van afsluiting de amplitude op het aEEG een afname toonde, terwijl de EEG spectrale power-analyse onveranderd bleef. Daarbij was histologisch onderzoek van de hersenen zonder afwijkingen.

Een tweede bevinding was, dat er geen afwijkingen te zien waren in de amplitude van het aEEG bij pasgeboren biggen tijdens acute matige en ernstige hypoxemie $\left(\mathrm{SaO}_{2}\right.$ respectievelijk $67 \pm 13,4 \%$ en $28,4 \pm 9,3 \%$ ) (hoofdstuk 5). Daarbij werden er tijdens geïsoleerde matige hypotensie (MAP 59,3 $\pm 7,8 \mathrm{mmHg}$ ) geen veranderingen gezien van de aEEG en hemodynamische parameters ten opzichte van de baseline-registratie. Echter, tijdens ernstige systemische hypotensie (MAP 35,1 \pm $13,5 \mathrm{mmHg}$ ) en gecombineerde matige hypotensie en hypoxemie (MAP $<75$ $\mathrm{mmHg}$ en $\mathrm{SaO}_{2}<85 \%$ ) was er een significante afname van de amplitude op het aEEG.

In een later stadium bestudeerden we de cerebrale hemodynamiek en zuurstofvoorziening bij biggen van een week oud. Ons experimentele protocol combineerde voor het eerst NIRS en aEEG bij het bestuderen van niet-acidotische, normocapnische en normoxemische systemische hypotensie (hoofdstuk 6) en normotensieve gegradeerde hypoxemie (hoofdstuk 7). Op deze manier werden intracellulaire oxygenatie en cerebrale hemodynamiek gecorreleerd aan electrocorticale functie.

Tijdens normoxemische hemorrhagische hypotensie bleef de amplitude op de MCM stabiel totdat de MAP daalde tot onder de $30 \mathrm{mmHg}(41 \%$ van de baselinewaarde), de $Q_{\text {car }}$ tot onder de $20 \mathrm{ml} / \mathrm{min}$ (33\% van de baseline-waarde) en de $\mathrm{tHb}$ tot onder de $82 \%$ van de baseline-waarde. Tijdens het experiment nam het percentage $\delta$-golf-frequentie toe en deze toename werd significant bij een MAP onder de $30 \mathrm{mmHg}$.De amplitude op het bleef verlaagd na bloed-reinfusie en hemodynamisch herstel. Veranderingen van het Cytaa3 waren niet statistisch significant, wat weergeeft dat de neuronale oxygenatie toereikend bleef.

Tijdens twee periodes van normotensieve, gegradeerde hypoxemie (hoofdstuk 7 ) nam de $\delta$-kracht op het EEG significant toe en de amplitude van het aEEG af tot respectievelijk onder de 10 en $5 \mu$ volt aan het eind van de twee periodes van hypoxemie ( $\mathrm{PaO} 2$ respectievelijk 2,68 $\pm 1,08(\mathrm{p}<0,05)$ en 2,87 $\pm 0,58 \mathrm{kPa})$. Deze beide EEG-variabelen normaliseerden gedurende de herstelfase (FiO2 21\%). De 
$Q_{\text {car }}$, het CBV en het Cytaa3 bleven onveranderd. Wel werd een dalende MAP gezien gedurende het experiment (episode a: baseline-MAP $83 \pm 17,6 \mathrm{mmHg}$, dalend tot $58,7 \pm 17,0 \mathrm{mmHg}$ bij een FiO2 van $5 \%$; episode b: baseline-MAP 74,5 \pm $4,3 \mathrm{mmHg}$, dalend tot $47,8 \pm 13,8 \mathrm{mmHg}$ bij een $\mathrm{FiO} 2$ van $5 \%$ ). De nu volgende gegevens ondersteunen echter de vaststelling dat door de systemische bloeddruk een afdoende cerebrale bloedtoevoer verzekerd werd gedurende dit experiment van gegradeerde hypoxemie: a) de $Q_{\text {car }}$ bleef onveranderd tijdens de hypoxemische periodes; b) zoals we kunnen zien in hoofdstuk 6 , verslechterde het aEEG pas op het moment dat de MAP daalde tot onder de $18,2 \pm 7,8 \mathrm{mmHg}$ en de $Q_{\text {car }}$ tot onder de 9,7 $\pm 3,7 \mathrm{ml} / \mathrm{min}$; en c) eerdere experimenten bij hypoxemische pasgeboren biggen lieten pas een verslechterde neuronale oxygenatie zien bij een daling van de MAP onder de $17 \mathrm{mmHg}$ (Tsuji et al., 1995).

Tenslotte, op basis van de hierboven beschreven experimentele gegevens over het gebruik van de MCM, werd deze in de klinische setting geïntroduceerd. Bij acht VLBWI (geboren bij zwangerschapstermijn 24-31 weken; geboortegewicht 5751470 gram) werd de invloed van spontane, normotensieve hypoxemie $\left(\mathrm{SaO}_{2} 67-\right.$ $85 \%)$ geëvalueerd met behulp van langdurige on-line monitoring met de MCM (hoofdstuk 8). Deze VLBWI hadden totaal 66 episodes van geïsoleerde hypoxemie. De uitkomsten van amplitude-analyse van de MCM, de relatieve spectrale kracht, het ruwe EEG en de amplitudepatronen op de MCM tijdens de geïsoleerde hypoxemie waren vergelijkbaar met de baseline-waarden en de waarden tijdens de herstelfase.

\subsection{Belangrijkste conclusies}

Naar aanleiding van de hierboven besproken experimentele en klinische aEEGgegevens verkregen via de MCM, kunnen we de volgende conclusies trekken:

1. De ontwikkelde MCM is een flexibel en gebruiksvriendelijk systeem dat niet alleen de nadelen van de CFM overwint, maar ook een geavanceerde signaalanalyse mogelijk maakt.

2. Hemodynamische en cellulaire aanpassingsmechanismen als reactie op hypoxemie en/of hypotensie vormen een belangrijke buffer tussen normale en afwijkende activiteit op het aEEG.

3. Veranderingen in het aEEG gaan vooraf aan neuronale hypoxie.

4. De veranderingen in het $\mathrm{aEEG}$ verschillen tussen foetale lammeren en pasgeboren biggen. In vergelijking met pasgeboren biggen treedt er bij foetale lammeren eerder een afname van de amplitude op het aEEG op. Daarbij traden er geen veranderingen in het spectrale power van het EEG op bij de foetale lammeren, terwijl er bij de pasgeboren biggen een verschuiving optrad richting de lagere banden tijdens zowel ernstige gegradeerde hypoxemie als ernstige hypotensie. Hoewel deze gegevens verkregen zijn bij verschillende diersoorten, suggereren ze dat het foetale brein gevoeliger is voor hypoxemie dan het brein van de pasgeborene.

5. Tijdens ernstige geïsoleerde hypoxemie wordt de neuronale (elektrische) functie verzekerd door een afdoende intracellulaire oxygenatie. Bij pasgeboren biggen is de cerebrale perfusie aangedaan wanneer matige systemische hypotensie (MAP < $75 \mathrm{mmHg}$ ) samengaat met matige hypoxemie $\left(\mathrm{SaO}_{2}<85 \%\right)$.

6 . De ondergrens van een normale cerebrale perfusie bij de normoxemische 
pasgeboren biggen is veilig gesteld bij een MAP van $30 \mathrm{mmHg}$, een $\mathrm{Q}_{\text {car }}$ van 20 $\mathrm{ml} / \mathrm{min}$ en een $\mathrm{tHb}$ op $82 \%$ van de baseline-waarde.

7. $\mathrm{Er}$ is een extreme hypoxemie ( $\mathrm{PaO} 22,86 \pm 1,08 \mathrm{kPa})$ nodig om bij pasgeboren biggen afwijkingen op het aEEG te bewerkstelligen.

8. Normotensieve VLBWI toonden een onveranderde elektrische hersenactiviteit gedurende acute, matige, geïsoleerde hypoxemie $\left(\mathrm{SaO}_{2} 67-85 \%\right)$. Dit suggereert dat de grens van hypoxemie zoals die wordt gehanteerd in de klinische setting $\left(\mathrm{SaO}_{2} 85 \%\right)$ significant hoger is dan de drempelwaarde voor neuronale hypoxie.

9. Uit de punten $5 \mathrm{t} / \mathrm{m} 8$ kan worden afgeleid dat de mate van cerebrale perfusie belangrijker is voor het behoud van een goede intraneuronale oxygenatie dan de zuurstof content.

10. Aanwijzingen voor de bruikbaarheid van EEG $\delta$-power als een vroege marker voor disfunctie van het brein zijn niet consistent, hoewel er een neiging is tot een toename ervan. Dit gebrek aan consistentie kan deels verklaard worden door het feit dat de dieren onder anesthesie waren.

11. Zoals reeds eerder gesteld kan het aEEG geen vervanging zijn van het routine multichannel-EEG in de klinische setting.

\subsection{Persoonlijke overwegingen}

Graag zou ik enkele gedachten uitwerken met betrekking tot de ervaringen zoals ik die heb opgedaan tijdens het proces van dit proefschrift.

Ik ben dit werk begonnen gebaseerd op het feit dat het EEG een uitstekend instrument is om hersenactiviteit in beeld te brengen en met de aanname dat er acute neuronale veranderingen zouden optreden als reactie op hypoxemie en/of hypotensie in experimentele modellen en bij VLBWI. De bevindingen van dit onderzoek tonen echter duidelijk dat neuronale schade pas optreedt na falen van langdurige aanpassingsmechanismen. In tegenstelling tot dat deze resultaten mij ontmoedigden, waren ze een verrijking voor mijn kennis en stimuleerden me tot het ontwikkelen van verdere onderzoeksvragen. Daarbij gaf het huidige werk me de persoonlijke voldoening om deel uit te maken van een multidisciplinair team dat werkt in een onderzoeksveld dat ze waardeert en waarvan ze geniet.

Het algemene doel van dit proefschrift was zowel het ontwikkelen van een flexibel aEEG-systeem als het bestuderen van het neonatale brein, met speciale aandacht voor de effecten van een verminderde oxygenatie hierop. Dit doel hebben we bereikt.

\subsection{Implicaties voor de praktijk en toekomstige onderzoeken}

Vanuit de resultaten van ons huidige onderzoek denken we dat het gebruik van het $\mathrm{aEEG}$ als een continue monitoring van elektrische hersenactiviteit een plek heeft in de huidige neonatale intensive care. De MCM is zeer geschikt voor dit doel.

Een aanhoudende ontwikkeling impliceert echter aanhoudend onderzoek en nieuwe doelen: ons huidige onderzoek richt zich op de rol van normoxemische hypotensie in VLBWI en op het bestuderen van vroege veranderingen op het aEEG bij post-asfyctische VLBWI. Daarnaast wordt er gewerkt aan een gelijktijdige opslag van fysiologische variabelen en aEEG-activiteit met de MCM, iets dat ook de mogelijkheden voor toepassing ten behoeve van onderzoek zal vergroten. 
Tenslotte wordt er door verscheidene auteurs nagedacht over het gebruik van wiskundige algoritmes voor een geautomatiseerde detectie van gebeurtenissen op het aEEG en afwijkingen zoals epileptische aanvallen en een afwijkend achtergrondpatroon. De resultaten van die onderzoeken zijn zeer aanmoedigend (Gotman et al., 1997; Hellstrom-Westas L, 2002). 


\section{DISCUSION GENERAL Y RESUMEN}

9.1. Discusión general

9.2. Resultados principales

9.3. Conclusiones principales

9.4. Implicaciones prácticas e investigación futura 


\subsection{Discusión general}

Desde el primer reporte de Berger en 1929, un volumen importante de datos apoyan el hecho que la actividad eléctrica cerebral (AEC) refleja la madurez e integridad del sistema nervioso central y que el EEG es un medio de diagnóstico único en la epilepsia. En lo que respecta al valor pronóstico del EEG, existe una correlación entre la actividad de base del EEG neonatal con las consecuencias clínicas a largo plazo. Mientras más severa la anomalía del EEG más importantes serán estas consecuencias. Sin embargo, predicciones clínicas en base a un solo EEG son poco recomendables (Holmes and Lombroso, 1993). Adicionalmente, EEG's neonatales sucesivos podrían tener un mayor valor predictivo de patología neurológica que el diagnóstico de imagen cerebral o que la evaluación clínica neonatal (Tharp et al., 1989; Biagioni et al., 1996).

A pesar de su importancia, el monitoreo de la actividad eléctrica cerebral no está implementado en los cuidados intensivos neonatales debido a las clásicas dificultades de interpretación del EEG. El resultado neto es que el monitoreo continuo de variables fisiológicas del neonato no incluyen a la actividad eléctrica cerebral. En este contexto, la introducción del monitor de función cerebral (MFC) o EEG de amplitud integrada (aEEG) basado en reconocimientos de patrones ha constituido el primer paso hacia el monitoreo de la AEC neonatal. Información clínica basada en el monitoreo del AEC por medio del MFC ha sido extensivamente reportada desde 1969 (Maynard et al.). La información neonatal más relevante concierne a las consecuencias neurológicas de recién nacidos post-asfícticos a término. Al contrario, este trabajo se centra en el monitoreo en línea de la AEC del paciente pretérmino y en la investigación de la AEC experimental fetal y neonatal.

Trastornos de oxigenación, o el inbalance entre abastecimiento y demanda de oxígeno, son entidades que prevalecen en los cuidados intensivos neonatales y que pueden inducir importantes efectos sistémicos y neuronales. Por este motivo el presente trabajo se ha enfocado en la experimentación animal del monitoreo en línea de la AEC del feto del cordero y de cerdos recién nacidos, así como de recién nacidos con muy bajo peso al nacer (very low birthweight infants, VLBWI: peso al nacer < $1500 \mathrm{gr}$ ). Estos han sido sujetos a hipoxemia experimental o clínica, hipotensión sistémica o a ambas. Basándonos en información bioquímica (Johnston, 2001) y hemodinámica (Johnston, et al., 2001), nos planteamos la hipótesis que la hipotensión sistémica aislada o la superimpuesta a la hipoxia debería tener un efecto negativo mas importante en la perfusión cerebral y por consiguiente en la $A E C$, que la hipoxia aislada. Además, esperabamos un incremento importante de la frecuencia delta $(\delta)$ del EEG durante la hypoxemia y/o hipotensión, como un marcador temprano de deterioro de la actividad eléctrica cerebral. Adicionalmente, nos propusimos desarrollar un monitor aEEG cuantitativo que extrayera parámetros de 3 señales crudas de EEG, para simplificar, cuantificar y analizar la AEC. El sistema desarrollado ser nombrado en lo sucesivo Monitor Cerebral Maastricht (MCM, capítulo 3). 


\subsection{Resultados principales}

MCM provee un análisis avanzado en los dominios de amplitud y frecuencia del EEG. Se desarrollo utilizando el sistema programa de computación Poly (Inspektor Research System, Amsterdam, NL), para la adquisición, proyección en tiempo real y análisis de EEG en-o fuera de línea.

Hipoxemia severa y/o hipotensión fueron inducidas en el feto de cordero y en el cerdo recién nacido, con el fin de estudiar los límites de disfunción neuronal y además para probar el sistema MCM. Los datos de investigación arrojan los siguientes resultados:

1. Oclusiones repetitivas del cordón umbilical durante 2 de cada 5 minutos en el feto del cordero (60\% de gestación completa; capítulo 4), deprimen immediatamente la amplitud del aEEG, sin afectar jamás las bandas de frecuencias del EEG. Además, el examen histológico cerebral no presentó daño alguno.

2. Periodos agudos de hipoxia moderada o severa $\left(\mathrm{SaO}_{2} 67 \pm 13.4 \%\right.$ and $28.4 \pm$ 9.3, respectivamente) no afectan la amplitud del aEEG en cerdos recién nacidos (capitulo 5). Además, no se presentaron cambios hemodinámicos ni de aEEG durante la hipotensión moderada (MAP $59.3 \pm 7.8 \mathrm{mmHg}$ ). Sin embargo, la amplitud del aEEG decreció significativamente durante la hipotensión sistémica severa (MAP $35.1 \pm 13.5 \mathrm{mmHg}$ ) y durante la combinación de hipotensión moderada e hipoxemia $\left(\mathrm{SaO}_{2}<85 \%\right.$ y $\left.\mathrm{MAP}<75 \mathrm{mmHg}\right)$.

3. La hipotensión hemorrágica durante normoxemia (capitulo 6) induce una depresión de la amplitud del aEEG cuando la presión media arterial (MAP), flujo sanguineo carotideo $\left(Q_{\mathrm{car}}\right)$ y la oxy- + deoxyhemoglobina $(\mathrm{tHb})$ dsminuyeron a niveles inferiores a $30 \mathrm{mmHg}(41 \%$ de linea de base), $20 \mathrm{ml} / \mathrm{min}$ (33\% de la línea de base) y $82 \%$ de la línea de base, respectivamente. El porcentaje de la frequencia $\delta$ se incremento a lo largo de todo el experimento, pero adquirió significancia cuando la MAP se situó por debajo de $30 \mathrm{mmHg}$. La amplitud del aEEG se mantuvo deprimida a pesar de la reinfusión de sangre y de la recuperación hemodinámica. Cambios del Cytaa3 no fueron estadísticamente significativos, reflejando una oxigenación neuronal adequada.

4. Dos periodos de hipoxemia gradual durante normotensión (chapter 7) demostraron un incremento de la banda $\delta$ del EEG y la caída de la amplitud del aEEG por debajo de los 10 y $5 \mu$ volts al final de ambos periodos hipoxémicos $\left[\mathrm{P}_{\mathrm{a}} \mathrm{O}_{2} 2.68 \pm 1,08(\mathrm{p}<0.05)\right.$ y $2.87 \pm 0.58 \mathrm{kPa}$, respectivamente]. Ambas variables del EEG se normalizaron durante el periodo de recuperación $\left(\mathrm{FiO}_{2} 0.21\right) . \mathrm{Q}_{\mathrm{car}}$, CBV y Cytaa3 no presentaron cambios. La MAP tendió a bajar durante todo el experimento sin alcanzar importancia estadística (episodio a: MAP línea de base $83.5 \pm 17.6 \mathrm{mmHg}$ decreció a $58.7 \pm 17.0 \mathrm{mmHg}$ con una $\mathrm{FiO}_{2}$ 0.05; episodio b: MAP línea de base $74.5 \pm 4.3 \mathrm{mmHg}$ decrecióó a $47.8 \pm 13.8 \mathrm{mmHg}$ con una $\mathrm{FiO}_{2}$ 0.05). A pesar de esto, los siguientes datos apoyan la noción que la presión sanguinea sistémica aseguró una adecuada perfusión cerebral en este ultimo experimento: a) $Q_{\text {car }}$ fue constante durante las dos fases hypoxémicas. b) En el experimento descrito en el capítulo 6 demostramos que 
la amplitud del aEEG es afectada cuando la MAP y el $Q_{\text {car }}$ se situan por debajo de $18.2 \pm 7.8 \mathrm{mmHg}$ y $9.7 \pm 3.7 \mathrm{ml} / \mathrm{min}$, respectivamente. c) Otros autores han demostrado que la oxigenación neuronal se afecta en el cerdo recien nacido cuando la MAP disminuye por debajo de los $17 \mathrm{mmHg}$ (Tsuji et al., 1995).

5. Finalmente, el MCM fue aplicado en el estudio de la actividad eléctrica cerebral del recién nacido de muy bajo peso al nacer $(<1500 \mathrm{gr})$ sujeto a hypoxemia normotensiva aguda moderada $\left(\mathrm{SaO}_{2} 0.85\right.$ to 0.67$)$. Se registraron 8 prematuros (edad gestacional: 24 a 31 semanas; peso al nacer: $575-1470 \mathrm{~g}$ ) con MCM en linea y a largo plazo (chapter 8). aEEG y EEG no manifestaron cambios.

\subsection{Conclusiones principales}

1. El MCM es un sistema flexible y de uso fácil que no solamente supera la dificultades de uso del CFM, sino que también incluye análisis avanzados de la señal de EEG.

2. Los mecanismos hemodinámicos y celulares en respuesta a la hipoxemia y/o hipotensión, aseguran un margen de adaptación importante.

3. Alteraciones del aEEG preceden a la hipoxia neuronal.

4. Cambios del aEEG durante hipoxemia/hipotensión en el feto del cordero difieren de las observaciones hechas en el cerdo recién nacido. La reducción de la amplitud del aEEG en respuesta a la hipoxemia es inmediata en el feto del cordero. Ademas, el porcentaje de frecuencia del EEG se mantiene inalterado en el feto del cordero, mientras que frecuencias mas lentas son reproducidas en el cerdo recien nacido durante hipoxemia gradual grave e hipotensión severa. Estos datos, aunque de dos especies de animales diferentes, sugieren la posibilidad de que el cerebro fetal sea mas sensible a la hipoxemia que el neonatal.

5. La función eléctrica neuronal esta asegurada por una oxigenación intracelular adecuada durante la hipoxemia aislada en el cerdo recién nacido. La perfusión cerebral se afecta únicamente cuando la hipoxemia moderada $\left(\mathrm{SaO}_{2}<85 \%\right)$ esta asociada con hipotensión sistémica moderada (MAP < $75 \mathrm{mmHg}$ ).

6. El límite de la normalidad de la perfusión cerebral en el cerdo recién nacido normoxémico se ubica a una MAP de $30 \mathrm{mmHg}, \mathrm{Q}_{\text {car }}$ de $20 \mathrm{ml} / \mathrm{min}$ y $\mathrm{tHb}$ op $82 \%$.

7. Se necesita una hipoxemia extrema $\left(\mathrm{PaO}_{2} 2,86 \pm 1,08\right)$ para inducir disfunción electrica cerebral en el cerdo recien nacido normotensivo.

8. Prematuros de muy bajo peso al nacer mantienen una actividad eléctrica cerebral inalterada durante la hipoxemia moderada aislada $\left(\mathrm{SaO}_{2} 67-85 \%\right)$. Esto sugeriría que el límite de saturación de oxígeno de $86 \%$ aceptado en la clínica es significativamente mas alto que aquel que corresponde al umbral de la hipoxia neuronal.

9. De acuerdo a los puntos 5,6,7 y 8 : la perfusión cerebral es mas importante que el contenido de oxígeno sanguíneo para el mantenimiento de la función neuronal.

10. La evidencia con respecto a la frecuencia delta del EEG como un marcador temprano de disfunción cerebral es inconsistente en nuestros ecxperimentos. 
El hecho de que los animales estaban bajo la influencia de anestésicos, podría explicar en parte este hecho.

11. El aEEG no reemplaza al EEG convencional.

\subsection{Implicaciones para la práctica clínica y líneas de investigación}

Al presente, nuestro trabajo de investigación clínica se centra en el rol de la hipotensión aislada en la actividad elèctrica cerebral de prematuros con muy bajo peso al nacer y en los cambios tempranos en el aEEG inducidos por la asfixia del recién nacido pretèrmino. Adicionalmente, esperamos incluir e a corto plazo el registro simultaneo de variabes fisiológicas junto con el del aEEG. Esto mejorará la aplicación del MCM en la clínica y en el laboratorio.

Finalmente cabe anotar que el uso de algorrítmos matemáticos de detección automática de eventos, tales como convulsiones o ritmos de base abnormales, están siendo considerados por otros autores (Gotman et al., 1997; HellstromWestas L, 2002). 


\section{References}

Biagioni E, Bartalena L, Biver P, Pieri R, Cioni G. Electroencephalographic dysmaturity in preterm infants: a prognostic tool in the early postnatal period. Neuropediatrics. 1996; 27(6): 311-6.

Gotman J, Flanagan D, Rosenblatt B, Bye A, Mizrahi EM. Evaluation of an automatic seizure detection method for the newborn EEG. Electroencephalogr Clin Neurophysiol. 1997; 103(3): 363-9.

Hellstrom-Westas $L$. Cerebral function monitoring. In: The newborn brain: neuroscience and clinical applications. Ed. Lagercrantz $\mathrm{H}$ et al. Cambridge University Press 2002, Cambridge - UK

Holmes GL, Lombroso CT. Prognostic value of background patterns in the neonatal EEG. J Clin Neurophysiol. 1993; 10(3): 323-52.

Johnston MV. Excitotoxicity in neonatal hypoxia. Ment Retard Dev Disabil Res Rev. 2001; 7(4): 229-34.

Johnston MV, Trescher WH, Ishida A, Nakajima W. Neurobiology of hypoxicischemic injury in the developing brain. Pediatr.Res. 2001; 49(6): 735-41.

Maynard D, Prior PF, Scott DF. A continuous monitoring device for cerebral activity. Electroencephalogr Clin Neurophysiol. 1969a; 27(7): 672-3.

Tharp BR, Scher MS, Clancy RR. Serial EEGs in normal and abnormal infants with birth weights less than 1200 grams--a prospective study with long term follow-up. Neuropediatrics. 1989; 20(2): 64-72.

Tsuji M, Naruse H, Volpe J, Holtzman D. Reduction of Cytochrome aa3 measured by Near-Infrared Spectroscopy Predicts Cerebral Energy Loss in Hypoxic Piglets. Pediatr Res 1995;37:253-259.

Wauquier A. EEG and neuropharmacology. In: Niedermeyer E. and Lopesda Silva F. Electroencephalography. Basic principles, clinical applications, and related fields. 3th ed. Williams \& Wilkins, 1993: 619-629. 


\section{ACKNOWLEDGMENTS}

Throughout the years many people in Ecuador, Belgium and The Netherlands have contributed to my medical career and to this PhD project, I wish to express my gratitude to all of them. Most of all, I thank Professor Carlos Blanco and Professor Hans Vles, promotors of this thesis. I appreciate their thoughtful ideas and critical comments on the research designs and conclusions drawn from this thesis, as well as their important contribution to my clinical work. Furthermore Professor Vles, has shown me the importance of cultivating my family's bonds, for this my gratitude. With respect I thank my co-promotor Dr. Jos Reulen, who was always available for discussion and comments. I also wish to express my thanks to Dr. Kurt von Siebenthal for his suggestions and critical remarks, and to Dr. Vivianne van Kranen-Mastenbroek for her feedback and interest. Equally, I thank Dr. Jan Eric Bunt and Dr. Han Keunen for their outstanding participation in this project.

Further, I thank my colleages and friends Wiel Maertzdorf, Pieter Degrauwe, Mark van der Hoeven, Twan Mulder and Eduardo Villamor for the close collaboration in our daily work. Finally, I thank the nursing staff of the NICU for its interest and assistance.

This thesis is dedicated to my dear ones.

Esta tesis está dedicada a mis seres queridos. 


\section{CURRICULUM VITAE}

A.W. Danilo Gavilanes Jimenez

Born 29th of April 1960 in Guayaquil - Ecuador

Department of Pediatrics, Division of Neonatology

University Hospital Maastricht

P. Debijelaan 25, 6202 AZ Maastricht

The Netherlands

Tel: +31-43-3877246

Fax:+31-43-3875246

E-mail: aga@paed.azm.nl

\section{EDUCATION}

1972-1978 Secondary German School, Guayaquil - Ecuador

1978-1985 Faculty of Medicine, Catholic University of Guayaquil,

Guayaquil - Ecuador

1986-1991 Pediatric Residency Program, Catholic University of Leuven, Leuven - Belgium

1989 Course of Tropical Medicine, Institute of Tropical Medicine "Prince Leopold", Antwerp - Belgium

1993-1995 Neonatal Fellowship Program, University Hospital Maastricht, Maastricht - The Netherlands

\section{ACADEMIC DISTINCTIONS}

1982-1984 Undergraduate Lecturer on Embryology,

Catholic University of Guayaquil - Ecuador

1986-1992 International Scholarship for Pediatric Residency Program, Catholic University of Leuven, Leuven - Belgium

\section{LICENSES}

1985

Medical Doctor

1990

Diploma of Tropical Medicine

1991

Pediatrician

1995

Neonatologist

\section{PROFESSIONAL}

1985-1986 General Practitioner, Ministry of Health, Guayaquil - Ecuador 1991-1993 Pediatrician, General Hospital St. Jan, Genk - Belgium

Since 1995 Neonatologist, Univ. Hospital Maastricht, Maastricht - The Netherlands 


\section{PUBLICATIONS BELONGING TO THIS THESIS}

JE Bunt, AWD Gavilanes, J Reulen, CE Blanco, JSH Vles. The influence of acute hypoxemia and hypovolemic hypotension on neuronal brain activity measured by the cerebral function monitor in newborn piglets. Neuropediatrics 1996; 27:260-264

JPH Reulen, AWD Gavilanes, D van Mierlo, CE Blanco, F Spaans, JSH Vles. Computerised cerebral function analyzing monitor for the neonatal intensive care unit. Journal of Medical Engineering and Technology 1999 23:1; 29-37

H Keunen, JSH Vles, JLH van Reempst, AWD Gavilanes, THM Hasaart. The effect of repetitive umbilical cord occlusions on neuronal brain activity measured by the Cerebral function Analysing Monitor and histological outcome in the immature fetal sheep. J Soc Gynecol Investig $2000 ; 7(4): 218-23$

AWD Gavilanes, JSH Vles, R Van Sprundell, K Von Siebenthal, F Nieman, JPH Reulen, CE Blanco. Electrocortical brain activity, cerebral haemodynamics and oxygenation during progressive hypotension. Clin Neurophysiology 2001;112(1):5259

AWD Gavilanes, JSH Vles, K Von Siebenthal, R Van Sprundell, JPH Reulen, CE Blanco. Neonatal electrocortical brain activity and cerebral tissue oxygenation during non acidotic, normocarbic and normotensive graded hypoxemia.

Clin Neurophysiology, in press

AWD Gavilanes, JSH Vles, VHJM van Kranen-Mastenbroek, JPH Reulen, M.van Reisen and CE Blanco. Electrocortical brain activity during normotensive hypoxemia in VLBWI undergoing intensive care.

Neuropediatrics, accepted

\section{PUBLICATIONS}

R. Mena and AWD Gavilanes. Notebook of Basic Data and Practical Annotations of Embryology . A consultation guide of practical Embryology. Faculty of Medicine, 1983 Catholic University of Guayaquil, Guayaquil - Ecuador.

I. Surmont, AWD Gavilanes, J Van de Pitte, H Devlieger, E Eggermont. Malassezia Furfur fungaemia in infants receiving intravenous lipid emulsions. A rarity of just underestimated? European Journal of Pediatrics 1989; 148:435-438

P Brienesse, L Anteunis, H Wit, AWD Gavilanes, W Maertzdorf. Otoacoustic emissions in preterm infants: Indications for cochlear development? Audiology 1996; 35:296-306

AWD Gavilanes, E Heineman, M Herpers, CE Blanco. Use of neonatal intensive care unit as a safe place for neonatal surgery. Archives of Disease in Childhood 1997; 76:F1-F3 
AWD Gavilanes, E Heineman, M Herpers, CE Blanco. Use of neonatal intensive care unit as a safe place for neonatal surgery. In: AA Fanaroff, MJ Maisels and DK Stevenson: The year book of Neonatal and Perinatal Medicine, Mosby, St. Louis, MO: 351-352; 1998

F Loidl, AWD Gavilanes, E van Dijk, W Vreuls, A Blokland, JSH Vles, HW Steinbusch, CE Blanco. Effects of hypothermia and gender on survival and longterm behaviour after perinatal asphyxia in rats. Physiol Behav. $2000 ; 68(3): 263-9$.

Bruinsma, EA Stobberingh, JHM Herpers, JSH Vles, AWD Gavilanes. Subcutaneous ventricular catheter reservoir and ventriculoperitoneal drain related infections in preterm infants and young children. European Journal of Clinical Microbiology and Infection 2000; 6:202-206.

AWD Gavilanes. Consequences of perinatal asphyxia: animal experimentation and potential therapies. Ned Tijdschr Perinatale Geneeskunde 2000; 1:13-16

GH Stevens, BC Schoot, MJ Smets, B Kremer, JJ Manni, AWD Gavilanes, JT Wilmink, LW van Heurn, THM Hasaart. The ex utero intrapartum treatment (EXIT) procedure in fetal neck masses: a case report and review of the literature. Eur $\mathrm{J}$ Obstet Gynecol Reprod Biol. 2002; 100(2): 246-5

AJA de Louw, WDJ van de Berg, J de Vente, CE Blanco, AWD Gavilanes, HPJ Steinbusch, HWM Steinbusch, J Troost, JSH Vles. Developmental apoptosis in the spinal cord white matter in neonatal rats. Glia. 2002;37(1):89-91

LS de Vries, KD Liem, K van Dijk, BJ Smit, L Sie, KJ Rademaker, AWD Gavilanes. Early versus late treatment of posthaemorrhagic ventricular dilatation: Results of a retrospective study from five neonatal intensive care units in The Netherlands. Acta Paediatr 2002; 91(2): 212-217.

AJA de Louw, J de Vente, HPJ Steinbusch, AWD Gavilanes, HWM Steinbusch, CE Blanco, J Troost, JSH Vles. Apoptosis in the rat spinal cord during postnatal development; the effect of perinatal asphyxia on programmed cell death. Neuroscience 2002; 112 (4): 751-1007.

SGF Robben, JWCM Heijnens, AWD Gavilanes. Diagnose in beeld: Meconium ileus bij patiënt met cystic fibrosis. Ned Tijdschr Geneeskd 2002 28;146(52). 
